\title{
Abstracts from the 10th International Congress on Twin Studies
}

\author{
Programme Committee: Elizabeth Bryan, Isaac Blickstein, Nick Martin, Pat Preedy, Tim Spector \\ Editor: Katherine Kirk
}

\section{F ESTABLISHMENT OF A NEW POPULATION-BASED TWIN REGISTRY IN WASHINGTON STATE}

Niloofar Afari, Cynthia L. Evanoff, Dedra S. Buchwald,jack Goldberg Correspondence address: University of Washington, Harborview Medical Center Box 359780, 325 9th Avenue, Seattle, WA 98104, USA

Objectives. To establish a population-based twin registry in Washington state (USA) using driver's license applications.

Method. Data have been obtained from the Washington State Department of Licensing to identify twins. Washington asks all new drivers license applicants if they are a member of a twin pair. This question was included because twin pairs were being issued the same drivers license number when the system was implemented more than 20 years ago. However, computerized records of who is a twin pair are only maintained for 90 days before being archived to microfilm. A prospective and retrospective registry of twins is being identified.

Results. Over the past 2 years, the investigators have obtained full access to driver's license records. Close to 10,000 individual twins have been identified from prospective data (approximately 100 new twins are added per week); half are female, $42 \%$ are 20 years old or younger and $17 \%$ are born abroad, representing 103 countries. Based on a systematic random sample of microfilm reels we estimate that there are $\sim 80,000$ individual twins in the retrospective data who received a license in Washington state since 1980. More than $70 \%(\sim 56,000)$ of the twins have active drivers licenses in Washington with current name and address information.

Conclusions. These results indicate that it is feasible to construct a large twin registry in Washington. Field work is currently underway to contact a sample of twin pairs and obtain zygosity and health data.

\section{P}

\section{GENETIC \& ENVIRONMENTAL EFFECTS ON THE} INTER-RELATIONSHIP OF LYMPHOCYTE SUBSET LEVELS

Ahmadi KA, Hall MA, Norman P,Vaughan R, Snieder H, Spector TD \& LanchburyJS

Correspondence address: Twin Research \& Genetic Epidemiology Unit, St Thomas'Hospital, London, UK.

Host genetic factors play a significant role in determining resistance and susceptibility to immune related diseases. We have previously shown that genetic factors account for $50-70 \%$ of the variation in absolute numbers of CD4+ and CD8+ T and CD19+ B-lymphocytes, 3 major effector populations of the adaptive arm of the immune system. Here we used multivariate genetic modeling procedures to test whether: (a) pleiotropic genetic and environmental factors have independent effects on the co-variation of CD4+, CD8+ and CD19+ lymphocytes, or, (b) both genes and environment contribute to the variation of an unmeasured intermediate variable, which in turn controls the variation in these $\mathrm{T}$ and B lymphocyte subpopulations. Blood samples from 84 identical and 181 fraternal adult female caucasoid twins, ascertained from the St Thomas' UK twin registry, were analysed cytofluorographically. An "independent pathway" and a "common pathway" model were used in the genetic analysis of the phenotypic data. The results provide evidence for the presence of an unmeasured phenotype, which contributes significantly to the co-variation of CD4+, CD8+ T, and CD19+ B-lymphocytes. This unidentified phenotype was shown to be highly heritable $\left(\mathrm{h}^{2}=42 \%\right)$ and accounted for $58 \%, 30 \%$ and $37 \%$ of the total variance in CD4+, CD8+ T and B lymphocytes, respectively. Studies of this type facilitate the design of further laboratory experiments aimed at clarifying the biology underlying complex systems such as immunity.

\section{F TWINS AT PUBERTY - A FOLLOW-UP STUDY OF 32 TWIN PAIRS}

\section{Britta Alin Åkerman}

Correspondence address: Stockholm Institute of Education, Department of Human Development, Learning and Special Education, Box 47 308, S-100 74 Stockholm, Sweden

This is a follow up study of twins within the Stockholm area, including 32 families and their twins attending grade nine. The twins have been followed from birth onwards up to 16 years of age. The main purpose of this study is to assess the mental and cognitive development at different ages, and relate these to the teachers' ratings of the twins' adaptation to the school situation. Another aim is to see how the twins who were born prematurely are developing during the school ages. As described in earlier reports, the twins are more susceptible to lower birth weight, shorter gestation periods and birth complications. A third aim has been to gain a deeper insight into the relationship within twin-pairs and the development of their identities. Different ability tests have been used, as well as questionnaires about interests, attitudes toward school, and leisure activities. A psychological writing test including small figurers for the twins to continue has been used to examine another part of their relations to each other. In summary, it is harder for twins compared to singletons to develop independence and a positive identity, as they have to emancipate themselves both from their parents and their co-twin. Prematurity and low birth weight continued to have some relation to cognitive development from birth onwards.

\section{$004 \mathrm{P}$ ACTIVITIES IN THE LAST 3 YEARS OF JATM}

Yukiko Amau (The Japanese Association of Twins' Mothers)

Correspondence address: 5-5-20 Minami Aoyama, Minatoku, Tokyo, Japan 107

JATM was started in 1968 with only 23 mothers of twins. Now we have 3000 members all around Japan. In 1999, we petitioned the Ministry of Health and Welfare to help our activity on administrative basis.

Finally the booklet "Bringing up Twins" has been published and distributed nationwide to local health centers in 2000. Senior Supporting Team in JATM was set up in 1999 to help me. It has 20 supporters and they started to study and train with me. They participated with in the meetings of branches and gave a lot of advices for young parents. We sent our second newsletter written in English last December to 20 clubs in foreign countries for the first time in 3 years. I would like to publish it every 3 years. It has been 7 years since we have started "Twin Hot Line" phone service in April 1994. Nowadays, 41club members take part in "Twin Hot Line". And 11members are waiting for clients' calls in turn once a week in the main office. We sometimes have some training sessions to improve ourselves.

\section{$005 \mathrm{~F}$}

\section{PARENTING MULTIPLE BIRTHS:} A GROUNDED THEORY

\section{Arnette Anderson, Beverly Anderson}

Correspondence address: Faculty of Nursing, University of Alberta, Edmonton, Alberta, CanadaT6G2G3.arnette.anderson@ualberta.ca

For Canadian children and youth, fathers appear to hold a lower position of influence and importance than mothers in children's lives (Vanier Institute of the Family, 1994). As yet there is little research that gives guidance for health professionals to assist parents of multiple births in promoting father-infant(s) relationships. We have developed a comprehensive theory that explains the development of the mother-twin relationship during the infant's first year of life (MCN, 1990). Another way to broaden the current understanding of the parent-infant relationship is to focus on fathers because researchers have primarily focused on the mother-infant relationship. Furthermore, there is little research which gives guidance to health care professionals to assist fathers in developing a relationship with twin/triplets in their first year of life. This research presentation will discuss the findings of the development of the fathertwin-triplet relationship during infancy. A grounded theory approach using semi-structured interviews with 13 families at three time periods 
elicited fathers' perceptions of their father-infant(s) relationships, their fathering selves, and supportive and non-supportive environment In addition, mothers were interviewed at three time periods to bring forth their perceptions of fathering. The 76 interviews were analysed using the constant comparative method. For this presentation, the model "learning to parent multiples" will be discussed.

\section{P THE MID-ATLANTIC TWIN REGISTRY}

\section{Lisa S.Anderson, E. Lennon Murrelle, Linda A. Corey}

Correspondence address: Virginia Commonwealth University, Department of Human Genetics, Virginia Institute for Psychiatric and Behavioral Genetics, P.O. Box 980003, Richmond, VA 23298-0003, USA

The Mid-Atlantic Twin Registry (MATR), with approximately 75,000 registered twins, is one of the largest population-based twin registries in the United States. By accessing records of multiple births in Virginia, North Carolina, and South Carolina, the MATR has ascertained birth data on some 300,000 individual twins born between 1915 and the present. Juvenile twin pairs are also ascertained on a periodic basis through public and private school systems in the three states. Births in the U.S. are not routinely assigned personal identification numbers that can be used in later tracking, so the MATR has had to devise strategies to locate potential registry participants. Since one of its primary purposes is to serve as a resource for medical and behavioral research, the MATR periodically collects cross-sectional health data from adult twins. Longitudinal data on health and development of preschool-aged twins have also been collected beginning with the 1987 birth cohort. Longitudinal study of the emotional development of infant and preschoo twins will begin with the 1999 birth cohort. The MATR has served as a subject ascertainment and recruitment source for research studies on epilepsy, cancer, cardiovascular disease, anxiety and depression, alcohol disorders, adolescent and young adult behavioral development, and substance use. Upcoming project topics include polycystic ovary syndrome; the genetic basis of rheumatoid arthritis; detecting susceptibility loci for major depression; sweet preference and alcohol use; and the genetic epidemiology of high reactivity.

\section{S GENETIC STRUCTURE OF WORKING MEMORY AND ITS RELATIONSHIP TO BRAIN FUNCTION}

Juko Ando, Kaname Mochizuki, Kai Hiraishi, Hiroshi Maruyama, Hiroko Maekawa, Naoko Onoda, and Yutaka Ono

Correspondence address: Keio University,Tokyo,108-8345, Japan; juko@msa.biglobe.ne.jp

Working memory (WM) is defined as active maintenance (storage) and executive functions of short-term memory. In this study, genetic structure of these two different functions (storage and executive) in two different modalities (spatial and verbal) was investigated by the twin method. One hundred and forty-two pairs of monozygotic and 92 paris of dizygotic Japanese twins took spatial and verbal working memory tasks, and $89 \mathrm{MZ}$ and $65 \mathrm{DZ}$ twins among them took the higher-order spatial and verbal cognitive tests. Spatial storage (Ss), spatial executive (Se), Verbal storage (Vs), and verbal executive (Ve) were measured as WM parameters, and spatial cognitive ability (SC) and verbal cognitive ability (VC) scores were constructed from the standardized Japanes Intelligence test (Kyodai Nx). Univariate genetic analysis showed that all WM parameters and higher-order cognitive ability scores were affected by additive genetic and nonshared environmental effects. Multivariate genetic analysis indicated that there is one common genetic factor with modality-specific genetic factors underlying WM parameters. The results also suggest that WM parameters were genetically related to higher-order cognitive abilities. Preliminary finding on the relationship between WM and Event Related Potential (ERP) components (P300 and Slow Wave) in the Delayed Response Task will be discussed.

\section{F SELECTIVE TERMINATION IN TWIN PREGNANCIES DISCORDANT FOR HOMOZYGOUS BETA THALASSAEMIA}

Aris Antsaklis, George Daskalakis,Afroditi Loutradi, Katerina Sinopoulou, Spyros Mesogitis, Stelios Kitmirides, Panayiota Bitziou

Correspondence address: 1st Department of Obstetrics and Gynaecology,

University of Athens, 80 Vas. Sophias Street, 11528 Athens, Greece

Objective. To study the perinatal outcome of twin pregnancies discordant for homozygous beta thalassaemia, following selective termination of the affected twin.

Methods. We conducted a retrospective study of 70 cases of twin pregnancies discordant for homozygous beta thalassaemia.

Results. 28 of the terminations were performed during the 1st trimester The median gestational age at termination was 11.4 weeks. 2 women miscarried $(7.1 \%)$, while the median gestational age at delivery was 36.6 weeks. 4 women delivered before 34 weeks $(14.2 \%)$ and 3 out of them $(10.7 \%)$ delivered before 30 weeks. There were also 52 terminations in the 2nd trimester. The median gestational age at termination was 18.6 weeks. 2 of our patients miscarried $(3.9 \%)$. The median gestational age at delivery was 35.9 weeks. 14 patients $(28 \%)$ delivered before 34 weeks, while 6 out of them $(12 \%)$ delivered before 30 weeks

Conclusion. Selective termination in twin pregnancies discordant for homozygous beta thalassaemia is associated with a favorable outcome of the unaffected twin. However, the preterm delivery rate before 34 and 30 weeks' gestation is high, when the termination is performed in the second trimester.

\section{P AMNIOCENTESIS IN NATURAL AND IVF TWIN PREGNANCIES}

Aris Antsaklis, George Daskalakis, Dimitrios Loutradis, Konstantinos Kallianidis, Nikolaos Papantoniou, Daniela Paouleskou, Evangelia Doula

Correspondence address: 1st Department of Obstetrics and Gynaecology:

University of Athens, 80 Vas. Sophias Street, 11528 Athens, Greece

Objective. The aim of our study was to investigate if amniocentesis carries a higher risk in twin pregnancies conceived after in vitro fertilization (IVF), than in naturally conceived twins.

Methods. The study group consisted of 308 normally conceived twin pregnancies and of 145 twin pregnancies conceived following IVF. Amniocentesis was performed between 16-19 weeks' gestation. Pregnancies which had undergone multifetal pregnancy reduction were excluded from the study. The main outcome variables were pregnancy loss and preterm delivery rate.

Results. The median maternal age was not significantly different between the two groups (35.9 vs 36.4 years in naturally and IVF conceived pregnancies). Pregnancy loss occurred in $3.6 \%$ of naturally conceived pregnancies and in $4.1 \%$ of IVF ones. Preterm delivery rate $(<32$ weeks) was 6.4 and $14.4 \%$ in the two groups, respectively, while that of very early preterm deliveries ( $<28$ weeks) was 6.5 and $0.7 \%$, respectively.

Conclusions. Twin pregnancies resulting after IVF are not at a higher risk of pregnancy loss following amniocentesis, compared to normally conceived twins. However the risk of early preterm delivery $(<32$ weeks $)$, as well as that of very early preterm delivery $(<28$ weeks $)$ is significantly higher.

\section{F DISTINGUISHING CAUSAL FROM NON-CAUSAL ASSOCIATIONS USING RANDOM EFFECTS REGRESSION MODELS FOR TWIN DATA}

Lester M Arguelles, Seth Eisen,William R True, Michael Lyons, Ming T Tsuang, Jack Goldberg

Correspondence address: VET Registry, Hines VA Hospital, IL, 60141 USA

Objectives. To use random effect regression models to examine the association of service in Vietnam with post-traumatic stress disorder (PTSD) and alcoholism.

Methods. Data were derived from psychiatric interviews conducted with $\sim 3,000$ twin pairs in the Vietnam Era Twin Registry. Following Kendler et al. (Arch Gen Psych 1993, 50, 36-43) three alternative random effect models were fit to the data to distinguish causal (environmental) from non-causal (genetic) hypotheses: model 1 tests the general association between Vietnam service and the outcomes; model 2 examines if the model fit is improved by including separate parameters for between and within twin pair effects, and; model 3 examines if the association is different within DZ and MZ twins.

Results. When a single effect parameter is estimated there is a significant association of Vietnam service with both PTSD $(\mathrm{OR}=3.2,95 \%$ CI 2.6-3.9) and alcoholism (OR $=1.3,95 \%$ CI 1.2-1.5). For PTSD, separate parameters for within and between effects and DZ versus MZ fail to alter the basic 3-fold association. For alcoholism the association with Vietnam service is gradually diminished in the sequential models. Between effects $(O R=1.4)$ are larger than within effects $(O R=1.3)$ and within $\mathrm{DZ}$ effects $(\mathrm{OR}=1.5)$ are larger than within $\mathrm{MZ}$ effects $(\mathrm{OR}=1.1)$.

Conclusions. Using random effects regression models these analyses suggest that PTSD is causally linked to service in Vietnam while the Vietnam-alcoholism association is a non-causal association due to confounding by shared genetic effects.

\section{F TWIN INFANT SLEEPING ARRANGEMENTS AND IMPLICATIONS FOR SIDS}

Helen L. Ball, Emma Kitching, Elaine Hooker, Joanne Anderson-Hawkins Correspondence address: Department of Anthropology, University of Durham, 43 Old Elvet, Durham, DH1 3HN

There is a $42 \%$ increased risk of SIDS for twin infants compared to singletons, and the greatest reduction in singleton cot-deaths has occurred via interventions in infant care practices. Current advice to parents regarding 
reducing the risk of SIDS recommends that babies should sleep in a supine position with their feet to the foot of the crib. The 'feet-to-foot' advice was designed to minimise the risk of an infant slipping under the bedcovers, and subsequently overheating or suffocating, and is geared towards singleton infants sleeping alone. Parents of many twins (due to preference or circumstance) 'co-bed' their infants but little advice exists about the safest way to do so. This paper reports on a study designed to explore the sleeping arrangements and night-time care-giving strategies employed by parents with twin infants. We use qualitative and quantitative data generated via sleep logs compiled for a seven-day period in each of the infants' first month and third month, combined with semi-structured interviews regarding sleeping strategies conducted at the end of the first and third months, to explore the various sleeping arrangements and their permutations employed by parents for their twin infants. Parents who co-bed from choice cite better infant sleep, and thus more parental sleep, as a motivating factor, however there are concerns regarding compression of one infant by another when placed side-by-side, or entanglement/overheating in each other's covers when placed end-to-end. Some parents devise barriers (cushions, rolled up towels) to separate their infants, but these might introduce hazards of their own in to the sleep environment. The pros and cons of twin infant cobedding are explored.

\section{F PARENTING STRESS AMONG PARENTS OF TWINS: IVF VS. SPONTANEOUS}

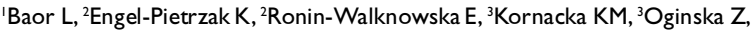
${ }^{3}$ Bros M, ${ }^{4}$ Blickstein I.

Correspondence address: Universities of Tel Aviv $(I L)^{l}$, Szczecin $(P L)^{2}$, Warsaw $(P L)^{3}$ and Jerusalem(IL) baor@post.tau.ac.il

Parenting stress was assessed by direct questioning of 56 Polish mother of twins delivered within the last 24 months $(9 \mathrm{mo}+6$, range: $1-23)$ Mothers were $30+5$ yrs old (range: 29-40), mostly nulliparous, $28.6 \%$ conceived by ART, had $13.2+4$ years of education, and mainly $(94.7 \%)$ of average low to average high economic status. GA at birth was 35.6 +2.4 wks (range 28-40), BW of twin A was $2442 \mathrm{~g}+602$ (range: $1100-3820$ ) and of BW of twin B was $2420 \mathrm{~g}+648$ (range: 1020-4160). NICU admission was 20/56 and 24/56 for twin A and B, respectively. The short form Parenting Stress Index (Abidin RR) was calculated for each mother. A score of $>90$ was considered significantly severe. We found that 8/15 ART and 10/39 spontaneous conceptions had a score $>90(p=.11)$. Comparison of individual twin BW, economic status, level of education, gestational age, and twin age at time of assessment were not different. We conclude that in this sample of Polish mothers, severe parenting stress was found in half of the mothers following ART conceptions, twice as much as in the spontaneous group. Parenting stress in this group seems to be uninfluenced by mode of conception as well as the other tested variables.

\section{P TYPE A BEHAVIOR IN MALE TWIN PAIRS: THE VIETNAM ERA TWIN REGISTRY}

Jessica L. Bar, Michael J. Lyons, Hong Xian, and Ming Tsuang

Correspondence address: Boston University, 64 Cummington Street, Boston, MA 02215

We examined genetic and environmental influences on Type A behavior using data collected from members of the Vietnam Era Twin (VET) Registry. The Registry comprises male twin pairs who served in the U.S. military between 1965 and 1975. A 1990 survey mailed to the twins included items from the Framingham Type A Scale. Data was received from over $1500 \mathrm{MZ}$ and $1100 \mathrm{DZ}$ pairs. We found that a model including additive genetic influences and unique environmental influences provided a good fit to the data. Our results are similar to those of past studies which found that individual differences in Type A behavior depend more on unique environmental experiences than on genetic factors. Our results are also in accord with previous studies showing no influence of the shared familial environment on Type A behavior.

\section{P ABNORMAL AMNIOTIC FLUID VOLUME IN TWIN GESTATIONS IS ASSOCIATED WITH HIGHER RATES OF PERIPARTUM COMPLICATIONS}

Jury Bar-David, Zahi Ben-Aroya, Mordechai Hallak, Michael Friger, Miriam Katz and Moshe Mazor

Correspondence address: Dept. of Obstetrics \& Gynaecology, Soroka University Medical Center, Beer-Sheva, ISRAEL

Objective. Assess the effects of poly and oligohydramnion in twin gestation on rates of peripartum complications.

Study design. Study population were 917 women with twin gestation and leading fetus in vertex presentation. 47 women had polyhydramnion $(\mathrm{AFI}>24)$ and 22 women had oligohydramnion $(\mathrm{AFI}<5)$. The remaining 848 served as controls. We compared the rates of preterm births, 5 minutes Apgar score $<7$, labour dystocia, fetal distress, abruptio placenta, post partum hemorrhage, vacuum deliveries and cesarean sections
(CS) rates in the three groups with ANOVA, $\chi^{2}$ analysis and Fisher's exact test when appropriate. Multivariate analysis was done to evaluate the effect of abnormal amniotic fluid volume on CS rate.

Results. Polyhydramnios was associated with shorter pregnancy duration than control group (33.8w vs 36w; $p<0.01$ ), increased rate of low Apgar score at $1 \mathrm{~min}-(20.5 \%$ vs. $4.6 \% p<0.01)$ and CS $(48.9 \%$ vs. $31.6 \%$ $p=0.02$ ). Olygohydramnios was associated with increased rate of CS $(72.7 \%$ vs. $31.6 \% p<0.01)$. No significant changes were found in the rates of other peripartum complications. Multivariate analysis revealed that poly and olygohydramnion are independent risk factors for CS in twin gestation $(\mathrm{RR}=2.28 ; p=0.01$ and $\mathrm{RR}=6.75 ; p=0.002)$

Conclusions. Polyh and olygohydramnion are risk factors for CS in twins. Polyhydramnion is also associated with higher rates of preterm births and low Apgar scores at 1 minute.

\section{S THE BIOLOGICAL AND SOCIAL ORIGINS OF CORONARY HEART DISEASE}

DJP Barker

Correspondence address: MRC Environmental Epidemiology Unit, Southampton General Hospital, Southampton SO16 6YD

Epidemiological studies provide a substantial body of evidence that people who had low birthweight, or who were thin or short at birth, or who failed to grow in infancy, have increased rates of coronary heart disease, stroke, non-insulin dependent diabetes and hypertension. This has led to the fetal origins hypothesis, which proposes that these diseases originate through adaptations which the fetus and infant make when they are undernourished. These adaptations include diversion of oxygenated blood away from the trunk to favour the brain, alterations in the hormonal systems which regulate growth and maturation, importantly insulin and cortisol, and alterations in body composition. The adaptations permanently change the structure and function of the body. The path of growth through childhood modifies the risk of disease associated with small body size at birth. The highest death rates from coronary heart disease among men occur in those who were thin at birth and at one year but whose weight gain accelerated in childhood so that they had an above average body mass index. Death from coronary heart disease may therefore be a consequence of poor prenatal or infant nutrition followed by improved postnatal nutrition. Other patterns of fetal and childhood growth are associated with the later development of stroke, non-insulin dependent diabetes and hypertension and the patterns differ between the two sexes. Common to them all is a period of reduced early growth followed by a period of accelerated growth.

The persisting changes in the body's structure and function that are associated with reduced early growth alter the body's responses to adverse biological and social influences in later life. For example, people who were small at birth are more prone to developing Type 2 diabetes or coronary heart disease if they become overweight in adult life. A number of maternal influences which programme the fetus have now been identified. They include the mother's body composition before and during pregnancy and her diet in pregnancy. A new public health agenda for the prevention of chronic disease is emerging. Simple calculations show that quite modest improvements in fetal and infant growth would lead to substantial falls in disease rates.

\section{S MULTIPLE EXPOSURE: AN INNOVATIVE APPROACH TO THE ANTENATAL CARE OF MULTIPLE PREGNANCY}

Jon Barrett

Correspondence address: Sunnybrook \& Women's College Health Sciences Centre, University of Toronto, Ontario M5S 1 B6 Canada

Based on a multidisciplinary model of care, the Antenatal Care of Multiple Pregnancy Centre (ANCOMP) was established to meet the complex needs and special concerns of women and families experiencing a multiple pregnancy. ANCOMP offers a multidiscipli nary standard of care that will best meet the obstetrical and psychosocial needs of the woman and her family. Women have access to expertise from perinatologists, neonatologi sts, perinatal ultrasonographers, dieticians, social workers and parents of multiples. Along with the Clinical Nurse Specialist, each woman identifies her educational and support needs and referrals are made appropriately. Close links with community support groups such as Multiple Births Canada are maintained and all families are encouraged to attend prenatal classes specifically for parents expecting multiples. 


\section{S A TWIN STUDY OF LOW BACK SYMPTOMS}

Michele C. Battié, Jaakko Kaprio, Tapio Videman, Esko Levälahti. Correspondence address: Faculty of Rehabilitation Medicine, University of Alberta, Edmonton, Canada T6G $2 G 4$

Background. Low back pain problems are among the most common and costly musculoskeletal problems facing the developed countries of the world. Yet, little is known about their etiology or underlying pathology. Recent evidence suggests that disc degeneration and failure, commonly suspected as underlying culprits, are substantially influenced by genetic factors. To quantify the genetic effects on back symptoms we conducted a twin study.

Methodology. Detailed interviews of low back pain history and exposure to suspected environmental risk factors were conducted of $147 \mathrm{MZ}$ and $153 \mathrm{DZ}$ male twin pairs, 35 to 69 years of age, drawn from the population-based Finnish Twin Cohort. Different back symptoms were assessed for genetic vs. environmental effects through standard modelfitting techniques in the structural equation model-fitting program $\mathrm{Mx}$.

Results. For current back pain, additive genetic effects (AE model, AIC $=-7.55)$ accounted for $37 \%(95 \%$ CI $10-61 \%)$ of variance in liability, while for frequency of back pain in the past 12 months, genetic effects accounted for $30 \%(13-36 \%)$ of phenotypic variance. The best model for lifetime back pain lasting more than one day was a CE model with $21 \%(0-43 \%)$ of liability accounted for by shared environmental effects. The largest genetic component was observed for average disability score $(41 \%, 95 \%$ CI $24-57 \%)$, but for most other measures the genetic component was modest $(<30 \%$ in an AE model).

Conclusion. Among middle aged male twin pairs, genetic effects on various measures of back pain appear to be moderate.

\section{S TWIN STUDIES IN METABOLIC DISEASE: TYPE 2 DIABETES AND THE METABOLIC SYNDROME}

Henning Beck-Nielsen, Pernille Poulsen,Allan Vaag, Kirsten Kyvik.

Correspondence address: Endocrinological Department,

Odense University Hospital, Denmark.

Results obtained by using the Danish Twin Register which is population based, in studies of the pathophysiology to type 2 diabetes and it's ethiological determinence: insulin resistance and betacell function will be discussed. Together with that, the genetic influence on the variation in the components of the metabolic syndrome will be discussed.

Major findings. (a) The genetic component in development of type 2 diabetes is lower than previously published; (b) only about $25 \%$ of the variation in insulin resistance is genetically determined; (c) about 50\% of the variation in betacell function is genetically determined; (d) the components in the metabolic syndrome is determined by both genetic and environmental factors. The gender seems to be a very important variable in studies of genetic traits which has not been taken into consideration in most "gene hunting" studies. Furthermore, it will be discussed how intrauterine nutrition can influenze the results obtained in twin studies. In conclusion, twin studies together with the candidate gene approach clearly indicate that investigations of the pathophysiology of type 2 diabetes and the metabolic syndrome should focus more on environmental factors.

\section{F THE UNIVERSAL DILEMMA: THE EDUCATIONAL NEEDS OF TWINS}

Patti Beemer and KellyWillenberg

Correspondence address: P.O. Box 438, Thompson Station, Tennessee, 37179-0438, USA

Whether to place twins/triplets together or apart in the classroom continues to be one of the greatest challenges facing multiple birth families and educators. This session examines educator assumptions concerning multiples and classroom placement, reviews the current research on this topic and provides ways all involved parties can better meet the needs of this growing population of students. After extensive research with multiple birth families and educators at the pre-school through high schoo level, the National Organization of Mothers of Twins Clubs, Inc. created our Multiple Birth Education Kit. These unique resources offer innovative materials crucial to providing quality education and making equitable decisions for multiple birth children and their families. This presentation will review a variety of issues surrounding placement decisions. In addition, NOMOTC has developed unique interactive materials for use with educators and other support personnel to offer hands-on experience and solutions to the most common dilemmas faced with multiples in school. By accessing the latest research and these original techniques, educators and parents will be able to network together to enhance the education of twins and higher order multiple birth children. NOMOTC offers new insights and perspectives on this universal dilemma. IS IT REALLY A PROBLEM?

Zahi Ben-Aroya,Jury Bar-David, Mordechai Hallak, Michael Friger Miriam Katz and Moshe Mazor

Correspondence address: Dept. of Obstetrics \& Gynaecology, Soroka University Medical Center, Beer-Sheva, ISRAEL.

Objective. To determine whether grandmultiparity in women with twin gestation exposes the parturient to higher rates of peripartum complications.

Study design. Between the years 1989-1998, 917 partuerients with twin gestation delivered in our institution. The study group consisted of the 161 grandmultiparous women (parity $>5$ ). The control group consisted of the remaining 756 women with parity $<6$. We compared the rates of placental abruption, meconium stained amniotic fluid, Apgar scores, fetal distress, intrapartum fetal death (IPFD), post partum hemorrhage $(\mathrm{PPH})$, uterine rupture, birth canal lacerations, vacuum deliveries and cesarean sections rate in these two groups by using $\chi^{2}$ analysis and Fisher's exact test if needed.

Results. No significant increase in the rates of uterine rupture $(0 \%$ vs. $0 \%$ ), meconium stained amniotic fluid (7.5\% vs. $6.3 \%)$, placental abruption $(2.5 \%$ vs. $1.6 \%)$, IPFD ( $1.2 \%$ vs. $0.3 \%)$, PPH (1.2\% vs. $0.7 \%)$, birth canal lacerations $(1.2 \%$ vs. $0.5 \%)$, fetal distress ( $4.3 \%$ vs. $7.3 \%)$, Apgar scores of less than 7 after 5 minutes $(1.3 \%$ vs. $0.3 \%)$, vacuum deliveries $(2.5 \%$ vs. $3.4 \%)$ and cesarean sections rate $(31.1 \%$ vs. $34 \%)$ were shown between the study and the control groups, respectively.

Conclusions. Grandmultiparity in twin gestation was not associated with increased risk of peripartum complications. Therefore grandmultiparity should not be considered a significant risk factor in twin pregnancies when modern facilities are available.

\section{RIPENING OF UTERINE CERVIX IN PARTURIENTS WITH TWIN GESTATION: $\mathrm{PGE}_{\text {, }}$ v. FOLEY CATHETER}

Zahi Ben-Aroya, Jury Bar-David, Mordechai Hallak, Michael Friger, Miriam Katz and Moshe Mazor

Correspondence address: Dept. of Obstetrics \& Gynaecology, Soroka University Medical Center, Beer-Sheva, ISRAEL.

Objective. To compare the success and complication rates of prostaglandin- $\mathrm{E}_{2}\left(\mathrm{PGE}_{2}\right)$ and Foley catheter for the ripening of uterine cervix in partuerients with twin gestation.

Study design. Study population consisted of partuerients with twin gestation and leading fetus in vertex presentation that underwent ripening of uterine cervix using $\mathrm{PGE}_{2}(n=17)$ or Foley catheter $(n=24)$ as part of the labor induction process. Controls were 870 women with no induction of labor. We compared the rates of placental abruption, fetal distress, intrapartum fetal deaths (IPFD), uterine rupture, Apgar scores, labor dystocia, birth canal lacerations, vacuum deliveries and $\mathrm{CS}$ rates in the three groups using ANOVA, $\chi^{2}$ analysis and Fisher's exact test when appropriate.

Results. A significant increase in the rate of labor dystocia during 1st stage was observed in the Foley group as compare to controls $(12.5 \%$ vs. $2 \% p=0.01)$. No such changes were shown in the PGE group as compared to the control group. A higher rate of CS was noted in the Foley group as compared to the $\mathrm{PGE}_{2}$ group $(45.8 \%$ vs. $11.8 \% p=0.04)$. No differences were found between the PGE group and Foley group as compared to the control group in the rates of other modalities checked. Conclusions. Both $\mathrm{PGE}_{2}$ and Foley catheter were safe for the ripening of uterine cervix in partuerients with twin gestation. $\mathrm{PGE}_{2}$ was superior to Foley catheter as less labor dystocias and cesarean sections were demonstrated.

\section{P THE USE OF OXYTOCIN FOR AUGMENTATION OF LABOR IN TWIN PREGNANCIES: IS IT SAFE?}

Zahi Ben-Aroya,Jury Bar-David, Mordechai Hallak, Michael Friger, Miriam Katz and Moshe Mazor.

Correspondence address: Dept. of Obstetrics \& Gynaecology, Soroka University Medical Center, Beer-Sheva, ISRAEL

Objective. Determine whether the use of oxytocin for the augmentation of labor in women with twin gestation increases the risk of peripartum complications.

Study design. Among the 917 women with twin gestation who delivered in our institution, 60 had intravenous oxytocin for augmentation of labor. The control group were the remaining 857 partuerients. We compared the rates of placental abruption, meconium stained amniotic fluid, Apgar scores, fetal distress, intrapartum fetal death (IPFD), post partum hemorrhage (PPH), uterine rupture, birth canal lacerations, vacuum deliveries and cesarean section rate in these two groups by using $\chi^{2}$ analysis and Fisher's exact test when appropriate. 
Results. No significant increase in the rates of uterine rupture $(0 \%$ vs. $0 \%)$, meconium stained amniotic fluid $(6.7 \%$ vs. $5 \%)$, placental abruption (0\% vs. $1.9 \%)$, IPFD ( $0 \%$ vs. $0 \%)$, PPH $(0 \%$ vs. $0.8 \%)$, birth canal lacerations $(1.6 \%$ vs. $0.9 \%)$, fetal distress $(6.7 \%$ vs. $6.8 \%)$, Apgar scores of less than 7 after 5 minutes $(0 \%$ vs. $0.5 \%)$, vacuum deliveries $(6.7 \%$ vs $3 \%)$ and cesarean section rate $(23.3 \%$ vs. $34.2 \%)$ were demonstrated between the oxytocin augmented and the control groups, respectively.

Conclusions. The use of oxytocin for the augmentation of labor in partuerients with twin gestation was found to be relatively safe with no significant increase in peripartum complications.

\section{F RISK FACTORS FOR CESAREAN SECTION IN TWIN GESTATIONS}

Zahi Ben-Aroya, Jury Bar-David, Mordechai Hallak, Michael Friger, Fernanda Press, Miriam Katz and Moshe Mazor

Correspondence address: Dept. of Obstetrics \& Gynaecology, Soroka University Medical Center, Beer-Sheva, ISRAEL

Objective. To determine the independent risk factors for cesarean section (CS) for partuerients with twin gestation.

Study design. Between the years 1989-1998, 917 partuerients with twin gestation, with leading fetus in vertex presentation, delivered in our institution. We assessed the effect of maternal age, parity, gestational age, fetal weight, ripening of uterine cervix using Foley catheter or PGE, amniotomy, preterm rupture of membranes, oxytocin augmentation of labor, epidural analgesia, pregnancy induced (PIH) and chronic hypertension, poly or oligohydramnion, gestational diabetes (GDM), epidural analgesia and meconium stained amniotic fluid on CS rate. Multivariate analysis was performed to evaluate the factors mostly and independently affecting the need for CS.

Results. The factors found to significantly affect CS rate are shown in the Table below.

Conclusions. Oligo and polyhydramnion, epidural analgesia, GDM and $\mathrm{PIH}$ are all independent risk factors for CS in twin gestations.

\section{Risk factors for CS}

\begin{tabular}{lcc}
\hline Factor & Relative Risk & $\mathrm{Pv}$ \\
Oligohydramnion & 6.75 & 0.002 \\
Epidural analgesia & 5.36 & 0.001 \\
Gestational Diabetes & 3.78 & 0.008 \\
PIH & 2.89 & 0.001 \\
Polyhydramnion & 2.28 & 0.018 \\
\hline
\end{tabular}

\section{F CYCLO-OXYGENASE EXPRESSION IN IDENTICAL} TWINS DISCORDANT FOR TYPE 1

H Beyan, RDG Leslie, M. R. Goodier, N Sirwan-Muhamad, L Buckley, MI Hawa, P Sawtell, S Bustin, N Yousaf, M. Londei.

Correspondence address: Department of Diabetes, St Bartholomew's Hospital, UK

Cyclo-oxygenas e-2 (COX-2) is responsible for the synthesis of prostaglandin in mammalian cells. A recent report suggests an aberran monocyte COX-2 expression in type 1 diabetes, and those at risk of the disease. Aberrant monocyte COX-2 expression may result in antigen presenting cell dysfunction, thereby predisposing these individuals to diabetes. To define the nature of monocyte COX-2 expression in type 1 diabetes and to determine whether increased expression could be inherited, we are studying a large cohort of identical twins discordant for type 1 diabetes. Using human peripheral blood mononuclear cells (PBMCs) we isolated $\mathrm{CD} 14^{+}$monocytes and tested for COX-2 messenger RNA using quantitative RT-PCR, intracellular COX-2 protein expression by FACS and on western blot (WB), as well as functional studies employing cytokine and prostaglandin secretion, before and after lipopolysaccharide (LPS) stimulation with and without COX-2 inhibitors. Normal healthy controls do have detectable COX-2 mRNA but protein expression was below detectable levels on FACS and on WB. In contrast, twins with type 1 diabetes have COX-2 protein expression on both FACS intracellular staining and on WB. Non-diabetic twins, at low disease risk with normal glucose tolerance and without diabetes-associated autoantibodies showed COX-2 expression in unstimulated cells intracellularly on FACS compared to normal controls $(p<0.004)$ Our study confirms that basal COX-2 expression is abnormal in type 1 diabetes. Monocytes in type 1 diabetes are activated and this activation is not entirely due to diabetes but associated with disease susceptibility. These present observations could have important implications for our understanding of the pathogenesis of diabetes.

\section{Isaac Blickstein}

Correspondence address: Isaac Blickstein, Dept of Ob/Gyn Kaplan Medical Center, Rehovot, Israel; blick@netvision.net.il

Nomansland is a small country with exactly 100,000 deliveries/year. Because of religious and political reasons, fertility treatment was prohibited. By 1.1.90, a new regime changed the rules, introduced ART as the only treatment, but for economic grounds - ART was limited to exactly 10,000 births/year. ART results and perinatal outcome were exactly as in the USA (Alexander et al., 1998)

\begin{tabular}{lccc} 
& Before ART & With ART & Change \\
\hline Births & 100,000 & 10,000 & $+10 \%$ \\
Total & 98,900 & 7,000 & $+7.1 \%$ \\
Singletons & 1,000 & 2,500 & $+250 \%$ \\
Twins & 100 & 500 & $+500 \%$ \\
Triplets & 101,200 & 13,500 & $+13.3 \%$ \\
Neonates & 2084 & 1434 & $+69 \%$ \\
$<33$ weeks & 7312 & 4409 & $+60.3 \%$ \\
$<2500 \mathrm{~g}$ & 1386 & 1061 & $+76.5 \%$ \\
$<1500 \mathrm{~g}$ & 176 & 105 & $+60 \%$ \\
CP cases & & & \\
\hline
\end{tabular}

Based on these results, the Ministry of Health of Nomansland called upon the WHO to help eradicate the epidemic.

An International Task Force revealed that quite soon this situation would prevail in most developed and in many developing countries. Therefore, no help could be offered.

\section{S THE IMPACT OF THE INCREASING NUMBER OF MULTIPLE BIRTHS ON THE INDICATORS OF PREGNANCY OUTCOME}

Béatrice Blondel, Michael D Kogan, Greg R Alexander, Michael S Kramer, Shi Wu Wen

Correspondence address: INSERM U149, 16 avenue Paul Vaillant Couturier, 94807 Villejuif cedex, France

The increasing incidence of multiple births in most developed countries substantially affects the indicators of pregnancy outcome in the overall population, as twins and triplets are at higher risk of mortality, preterm delivery, low birthweight, congenital anomalies and cerebral palsy compared with singletons. The contribution of twins and triplets to the rise or the stabilization of the overall rates of preterm delivery was assessed in Canada, France and the USA in the 1980s and 1990s using data from vital statistics and national samples of births. Because of their larger number, twins contributed much more than triplets to the numbers of preterm deliveries. For example in Canada in 1995-97, $14 \%$ of deliveries before 37 weeks were attributable to twins and $1 \%$ to triplets. Between 1981-83 and 1995-97 the proportion of preterm deliveries attributable to twins increased from $13.5 \%$ to $18.7 \%$ in France and from 6.6 to $10.3 \%$ in the USA. These results suggest that the indicators of pregnancy outome should be analysed separately for singleton s and multiples. Furthermore any medical practice which would reduce the rate of twin pregnancy or the rate of adverse pregnancy outcome among twins may have an impact on the indicators of pregnancy outcome in most developed countries.

\section{F MECHANISM OF NATURAL DIZYGOTIC TWINNING}

\section{Charles E. Boklage}

Correspondence address: East Carolina Univ School of Medicine, Greenville NC27858-4354 USA boklagec@mail.ecu.ed

It is a basic belief of twin biology that dizygotic co-twins are conceived by fertilization of independently ovulated oöcytes, and that they therefore have, as a group, the same distribution of genetic relationships as among non-twin sib-pairs. However, after 30 years studying the literatures of human embryogenesis, twinning, and genetic twin studies, I cannot tell you where to find any evidence to support this belief. We have instead steadily accumulated significant evidence that dizygotic twins differ from singletons in their development at least as much as monozygotic twins do, and generally in the same ways. Those differences are concentrated in embryogenic determination of structures dependent upon midline fusion of asymmetric half-structures, notably brain, face and heart hard to explain if embryogenesis of DZ twins must begin with independent and ordinary oöcytes. Several reports have documented significantly excessive dizygotic co-twin matching for markers near centromeres, and a high frequency of chimerism between dizygotic co-twins 
has recently come to light, extremely unlikely under an hypothesis of double ovulation. We have in progress a genome scan, with 390 markers covering the genome at an average interval of $10 \mathrm{cM}$, in 66 families of naturally conceived dizygotic twins or triplets. Here, double ovulation with ordinary sib-sib segregation is not assumed as common knowledge, but is the null hypothesis to be effectively tested for the first time against concrete physical evidence. We expect to determine directly whether genetic segregation between dizygotic co-twins is the same as that between non-twin siblings, and will report to the Congress the results of this first direct test of that standard assertion.

\section{S A STUDY OF ASTHMA AND ALLERGIES IN 5-YEAR-OLD DUTCH TWINS}

\section{D.I. Boomsma, C.E.M. van Beijsterveldt \\ Correspondence address: Dept of Biological Psychology, Vrije Universiteit, \\ Van der Boechorstraat 1, 1081BT, Amsterdam, The Netherland.}

We looked at twin resemblances for asthma and allergies in a populationbased sample of 3600 young twin pairs born in The Netherlands The parents of these twins participate in a longitudinal survey study of growth and development of their children. Around the 5th birthday of the twins, parents receive a questionnaire that contains questions about wheezing and coughing as judged by the parents in the last 12 months. Parents are also asked if a doctor ever diagnosed asthma, allergies, hayfever, eczema, bronchitis or pneumonia in their children. Results show relatively low associations between the different symptoms. For all symptoms correlations between monozygotic twins are high $(0.50-0.80)$. In dizygotic twins the correlations are usually half the MZ correlations. There are no differences in correlations between male and female twin and the correlations in opposite-sex twins are similar to those in DZ same-sex twins.

These data thus suggest:

- strong genetic influences on the liability to asthma and allergies,

- no sex differences in genetic architecture and

- no influence of shared environmental factors in 5 year-old-children.

\section{S GENETIC AND ENVIROMENTAL INFLUENCE ON SOCIAL ATTITUDES, VALUES, INTERESTS AND PERSONALITY}

Thomas J. Bouchard,Jr.

Correspondence address: Psychology Depatment, University of Minnesota, Minneapolis, MN 55455

Most human individual differences have their analogs in primates and other mammals. Thus cognitive abilities, motor skills, and even personality have been studied in lower organisms. It is more difficult to conceive of social attitudes outside the context of culture Religiousness, Conservatism, Authoritarianism appear on their face to be uniquely human traits and exclusively influenced by environmental factors - largely familial. Initially behavior geneticists claimed these measures were not influenced by genetic factors. They were misled by studies carried out on young twins. Recent behavior genetic studies have shown that social attitudes are very significantly influenced by genetic factors and this finding cannot be "explained" by nuisance variables such as personality or intelligence. Attitudes also demonstrate much higher assortative mating coefficients and different age curves than personality. These measures are also associated with health related behavior and reproductive fitness, raising the question of their possible status as "adaptive traits". The findings in this domain and their implications for future behavior genetic research will be discussed

\section{S THE BROKEN TWINSHIP}

\section{Elizabeth Bryan}

Correspondence address: The Multiple Births Foundation, Queen Charlotte's and Chelsea Hospital, Du Cane Rd, London W12 ONS, UK

The unique relationship of twins can be broken in many ways. The effects on the survivor and on the parents can be profound and are still not fully appreciated or even understood. For every pair of twins born there are many singletons conceived with a 'vanishing twin' The physical and emotional impact of this and especially of the first trimester reduction of a multifetal pregnancy needs further investigation. The perinatal mortality of twins is five fold that of singletons and of triplets is ninefold. Parental responses are complicated by simultaneous joy and grief, the enduring reminder of the dead twin in the living twin and the loss of a special status. In the surviving twin, guilt, anger, resentment and loneliness are not uncommon and survivors of a stillborn partner have a higher psychiatric morbidity. In some cultures the spirit of the twin is thought to live on in the survivor. In others, the survivor may regard themselves as half persons. Survivors of strongly interdependent pairs have described the death of their twin as an amputation.
Bereavement results not only from death but also from separate adoption in infancy or the effective loss of the twinship through distance, conflict or emotional separation (eg following a new partnership) or through disability or disfigurement. It is only by listening to parents and twins themselves that we shall unravel and understand these complex issues.

\section{S LOSS IN HIGHER MULTIPLE PREGNANCY AND MULTIFETAL REDUCTION}

\section{Elizabeth Bryan}

Correspondence address: The Multiple Births Foundation, Queen Charlotte's

and Chelsea Hospital, LondonW12 OHS. ebryan@higgins7.co.uk

From early pregnancy into childhood, higher multiples have much higher rates of mortality, whether from spontaneous abortion, the 'vanishing twin' syndrome, fetal or infant death (the perinatal mortality rate of triplets is about 9 times that of singleborns). Many parents must cope with the death of one baby whilst the siblings remain critically ill or later become disabled. Babies born after years of infertility are especially precious but the loss is often underestimated when there is a surviving child. For parents who choose a fetal reduction, whether for medical or social reasons, pain and grief are inevitable and need to be fully acknowledged. The couple needs information* and support both while deciding and afterwards in dealing with their too often unrecognised bereavement and possible guilt.

Little is known about the long term feelings of the parents or of the surviving children. Most parents say they made the right decision but also that there was insufficient respect for a loss of which, unlike a full termination, they have constant reminders in the surviving babies. Parents are often anxious about what, if anything, to tell the survivors and how they might react. Anger and blame towards the parents are possible but so are complex feelings of survivor guilt and of the arbitrariness of fate. Long term follow up studies of the parents but especially of the children are clearly needed.

*Guidelines for Professionals: Bereavement. London, MBF, 1997

Higher Multiple Pregnancies: Fetal Reduction. London, MBF, 1996

\section{S A TWIN STUDY OF CHRONIC FATIGUE}

Dedra Buchwald, Richard Herrell, Suzanne Ashton, Megan Belcourt, Karen Schmaling, Patrick Sullivan, Michael Neale, Jack Goldberg Correspondence address: Harborview Medical Center, 325 9th Avenue, Seattle, WA 98104, USA

Objective. The etiology of chronic fatigue syndrome (CFS) is unknown but genetic influences may be important in its expression.

Methods. A classical twin study using 146 female-female twin pairs, of whom at least one member reported $\geq 6$ months of fatigue, completed a questionnaire on CFS symptoms. Twins were classified using 3 increasingly stringent definitions: 1) chronic fatigue for $\geq 6$ months, 2) chronic fatigue according to the 1994 CDC CFS criteria not explained by medical conditions, and 3 ) chronic fatigue not explained by the medical or psychiatric exclusionary criteria of the CFS case definition. Pairwise and proband concordance rates in monozygotic and dizygotic twins were calculated for each definition of fatigue.

Results. The concordance rate for each definition of chronic fatigue is higher in monozygotic than dizygotic twins. For chronic fatigue not explained by the medical or psychiatric exclusionary criteria of the CFS case definition the concordance rates were $55 \%$ in monozygotic twins and $19 \%$ in dizygotic twins $(p=0.042)$. The estimated heritability in liability was $19 \%$ (95\% CI $0-56)$ for chronic fatigue $\geq 6$ months, $30 \%$ (95\% CI 0-81) for chronic fatigue not explained by medical conditions, and $51 \%$ (95\% CI 7-96) for a CFS-like illness not explained by medical or psychiatric conditions.

Conclusion. These results provide evidence for the familial aggregation of fatigue and suggest that genes may play a role in the etiology of CFS.

\section{P THE EARLY POSTNATAL GROWTH IN PRETERM TWIN BABIES}

\section{John Buckler}

Correspondence address: University Department of Paediatrics, Leeds General Infirmary, West Yorkshire LS2 9NS, UK

Twins are of low birth weight not only because they are more often born preterm, but also for any duration of gestation they are born with lower birth weights than singletons.

In this study twins born preterm were remeasured for weight and length when they had reached ages equivalent to 37 weeks and 40 weeks gestation. Their actual gestational ages ranged from 28 weeks for weights and 32 weeks for lengths up to 38 weeks. Extrauterine growth was as good as it would have been if continued within the uterus for length and weight at the equivalent of 37 weeks gestation and for length at 40 weeks irrespective of how preterm the babies had been born. At the equivalent of 40 weeks, the twins were heavier by about $200 \mathrm{~g}$ than if they 
had remained in utero until that time. This shows that from the point of view of early growth in weight and length twins are at no disadvantage from being born preterm, and the commonly held assumption that postnatal growth of preterm babies approximately parallels what would have occurred if they had remained in utero is correct for twins.

\section{S GROWTH AND DEVELOPMENT OF TWINS}

\section{John Buckler}

Correspondence address: University Department of Paediatrics, Leeds General Infirmary, West Yorkshire LS2 9NS

The weight, length/height and occipito-frontal circumference (OFC) of twins were measured serially from birth to 4 years. By one year boy and girl twins catch up singletons in weight remaining similar up to 2.5 years but subsequently twins tend to be lighter than singletons. The length/height of boy twins are similar to singletons from an early stage but female twins are slightly taller. OFC values of twins are smaller than singletons, particularly for girls. Measurements have also been undertaken on older twin pairs but mostly on one occasion only, through childhood. Data are presented as standard deviation scores (SDS) (sexes combined).

\begin{tabular}{llll} 
Group (No.) & Weight & Height & OFC \\
\hline Total > 2 yr (1897) & -0.19 & -0.09 & -0.43 \\
BW > 10\% (976) & +0.09 & +0.04 & -0.23 \\
BW 5-10\% (140) & -0.50 & -0.33 & -0.77 \\
BW < 5\% (182) & -0.74 & -0.47 & -1.04 \\
Age 2-5 yr (1313) & -0.09 & -0.07 & -0.41 \\
Age 5-10yr (424) & -0. & 0.10 & -0.55 \\
Age > 10 yr (312) & -0.57 & -0.25 & -0.42 \\
Twins with siblings (816) & -0.27 & -0.17 & \\
Siblings & +0.14 & +0.14 & \\
Mid-parental & & +0.10 & \\
\hline
\end{tabular}

Conclusions. The overall deficit in measurements of twins, which is greatest for OFC and least for height, is largely accounted for by those twins born light for dates. Deficits are least in the youngest age group $\&$ appear to increase through childhood.

\section{S HUMAN CLONES: WHICH SIDE ARE YOU ON?}

John Burn and Judith Goodship,

Correspondence address: Institute of Human Genetics, Newcastle UK

Monozygotic twins are natural clones and have long been recognised to have special problems associated with being "a malformation to whom Nature was kind". Monozygotic twins are susceptible to heart malformation. As an asymmetric organ, the heart is extremely sensitive to the determination of laterality. The increased incidence of isomerism sequence in the right half of conjoined human thoracopagus twins and the physical clues of disturbed laterality among twins discordant for heart defects led us to speculate that disturbance of the left right gradient associated with MZ twinning might be a mechanism leading to malformation, Levin et al supported this by showing in the chick that the product of the gene nodal could cross from the right side of one twin and convert the left side of its conjoined neighbour into a right side, thus making the right half of the pair isomeric. The last few years has seen an explosion of knowledge about the pathways leading to the determination of laterality. The inv gene isolated by the Newcastle group in collaboration with colleagues in USA and Japan is an early player; when defective the body plan is reversed. Loss of LR dynein can make laterality random while several other genes such as cryptic, lefty 1 and lefty 2 play critical roles in breaking symmetry and maintaining the left right gradient. Much interest is focused on the cilia of Henson's node and integrity of the midline structures initiated by primitive streak formation. As the molecular mechanisms unravel the impact of the MZ twinning process on this critical early process will be better understood and the original hypothesis, that twinning can cause isolated heart defects by upsetting the left right gradient, will at last be open to experimental examination.

\section{DISEASE CONCORDANCE AND GENETIC SUSCEPTIBILITY TO TYPE 1 DIABETES} IN ITALIAN TWINS

Raffaella Buzzetti, Paolo Pozzilli, Frida Leonetti, Umberto Di Mario, Rodolfo Cotichini, M. Antonia Stazi.

Correspondence address: Dept. Scienze Cliniche, Univ. of Rome "La Sapienza", Italy.

The identification of twins affected by Type 1 diabetes in a particular geographical area has important implications for disease pathogenesis. The incidence of Type 1 diabetes varies between $8 \times 100,000<15$ years of age in continental Italy and 35 x 100,000 < 15 years of age in Sardinia. In addition, one third of patients are diagnosed above the age of 15 years and approximately $10 \%$ are included in the group so called "Latent Autoimmune Diabetic in Adults" (LADA). Overall Type 1 diabetes should include approximately $10-15 \%$ of all cases of diabetes in Italy. In the Lazio region (central part of Italy including its capital Rome), the incidence of Type 1 diabetes is low compared to Sardinia and similar to the rest of continental Italy. According to the Italian Twin Registry, there are about 120,000 potential twins living in the Lazio region $(5,800,000$ inhabitants): considering a prevalence of Type 1 diabetes of $0.2 \%$, we can assume approximately 240 diabetic twins living in this region. Linking the Italian Twin Registry with the outpatient diabetic clinic database of the Lazio region (diabetes care - including syringes, glucometers, etc. - is free of charge in Italy, provided the patient is registered with the National Health Service) we will be able to identify all twins among the diabetes patients. The aim of our project is to calculate the concordance rates for Type 1 diabetes in MZ and DZ twins and to characterise twins according to age of disease onset and clinical features.

037F

\section{A POPULATION BASED STUDY OF MULTIPLE SCLEROSIS IN TWINS FROM CONTINENTAL ITALY AND SARDINIA}

Stefania Cannoni and Rodolfo Cotichini for the Italian Twin Study Group Correspondence address: Department of Neurological Sciences, University of Rome

"La Sapienza”, ITALY

Concordance rate studies in monozygotic vs. dizygotic twins provide a direct measure of the relative importance of environmental and genetic factors in the pathogenesis of multifactorial diseases. We took advantage of two unique opportunities that exist in Italy for such a study in Multiple Sclerosis (MS): the existence of the world's largest twin registry and the presence of populations with different genetic background and different disease prevalence. We performed a concordance rate study of MS in Italian twins by matching centralized medical databases (MS Centers, hospital discharges) with the Italian twin registry in order to select individuals with MS. So far, 25.000 patients with a diagnosis of MS (23.000 from continental Italy and 2.000 from Sardinia) have been screened and 170 twin pairs (157 from continental Italy and 13 from Sardinia) have been identified. An ad interim analysis of the results shows in continental Italy a probandwise concordance rate of $9 \%(2 / 41)$ in $\mathrm{MZ}$ and $3 \%(2 / 116)$ in DZ, while in Sardinia the rates were $40 \%$ (1/4) for MZ and $0 \%(0 / 9)$ for DZ. Compared with the majority of the published studies, the concordance in identical twins is low. If confirmed, this result may suggest that the relative contribution of environmental and genetic factors to the pathogenesis of MS may be diverse in different populations.

\section{S COMBINED LINKAGE AND ASSOCIATION ANALYSIS IN GENE DISCOVERY}

Lon Cardon

Correspondence address: Wellcome Trust Centre for Human Genetics, Oxford, UK

Success in identification of genetic loci for complex multifactorial traits has been limited, despite considerable efforts in positional cloning and candidate gene studies. A number of possible reasons have been described to explain these limited findings. In the linkage domain, the most likely explanation may simply involve insufficient power to detect genes of small effect and experimental error in genotyping and pedigree relationships. Association studies may also suffer from these limitations, but a number of additional factors are also clearly important. At particular interest at the moment are patterns of linkage disequilibrium across the genome. In the absence of understanding how LD operates with respect to 'anonymous' markers, it is extremely difficult to distinguish real association results from those differing by chance. Here I examine some of these patterns and their possible influence on association studies.

\section{S THE PAEDIATRICIAN'S VIEW ON THE EPIDEMIC}

\section{Manuel RG Carrapato}

Correspondence address: Hospital S Sebastião, Feira, Portugal

Assisted reproductive technologies (ART) although also increasing in Portugal, are fairly well-controlled, contrary to some other western 
countries, somewhat limiting the effects of the "epidemic". Firstly, medically-induced ovulation is only prescribed by obstetricians in recognised centres, thus curtailing the "over-the-counter" dispensing witnessed in some countries; the same applies to all other ARTs. At present, the number of embryos is, by consensus, limited to $2 / 3$, hence the days of very high-order pregnancies are over or are becoming very rare. Nevertheless, paediatricians are still faced with an increasing number of multiples and the related medical and social problems. Peri-neonatal mortality and morbidity due to prematurity, IUGR, the complications in the surviving neonate following the intra-uterine death of his/her partner(s), the feto-fetal transfusion syndrome, the neurosensorial sequelae, etc. are just some of the well-known medical burdens. On the socia side, when faced with higher-order deliveries, it is not unusual, to be short of places with one or more of the neonates being subjected to transferral elsewhere, giving rise to obvious parental inconvenience and distress. A similar situation occurs when surgical complications, for instance, necessitate splitting multiples between different hospitals sometimes many miles apart. Bereavement and family counselling are additional and complicated matters not always handled with skill and sensitivity. Furthermore, how many paediatric clinics are there specialised in the follow-up of multiples from infancy to adolescence and what can these children and their families expect from us, the Paediatricians? Finally, what are the financial implications and can we, as a society, afford them?

\section{S PERI-NEONATAL STEROIDS IN MULTIPLES}

\section{MRG Carrapato}

Correspondence address: Hospital São Sebastião, Portugal

Since the early works of Liggins \& Cols. 30 years ago showing that Betamethasone greatly reduced neonatal deaths and RDS in very preterm infants with no deaths from HMD and IVH, the use of antenatal steroids was gradually established for the induction of fetal lung maturation. In addition, other beneficial effects have been claimed, namely in the prevention of NEC and PVL and as recently as 1994 the National Institute of Health Consensus recommended that "... ANCS be used for delivery anticipated before 34 weeks with intact membranes and before 32 weeks with ruptured membranes". However, in recent years questions have been raised regarding their efficiency in pregnancies complicated by hypertension, PROM, IUGR, and also regarding the number of cycles to be administered, which steroid, the side-effects upon the mother and, especially, the possible unfavourable outcome for the fetus and the child. Indeed, besides the immediate (often transient) undesirable effects of raised blood pressure, LVH and adrenal suppression, concern rests now upon weight and growth restriction and neurosensorial impairmen in the older child, at least following repeated courses. Trying to reach a balance between the positive action of antenatal steroids and potential complications in the long run, it is reasonable to recommend one course of ANCS whilst a second course should await the results of ongoing trials.

Postnatal steroids are also fraught with similar problems of immediate and undesirable side-effects from glucose intolerance to hypertension, adrenal suppression and the more-serious, although unusual, gastric haemorrhage and perfuration. The major concern is also long-term neurological handicap similar to that from antenatal steroids. However postnatal steroids have been shown to promote early extubation and to reduce CLD and therefore they might have a role to perform if neonatal survival appears unlikely without them. As to the timing for administration, it is reasonable to recommend their use between the first and second week of life in a ventilator-dependent neonate at the lowest possible dose and for the shortest possible time. If the present state of affairs is rather unclear for single pregnancies, the issue becomes even more complex in multiple pregnancies. Although twins mature earlier than singletons, the question is at what gestational age do they start to accelerate their maturation? Is there a difference in maturation for mono- and dizygotic twins? Will gender matter and what if they are of different sexes? Assuming that steroids will be useful, what then will be the recommended dose? The same as for singletons, or half, or double the dose? How will it be known that the dose has been equally distributed between each of the fetuses regardless of their individual fetoplacental units? And in the case of higher-order pregnancies, will not these questions, and many others, assume even greater relevance, and what are the answers or the alternatives?

\section{F A NEW MZ-ONLY DESIGN: X CHROMOSOME INACTIVATION AND THE FEMALE SURVIVAL ADVANTAGE}

Kaare Christensen, James W.Vaupel, Karen Helene Ørstavik

Correspondence address: Institute of Public Health, Epidemiology, Sdr. Boulevard 23A,

DK-5000 Odense C.KChristensen@ health.sdu.dk

Traditionally, studies of monozygotic twins alone cannot be used for addressing the influence of genetic factors. However, we have recently shown that studies of elderly female $\mathrm{MZ}$ twin pairs provide evidence that X-linked genetic factors are affecting cell proliferation/ survival. $\mathrm{X}$ inactivation makes females mosaics for two cell populations, usually with an approximate 50:50 distribution. A skewing of this distribution in peripheral blood cells is more common among elderly females. Depletion of hematopoietic stem-cells followed by random differentiation may explain the acquired skewing with age. However, an animal model suggests that selection processes are involved which are based on Xlinked genetic factors. We studied peripheral blood cells from 71 monozygotic female twin pairs aged 73-93, and 33 centenarians, and found that with age one of the cell populations becomes predominant for most females. We also observed a strong tendency for the same cell line to become predominant in two MZ co-twins. This suggests that $\mathrm{X}$-linked genetic factors influence human hematopoietic stem-cell kinetics. The fact that females have two cell lines with different potentials could be one of the reasons why women live longer than men.

\section{P}

\section{MULTIMETHOD ASSESSMENT OF THE TWIN RELATIONSHIP}

Francesca M. Cimino, Laura A. Baker,Jennifer K. Johnson, Adrian Raine Correspondence address: University of Southern California, Department of Psychology, 3620 S. McClintock Ave \#501, Los Angeles, CA 90089-106,

Twin studies provide a unique opportunity to understand the nature of sibling relationships. The present study utilized both parent and child reports of the negative and positive aspects of each twin's behavior towards the other. Observational data are also obtained, based on a 10minute videotape interaction of the twins performing a contrived task. These methods yielded multiple measures of warmth, conflict, rivalry, and dominance of each twin. Correlations among child reports, maternal reports, and observational measures are examined to evaluate the extent of agreement across raters and methods. Comparisons are also made between monozygotic and dizygotic twin pairs, as well as between samesex and opposite sex pairs, to evaluate the extent to which zygosity and gender influence the twin relationship.

\section{F DEVELOPMENT AND REPRESENTATIVENESS OF A POPULATION-BASED COHORT OF TWINS}

Myles Cockburn, Ann Hamilton, Wendy Cozen, Thomas Mack.

Correspondence address: USC/Norris Cancer Center, 1441 Eastlake Ave, MC9175, Los Angeles CA 90089-9175

We have established a large cohort of twins to facilitate studies of the role of genetics and environment in the development of disease. We report here on the establishment of this cohort, now in excess of 41,000 native California twins, whose enrollment continues. The cohort was obtained by linking the birth records of the state of California with the State's Department of Motor Vehicles database to determine a current address for all multiple births between 1908 and 1972 (200,589 individuals). To date, this linkage has revealed 112,468 matches, and was less successful in older females than in younger females and all males. We have sent a detailed 16-page question naire to 102,258 individuals and have received responses from over 41,000. Based on various estimates of numbers of individuals not receiving a questionnaire, we estimate our crude response rate to be between $42.2 \%$ and $49.6 \%$, highest among females in their 40s $(62.8 \%)$. Compared to the 1990 resident population of California-born resident singletons, the respondents were of similar age, sex, race and residential distribution (for although we were able to locate fewer older females, they had a higher response rate), but were substantially less likely to have more than 12 years of education.

\section{P EFFECTS OF PRENATAL EXPOSURE TO TESTOSTERONE ON COGNITIVE PERFORMANCE AND BEHAVIOUR: A TWIN STUDY}

Celina C.C. Cohen, S.H.M. van Goozen, P.T. Cohen-Kettenis \& J.K. Buitelaar

Correspondence address: Department of Child and Adolescent Psychiatry, University Medical Center Utrecht, P.O. Box 85500, 3508 GA Utrecht, The Netherlands. C.Cohen@psych.azu.nl

Research has shown women and men differ on various cognitive and behavioural aspects. The answer to which mechanism is responsible for these differences is a complicated one. Although for many years research on gender differences in humans has been dominated by social-role theories there is increasing support for the notion that prenatal exposure to gonadal hormones has clear effects not only on physical differences between males and females, but also on gender differences in cognition and behaviour. In animal research it has been shown that exposure to gonadal hormones is influenced by intrauterine position and that foetuses that are located between two male foetuses are exposed to higher levels of testosterone than foetuses that are situated between two female or one female and one male foetus. In our study we examine the presumed prenatal influence of various exposure levels of testosterone on gender differences. 84 girls from opposite-sex (OS) and 60 girls from same-sex (SS) twin pairs are compared on various cogni- 
tive, behavioural and physical aspects at the age of 10. It is expected that OS girls and SS girls differ in performance on these various gender sensitive tasks, and that OS girls in particular show a pattern of performance away from the typical female pattern as a result of prenatal exposure to higher levels of testosterone. The poster will present the results and discuss the implications of the findings.

\section{$045 \mathrm{~F}$ \\ MUTLIPLE BIRTH FAMILIES THEIR NEEDS AND EXPERIENCES}

\section{Hilary Collins}

Correspondence address: Tamba in Northern Ireland, 216 Belmont Road,

Belfast nioffice@tambani.org

The poster will summarise the key findings of a survey carried out by Hilary Collins, NI Co-ordinator in the Northern Health and Social Services Board (NHSS) area. Tamba received a non-recurrent donation of $£ 5000$ from the NHSS Board to provide early and regular support to children and their families in the board area, shaped by an assessment of their needs. The funding was used to carry out a survey to gather the primary information on the needs and experiences of families with multiple birth children aged 0-5 years. This survey was the first time Tamba had had the opportunity to contact families outside the membership with children up to five years in an attempt to elicit and document their views. The survey incorporated both qualitative and quantitative methods of data collection, with the information obtained from focus groups forming the basis of the questionnaire. Health Visitors forwarded the questionaire to 301 families identified on their caseloads; 25.9 per cent were returned for analysis using SPSS software. The findings showed a lack of information available to families about a multiple pregnancy. Other issues raised included a lack of understanding from health professionals abou the unique difficulties faced by families with multiples, and lack of statutory help available. Most families faced financial burdens and stress associated with having a multiple birth family.

\section{F A RETROSPECTIVE POPULATION BASED STUDY OF TWIN BIRTH WEIGHT DISCORDANCY}

\section{Christine E. Conner and Doris M. Campbell}

\section{Correspondence address: Dept of Obstetrics, Aberdeen Maternity Hospital,}

Aberdeen AB25 2ZL, UK

Twin birth weight discordancy is usually defined as birth weight difference expressed as a proportion of the larger twin's birth weight No accepted cut off exists and it would be useful to identify the discordancy threshold at which a significant difference in perinatal outcome occurs. Aberdeen Maternity Hospital (AMH) is in a unique position being the only obstetric centre for a large area where all twins deliver. Additionally, Aberdeen Maternity and Neonatal Databank, contains information on obstetric events in women attending AMH from 1949 The aims were to identify factors predisposing towards twin growth discordancy and the effects of different degrees of discordancy on perinatal outcome. Twins delivering $\geq 24$ weeks, from 1949-1998, were selected, percentage birth weight discordancy calculated and analysed in quintiles $(<10 \%$ to $\geq 40 \%)$ using SPSS. Of the 2552 twin sets identified, 71 wer excluded. No predisposing maternal or obstetric factors were identified and no differences in fetal sex, zygosity or chorionicity were eviden within the different discordancy categories. Percentages with low Apgars became significantly higher for both twins from a birth weight discordancy of $\geq 20 \%$ (twin $1 p<0.05$; twin $2 p<0.01$ ). Perinatal mortality (PNM) rate for twin 1 increased significantly from a birth weight discordancy of $\geq 30 \%(p<0.05)$ whilst for twin 2 this was significantly increased from a discordancy of $\geq 20 \%$ ( $p<0.001)$. Using multivariate analysis, birth weight discordancy was the major factor influencing PNM in either twin becoming significant at a discordancy of $\geq 20 \%$ $(p<0.001)$. A suitable definition for significant birth weight discordancy is an intra-twin birth weight difference of $\geq 20 \%$.

\section{F STATUS EPILEPTICUS IN A VIRGINIA TWIN SAMPLE}

Linda A. Corey and John M. Pellock, Virginia Commonwealth

Correspondence address: University, Richmond, Virginia U.S.A.

Twin studies provide an extremely efficient means of determining if genetic factors contribute to disease susceptibility, particularly in the case of relatively rare disorders such as status epilepticus (SE). Information on SE in 10,915 Virginia-born twin pairs identified from the population-based Mid-Atlantic Twin Registry was obtained in order to characterize SE in this group and determine if genetic factors contribute to its risk of occurrence. In this study, SE was defined as any single seizure or intermittent seizures from which the individual did not regain consciousness lasting 30 minutes or longer. History of seizures and SE were validated using medical records and by detailed personal or parental interviews. The prevalence of SE in this population was found to be 327 per 100,000 twins. At least one episode of SE was validated in $70(18.4 \%)$ of the twins in whom a history a seizures could be verified. Among the 22 monozygotic (MZ) and 44 dizygotic (DZ) twin pairs with verified SE, age at first SE occurrence ranged from two months to 59 years. All SE-concordant pairs were MZ. Age of onset of SE in concordant pairs differed by, as much as, 16 years and one pair was discordant for both SE seizure and epilepsy types. Probandwise concordance rates for SE in this sample were 0.31 for $\mathrm{MZ}$ and 0.00 for DZ twins, overall. When this rate was calculated for $\mathrm{MZ}$ twin pairs who were also concordant for a history of seizures, probandwise concordance for SE increased to 0.53 .

These results provide an estimate of SE in a defined population and afford further evidence that genetic factors make a contribution to SE risk that appears to be independent of risk for seizures.

\section{P PROGRESSION TO COELIAC DISEASE IN ITALIAN TWINS}

Rodolfo Cotichini, Luigi Greco, Romina Romino, lolanda Coto, Roberto Tosi, M. Antonia Stazi.

Correspondence address: Istituto Superiore di Sanità, Rome, ITALY

The main objective of this study was to estimate the progression to celiac disease of dizygotic and monozygotic twins according to their HLA status. Through record linkage with the Italian Twins Register and the membership list of the Southern branch of the Associazione Italiana Celiachia, 58 twin pairs were ascertained (21 MZ, $37 \mathrm{DZ})$. Forty-five pairs were further screened for EMA and tTGASE antibodies, their zigosity was confirmed by DNA fingerprinting and each twin was typed for molecular class II HLA DRB1 and DQB1, thus confirming $19 \mathrm{MZ}$ and 26 DZ pairs. In $10 \mathrm{MZ}$ pairs and one $\mathrm{DZ}$ pair $\mathrm{CD}$ was diagnosed in both members within one year. Overall MZ twin siblings had a higher risk of progression to $C D(14 / 19,6$ diagnosed at screening) as compared to $\mathrm{MZ}$ twin siblings $(3 / 29,1$ diagnosed at screening). By life table analysis the probability of developing $\mathrm{CD}$ was higher among the monozygotic twin siblings $(43.7 \% \mathrm{v} 8.9 \%)$ at 10 years of discordance; $p<0.05$. MZ and DZ pairs were also analysed according to HLA genotype at risk. Twelve of 16 DQ8/DQ2 MZ twin siblings developed CD, as compared to $2 / 3$ not DQ8/DQ2 MZ twin siblings. As regards DZ twin siblings, 3/17 DQ8/DQ2 non-index twin siblings developed CD versus 0/9 not DQ8/DQ2. Considering only the DQ8/DQ2 twin siblings, the probability of developing CD within 10 years was $37.0 \%$ in $\mathrm{MZ}$ v. $13.8 \%$ in DZ; $p<0.05$. Adjusting by DQ8/DQ2, monozygotic and dizygotic twins differ in progression to $\mathrm{CD}$, suggesting the important but not unique contribution of the HLA at risk region to disease onset.

\section{P EFFECT OF TOBACCO SMOKE ON CYTOKINES IN TWINS}

Wendy Cozen, Myles Cockburn, James Gauderman, Kathleen Danley, Nicole Stroud, Thomas M. Mack.

Correspondence address: USC Norris Cancer Center, 1441 Eastlake Ave., MS \# 44, Los Angeles, CA, USA 90033

Tobacco smoke is responsible for causing numerous asthmatic episodes each year in both children and adults. It is not known how tobacco smoke produces this effect, but it may be mediated through the immune system. The results of one study suggest that smokers have higher levels of interleukin-4 (IL-4), inter-leukin-5 (IL-5) and interleukin-13 (IL-13 IL-4, IL-5 and IgE compared to non-smokers, implying that tobacco smoke works through the immune system to provoke asthma. However, the study did not adjust for gender, race, and age. We have proposed to repeat the study as a natural experiment in identical twins discordant for smoking (one smokes and the other does not), comparing their IL-4, IL-5 and IL-13 levels. Because the twins are matched on age, race, gender, genetics and early environ-mental exposures, if we do find a difference in their cytokine levels, we can more confidently attribute the difference to adult smoking behavior. Twins are recruited from the California Twin Program. Smoking history is obtained from questionnaires previously filled out by the twins and updated during the telephone interview. Blood specimens are obtained from twins by their physicians and mailed to us via overnight courier. Peripheral blood mononuclear cells are isolated from the whole blood, cultured and stimulated with PHA. The supernatant is collected and commercial ELISA assays are performed. To date, we have collected specimens from 168 individuals (84 pairs) and have ELISA results for IL-4 from 65 pairs. Preliminary results suggest that smokers have significantly higher IL-4 levels than their non-smoking identical twin $(p<0.025)$. Results for all cytokines will be presented. 
050P

\section{SUSCEPTIBLE HD CYTOKINE PHENOTYPES IN TWINS}

Wendy Cozen, Rizwan Masood, Parkash Gill, Myles Cockburn,James

Gauderman, Kathleen Danley, Nicole Stroud, Thomas M. Mack.

Correspondence address: USC Norris Cancer Center, 1441 Eastlake Ave., MS \# 44, Los Angeles, CA, USA 90033

Environment plays a role in HD risk but genetic factors also contribute. We hypothesized that Th2/Th1 skewing was responsible for the increased risk of HD in identical twins since

HD cases have normal humoral but depressed cellular immunity. Healthy identical twins of HD cases serve as genetically identical predisease surrogates in which to measure possible susceptibility phenotypes unaffected by disease. We examined Th1 (interleukin-12 \{IL-12\}, interleukin- 2 IL-2\}, interferon-g $\{$ INF-g $\}$, and tumor grow th factor- $\beta$ $\{$ TGF- $\beta\}$ ) and Th2 (interleukin-6 \{IL-6\}, interleukin-8 $\{$ IL-8\}, and interleukin-10 \{IL-10\}) levels in 54 unaffected identical twins of HD cases compared to those of age and race matched controls using an analysis of variance for unequal sample size and adjusted for gender and age strat ifying by zygosity and histology. While no significant differences between cotwins' and controls' cytokine levels were observed, cotwins of mixed cellularity (MC) cases and lymphocyte predominant cases (LP) HD cases had significantly lower levels of IL-12 (both unstimulated and PHA-stimulated) compared to matched controls. Cotwins of MC cases also had significantly lower levels of INF-g but higher levels of IL-6. Cotwins of nodular sclerosis (NS) HD cases had significantly higher levels of unstimulated INF-g compared to matched controls. PHA-stimulated cytokine levels were uni-formly, but not significantly, higher in cotwins of NS cases compared to controls. Thus, an imbalance in the Th2/Th1 ratio appears to be present in healthy twins of MC and LP HD cases and may increase susceptibility to these types of HD.

\section{P ASCERTAINMENT OF TWINS IN THE WESTERN AUSTRALIAN TWIN REGISTER USING RECORD LINKAGE}

Maxine L Croft, Nicholas H de Klerk, Janice Hansen, Phyllis T Alessandri. Correspondence address: TVW Telethon Institute for Child Health Research, PO Box 855, West Perth, 6872, Western Australia, Australia.

Objectives. To describe a) the methods of construction of the populationbased West Australian (WA) Twin Register and b) the maternal twins identified through this process.

Methods. The WA Twin Child Health (WATCH) study uses data from the WA Twin Register of multiple births born in WA from 1980-1992 inclusive. Families with a multiple birth were ascertained through probabilistic record linkage of birth records to form maternally linked sibships.

Results. The linkage process led to the identification of women who appear to be twins and/or siblings. Population rates of multiple births in the 1960s would result in an expected 543 sets of same sex twins. Linkage found $305(56 \%)$ sets of apparent maternal twins. Of those linked maternal twins, $22(31 \%)$ out of the 72 nulliparous twin mothers, had their first birth within one year of a birth to their co-twin and 54 $(75 \%)$ had at least one birth in the same hospital. Of the 2,848 WATCH study responder mothers, $60(2 \%)$ are twins, with $26(43 \%)$ monozygotic $20(33 \%)$ dizygotic (DZ) same sex and $14(23 \%)$ DZ male-female twins.

Conclusions. Probabilistic record linkage is a viable method of ascertainment of twins for a Twin Register and can result in maternal twins and siblings being identified.

\section{P TWINS IN MAYA MYTHOLOGY}

David Czukerberg, Louis G. Keith*, Joseph Blankstein, Donald M. Keith*. Correspondence address: Mount Sinai Hospital $\backslash$ Finch University, Dept. OB\GYN California Ave. at 15th St, Chicago, IL 60608. * The Center for Study of Multiple Birth.

Objective. To describe the theme of twinship in the Popol Vuh.

Results. The Popol Vuh, the most important text in the native languages of the Americas, narrates the cosmogony, mythology, traditions and history of the Quiche Indians, a Maya tribe that lived in the highlands of Guatemala in pre-conquest era. Three sets of twins are mentioned. Hun-Hunahpu and Vucub-Hunahpu, first set, are summoned to play ball with the Lords of the Underworld, Xibalba, and are killed by the death gods. The head of Hun-Hunahpu is hung in a calabash tree. One day Xquic, a daughter of one the death gods, walked past the calabash tree and the desiccated head spat into her hand. She magically became pregnant, fled into the Middleworld and gave birth to the Hero Twins, Hunahpu and Xbalanque, second set. The Hero Twins grow up, defeat various opponents including their jealous rival older pair of twin half brothers: Hun-Chuen and Hun-Batz, third set, by transforming them into monkeys. The Hero Twins are summoned to the Underworld to play ball. They overcome the Lords of Xibalba by a series of tricks, ending in the destruction of the death gods. The Hero Twins then dug up the bodies of Hun-Hunahpu and Vucub-Hunahpu and revived them, after which they rose up to the sky to become the Sun and the Moon.

Conclusions. Twins are a central and recurring theme in Maya mythology and represent one of the earliest religious beliefs of mankind.

\section{P ZYGOSITY DETERMINATION} WHY IS IT SO IMPORTANT?

\section{Margie Davies}

Correspondence address: Multiple Births Foundation, Queen Charlotte's

and Chelsea Hospital, Du Cane Rd, London W12 OHS

Throughout history the origin of twins has provoked intense interest and speculation and it is only relatively recently that scientific research has clarified their development. It is medically important to establish chorionicity - so monochorionic twins can be closely monitored for conditions such as twin to twin transfusion syndrome (TTTS). Chorionicity should be determined routinely in the first trimester of pregnancy, besides the medical reasons there still appears to be an underestimation of the need for parents and children themselves to know their zygosity. Parents are still being told in some cases that if their twins are dichorionic, then the babies cannot be identical. In fact one third of monozygotic twins are dichorionic. Until the development of DNA testing the examination of certain markers in the blood could give a result of up to $99 \%$ accuracy of monozygosity, but now the new DNA testing procedures are more reliable and fortunately, though still expensive, increasingly widely available. All the parents who contacted the Multiple Births Foundation (MBF) for zygosity testing were asked what information they had received regarding chorionicity and zygosity, and particularly whether they were told that their dichorionic twins were not identical. The MBF promotes the need for professional education regarding zygosity determination and is seeking to establish the availability of routine testing in all maternity units.

\section{P FEASIBILITY OF USING BIRTH RECORDS FOR RECRUITING OLDER TWINS FOR THE SRI LANKAN TWIN REGISTRY}

DA Rohana K Dayaratne,Athula Sumathipala,Devaka JS Fernando, Sisira H Siribaddana, Nimali de Silva, Nihal Abeysinghe.

Correspondence address: National Twin Registry, Department of Medicine,

Sri Jayewardenepura University, Nugegoda, Sri Lanka.

To expand the National Twin Registry of Sri Lanka to a population based register, we examined the feasibility of tracing older twins by inspecting birth records and recruiting them by postal invitation and in-person contact.

Methods. Birth records at a divisional secretariat reported from 2 maternity hospitals between the years of 1954-1970 were scrutinised randomly to identify twins. These 2 hospitals had the highest twin delivery rates for the whole country. We identified 310 twin pairs and a postal questionnaire was sent. Research assistants visited a cohort of non-respondents (71) in the postal survey.

Results. 620 twins were identified after perusing 20,700 birth records. Estimated twining rate, was 14.98 twin births for 1000 registered births for a year. In the postal survey, $37(12 \%)$ responded and 62 letters were returned $(20 \%)$, as twins were no longer in the postal address. Both were living in 20 pairs, one each in 15 pairs, and both dead in 2 pairs. In the field visits, $42(59.2 \%)$ addresses were located. Information was available on 16-twin pairs Both were living in 8 pairs, one each in 4 pairs, and both dead in 4 pairs. At least one twin was traced in 10 pairs (14\%). Both postal and field survey gave a low yield. This finding is different from tracing younger twins born between 1985-1997 by using the same methods. Migration, urbanization and development in the country may have affected tracing older twins' from the birth record addresses, which are decades old.

\section{F BIRTH WEIGHT AND TRACKING OF BLOOD PRESSURE: A 15-YEAR FOLLOW-UP STUDY OF DUTCH ADOLESCENT TWINS}

Eco de Geus, Richard ljzerman, Caroline van Baal, Elles Mulder, Jessica Hebly, Danielle Posthuma, Dorret Boomsma

Correspondence address: Dept of Biological Psychology, Vrije Universiteit Van der Boechorstraat 1, 1081 BT Amsterdam, The Netherlands

In a sample of 17-year-old Dutch twins, we observed an inverse relation between birth weight and blood pressure. Blood pressure was measured in the laboratory during rest and mental stress conditions; birth weight was obtained from the mothers of twins. Intrapair differences in birth weight were negatively associated with differences in systolic BP in DZ, but not in MZ twins (Yzerman et al., 2000), suggesting genetic mediation of the association between birth weight and blood pressure. Fifteen years later, the twin sample was approached to participate in a laboratory study of brain 
and memory function, during which blood pressure was also assessed. Of the original sample of 320 twins 185 subjects participated again.

First results show substantial tracking for systolic blood pressure (correlations over time between 0.3-0.4) and for diastolic blood pressure (correlations over time between 0.4 and 0.5 ). We will look at the tracking data for blood pressure as a function of birth weight and address the question if the stability in blood pressure is caused by long-lasting effects of birth weight.

\section{F RATES OF HOSPITAL ADMISSION FOR CHILDHOOD ASTHMA AMONG AUSTRALIAN TWINS AND SINGLETONS.}

Nicholas de Klerk, Janice Hansen, Maxine Croft.

Correspondence address: TVW Telethon Institute for Child Health Research,

100 Roberts Road, Subiaco, WA 6008, Australia.

Background. A recent observational study in Scotland indicated a reduced rate of admission to hospital for asthma in children who were twins. Other studies have supported this finding, interpreted as demonstrating the protective effect of large families on allergic disease.

Aims. To estimate the relative hospital admission rate for twin births compared with singleton births.

Methods. Data came from the Maternal and Child Health Research Database of Western Australia (WA) which includes physical and sociodemographic characteristics of all mothers giving birth in WA from 1980 to 1995 (and beyond), with information about pregnancy complications, perinatal details, all causes of deaths (from 20 weeks gestation onwards), and details on hospital in-patient morbidity for all children who attend WA hospitals. Cox regression was used to estimate relative first admission rates for asthma as coded on the hospital record, in terms of mothers' age, parity, and multiplicity and the calendar year of birth

Results. Contrary to the Scottish findings, there was a $14 \%(95 \% \mathrm{CI}$ $5-22 \%$ ) increase in rate of admissions among twins, which increased to $25 \%(95 \%$ CI $14-36 \%)$ after adjustment for year, race (Indigenous or not), sex, birth order, and maternal age. Analysis including multiple admissions gave similar results. Rates of admission also increased steadily with increasing family size.

Conclusions. These findings are totally dissimilar to those found in Scotland, where however, admission rates to hospital for asthma in this age group are over 3 times lower than in WA.

\section{F EVIDENCE FOR SHARED GENES BETWEEN INSULIN RESISTANCE AND HAEMOSTASIS}

Marlies de Lange', Robert AS Ariëns ${ }^{2}$, Peter J Grant ${ }^{2}$, Tim D Spector' and Harold Snieder

Correspondence address. 'Twin Research \& Genetic Epidemiology Unit, St Thomas'

Hospital, London, UK. ${ }^{2}$ Academic Unit of Molecular Vascular Medicine, General Infirmary Leeds, UK

A number of components of the haemostatic system are associated both with risk of coronary heart disease and the insulin resistance syndrome. The extent to which genetic and environment influences contribute to this association is unknown. The aim of this study was to identify the contribution of common genes or common environment to the association of insulin resistance (IR) with levels of fibrinogen, plasminogen activator inhibitor (PAI) and tissue plasminogen activator (tPA). A total of 110 monozygotic and 301 dizygotic Caucasian female twin pairs, aged 18-74 participated in this study. IR was calculated according to the HOMA model (fasting insulin $\mathrm{x}$ glucose/22.5). Multivariate genetic model fitting techniques were carried out using the structural equation package Mx. The phenotypic correlation of IR with fibrinogen, PAI and tPA were 0.29 , 0.36 and 0.29 , respectively. A Cholesky decomposition model explaining the (joint) variance of IR, fibrinogen, PAI and tPA with additive genetic and unique environmental components fitted the data best. The genetic correlation of IR with fibrinogen, PAI and tPA was $0.39,0.42$ and 0.32 respectively. Environmental correlations were 0.19, 0.27 and 0.26.

The association between IR and haemostatic factors is due to effects of shared genes and shared environment, which will help to identify key pathologic processes involved in IR

\section{P \\ THE GENETICS OF TUNE DEAFNESS} DON'T BLAME THE PIANO TEACHER

Marlies de Lange', Dennis Drayna²,Ani ManichaikuR', Harold Snieder', Tim Spector

Correspondence address: 'Twin Research \& Genetic Epidemiology Unit, St. Thomas Hospital, London, UK. ${ }^{2}$ National Institute on Deafness and Other Communication Disorders, National Institutes of Health, Rockville, Maryland, USA

Background. We all know someone who cannot carry a tune, but acts like a Prima Donna. Previous studies suggested that about 1 in 20 people are tune deaf and there is considerable variation. This twin study investi- gates the genetic and environmental contributions to differences in musical pitch perception abilities.

Methods. A Distorted Tunes Test (DTT) was administered to a total of $136 \mathrm{MZ}$ and $148 \mathrm{DZ}$ Caucasian female twin pairs aged 18 to 74 The twins were asked to judge whether 26 simple popular melodies contained notes with incorrect pitch. Contingency tables were produced for the MZ and DZ twins using the number of correctly classified tunes (scores ranged widely from 9 to 26). Genetic model fitting techniques using the structural equation modelling package $\mathrm{Mx}$ were applied to obtain estimates of the genetic and environmental factors.

Results. Tetrachoric correlations were estimated at 0.67 for $\mathrm{MZ}$ and 0.44 for DZ twins. Subsequent model fitting showed that the best fit to the data included an additive genetic and a unique environmental component. The heritability was estimated at $71 \%$ (95\% CI: $61 \%-78 \%)$. There was no effect of shared environment.

Conclusion. Variation in musical pitch recognition is primarily due to highly heritable differences in auditory function.

\section{S}

\section{MONOZYGOTIC TWINNING RATE AFTER OVULATION INDUCTION}

Catherine A. Derom, Robert M. Derom, Robert F.Vlietinck

Correspondence address: Center for Human Genetics, Kapucijnenvoer 33 bJ, B-3000 Leuven, Belgium

While more and more authors have no doubt that the monozygotic twinning frequency is increased in association with artificial reproduction technology (ART), many questions about this unexpected phenomenon remain unanswered. The magnitude of the increase varies substantially according to the nature of the samples studied and the type of ART (ovarian stimulation alone, IVF and related procedures, timing of the transfer etc.). Most of the studies in the literature rely on hospital-based data and on chorionicity rather than on zygosity. This latter may underestimate the incidence of zygotic splitting as one third of pairs are dichorionic in spontaneous monozygotic twin pregnancies.

This study reports on the monozygotic twinning rate after different assisted reproductive treatment modalities and different drugs to induce the ovulation in a population-based registry of multiples with known zygosity and chorionicity. Since 1964 the East Flanders Prospective Twin Survey collects data on all the multiples born in the province of EastFlanders, Belgium. Between 1976 and 20001346 twin pairs and 127 triplet sets were born after ART. Basic perinatal data, placentation and zygosity were recorded at birth. Of the 1346 twin pairs $83 \%$ are dizygotic, $4 \%$ monochorionic and $13 \%$ dichorionic with still unknown zygosity. Of the 127 triplet sets, $83 \%$ are trizygotic, $11 \%$ dizygotic, $1 \%$ monozygotic and 5\% trichorionic with unknown zygosity. Additional tests are being performed to assess the zygosity of those pairs with no reliable zygosity information.

\section{P \\ STANDARDIZED POPULATION-BASED} VITAL STATISTICS OF TWIN BIRTHS

Robert Derom, John Kiely, Louis Keith, Elizabeth Bryan

Correspondence address: International Society for Twin Studies (ISTS),

c/o Queensland Institute of Medical Research, 300 Herston Road, Brisbane 4029, Australia

Both multiple births associations and scientists interested in multiple pregnancies are eager to compare basic perinatal twin data from different countries or regions. To enhance the collection of comparable data the following simple protocol is proposed:

I. Country or region

II. Short description of population

1. Number of inhabitants

2. Setting

3. Total number of births Singletons

Twins

Higher order multiple births

III. Perinatal basic data on twin maternities

1. Origin of (provider of) the data

2. Prevalence of twin maternities

3. Numbers of same- and opposite-sex pairs

4. MZ/DZ proportions according to Weinberg's rule

5. Fetal and early neonatal deaths (separately for SS or OS pairs?)

IV. Correspondence (address; fax and telephone; email; website) The data should be collected in a uniform way, that is by using terms and definitions recommended by WHO and FIGO (Fédération Internationale de Gynécologie et Obstétrique). 
Recent statistics from different sources will be presented. The idea is to extend the number of participating centres and institutions in the future and to publish yearly the results in Twin Research, the official journal of ISTS.

\section{P}

\section{EARLY REPORT ON THE AVAILABILITY OF ISLAND WIDE TWIN DATA IN THE CENTRAL BIRTH REGISTRATION DEPARTMENT}

Nimali de Silva,Athula Sumathipala,Devaka JS Fernando, Nihal Abeysingha, Sisira H Siribaddana, DARK Dayaratne, Deepthi De Silva, Narada Warnasuriya Correspondence address: National Twin Registry, Department of Medicine,

Sri Jayewardenepura University, Nugegoda, Sri Lanka.

Population based twin registers are not common outside Scandinavia, particularly in the developing world. Sri Lanka has a $98 \%$ accuracy of birth registration. However only since 1992 these records have been computerized at the Central Birth Registration Department including the details of the twin births. These details are complete only up to 1997.

We scrutinised those computerized records for the year 1992, to look into the feasibility of using it to obtain a twin database. A total of 3189 twins were identified out of 356,842 births registered at the at the Registrar General Department for the year 1992, giving a twining rate of 8.93 per 1000 registered births. Higher numbers of twins were registered from major urban centers where the Teaching, Maternal, General, Provincial and Base hospitals are situated. This finding was consistent for the whole country. It was evident that there is a large population based twin database for the whole country from 1992-1997. 1998-2000 data is not yet computerised. To obtain a cohort of twins since birth was a possibility provided that there was a facility to get all the prospective birth from now onwards. Support of the statutory services was thus requested and was granted. Therefore all prospective twin births will be directly reported to the Twin Registry, at the time of birth registration. This prospective birth cohort for the whole country will be available for many different projects in the future.

\section{S SEX REASSIGNMENT IN MZ MALE TWINS (THE JOHN/JOAN CASE) AND WHAT THIS MIGHT TELL US ABOUT SEXUAL IDENTITY}

\section{Milton Diamond \\ Correspondence address: University of Hawaii, John A. Burns School of Medicine,} 1951 East-West Road, Honolulu, HI 96822, USA.

The so-called John/Joan case has provoked new challenge s to the saliency of rearing as being deterministic in one's sexual identification as male or female. Aspects of how these twins interacted and compared and contrasted themselves with each other and with peers provides insight as to some factors in the development of sexual identity. Comparison is made as to how others, for example, transsexual, and intersexed persons, twin or not, and typical singletons, come to understand their identity. Consideration is given to various factors that have been purported to thwart the salient and supposedly "overwhelming and deterministic" forces of rearing are also discussed. Such factors include: maternal and paternal influences, siblings and peers, secrecy as to original sex, indoctrination and brow beating.

\section{S TWINS: WHAT THEY MIGHT TEACH US ABOUT THE DEVELOPMENT OF SEXUAL IDENTITY}

\section{Milton Diamond \\ Correspondence address: University of Hawaii, John A. Burns School of Medicine,} 1951 East-West Road, Honolulu, HI 96822, USA.

The question of how individuals come to know that they are male or female has gone through several cycles with either nature or nurture given the nod as to supremacy. From the 1950 s to the 1990 s the greatest input was attributed to environmental forces, particularly upbringing, as being the most crucial influence in teaching the child it is either a boy or a girl destined to grow to be a man or woman. The later half of the decade of the 1990s, however, has dramatically shifted the thinking to give greater weight to intrinsic biological forces as being deterministic.

One of the most important cases in this debate has been that of the socalled John/Joan twin. Here was an XY individual raised as a girl from about a year in age, sans penis, scrotum and gonads and with preliminary surgery to fashion a vulva. From the age of 12 the twin was given estrogen medication to foster female pubertal development. Despite this treatment the twin came to identify as a male and eventually, at the age of 14 , rejected his living as a girl. This case will be discussed. Also to be discussed are other cases where twins in particular, but siblings and peers in general, are seen as important factors in how individuals come to recognize their biological sex and the social gender that best suits them. A mechanism, incorporating both intrinsic and extrinsic factors attempts to explain how this comes about.

\section{S}

\section{GENE-ENVIRONMENT INTERACTION \\ IN ALCOHOL USE AND ABUSE: DATA FROM FINNISH TWIN STUDIES}

Danielle M. Dick, Richard J.Viken, Jaakko Kaprio, Lea Pulkkinen, \& Richard J. Rose

Correspondence address: Indiana University, Dept. of Psychology,

1101 E. 10th St., Bloomington, IN 47405

Behavior genetic studies were heavily criticized in the past for their failure to include environmental measures; this criticism is no longer warranted, as genetically-informative studies are increasingly including environmental measures, and advances in biometrical modelling allow for the incorporation of specific environmental variables. With data from two population-based studies of Finnish twin adolescents, we have been exploring the effect of a variety of environmental influences on alcohol use/abuse and related phenotypes, and the interaction of these environments with genetic influences. In successive analyses, we illustrate the impact of various environmental factors, including socio-regional and community-level influences, home environment, and peer influences. Each of these environmental variables also illustrates a different way that the environment can interact with genetic factors. We document the presence of community-level environmental influences, and demonstrate socio-regional moderation of influences on alcohol use, with the importance of genetic and environmental influences varying more than five-fold between environmental extremes. Parental monitoring and home atmosphere contribute additively to adolescent behavior problems, while peers' alcohol use exhibits a more complex, interactive relationship with self-reported alcohol use. Thus, in a series of quantitative genetic analyses, we explore various environments involved in alcohol use and related phenotypes, and the manner in which these environments act and interact with genetic predispositions.

\section{P

TWIN STUDY OF MAMMOGRAPHIC BREAST
DENSITY FOR AGE AS A RISK FACTOR
FOR BREAST CANCER

Gillian S. Dite, Norman F. Boyd, Margaret R.E. McCredie, Dallas English,

Graham G. Giles, John L. Hopper

Correspondence address: Centre for Genetic Epidemiology, Carlton, Vic 3053, Australia

The proportion of dense breast tissue for a woman's age (breast density for age) has been found to be a strong risk factor for breast cancer by prospective case-control studies that matched for age nested within cohorts of women attending mammographic screening programs. We have digitised mammograms from both members of 617 female pairs of Australian twins aged 40 to 75 years (349 MZ, 268 DZ), and used a computerised algorithm to quantify breast density. We also collected information on demographic and potential determinants of breast density by an interviewer-administered questionnaire. Breast density was independent of age up to 50 years, but decreased in the next decades. The correlation in age-adjusted logit breast density was $0.67($ s.e. $=0.05)$ in $\mathrm{MZ}$ pairs and 0.27 (s.e. $=0.06$ ) in $\mathrm{DZ}$ pairs. Under the usual assumptions of the classic twin model, this would suggest that genetic factors explain the majority of variation in this risk factor for breast cancer. Given the inter-quartile risk of disease associated with age-adjusted breast density is 3- to 5-fold, this would translate into an increased risk of breast cancer due to having an affected twin of 1.2-fold within MZ pairs, and 1.1-fold within DZ pairs. That is, breast density for age could explain about $10-20 \%$ of familial aggregation of the disease on a population basis, similar to the proportion attributed to mutations in BRCA1 and BRCA2. Within-pair analyses that naturally match for age showed a strong association of breast density with weight, and a clear linear association with number of live births. Bivariate analyses of cross-twin correlations suggested that about half the within-person correlation between breast density for age and weight could be due to genes that influence variation in both traits.

\section{S MULTIPLE BIRTH CHILDREN'S LANGUAGE AND LEARNING ENVIRONMENTS}

\section{Barbara Dodd and Sandra McMahon}

Correspondence address: Department of Speech, University of Newcastle, UK

Studies of the speech and language acquisition of multiple birth children (MBC) have most often been used to estimate the contribution of genetic factors to development by comparing monozygotic and dizygotic siblings. There is, however, another way of using the evidence. Rearing more than one child of the same age in the same family, allows observation of the influence of specific language learning environments. The data suggest that there are differences between twins' and triplets' speech and language acquisition in terms of the aspects of language that are either advantaged or disadvantaged. The twin situation seems to enhance language use but place phonological acquisition at risk for disordered development, with consequent implications for literacy. In contrast, triplets have particular and persistent difficulty with pragmatic aspects 
of language, but early delays in expressive language structure are transient. Data from cross sectional and longitudinal case studies will be reported. Longitudinal data from one set of quintuplets suggest some specific difficulty in the development of receptive vocabulary. The results are interpreted in terms of the special language learning environments created by different MBC set sizes.

\section{P EXTRA RISK OF TRANSFERRING 3 EMBRYOS}

Michael Dooley, Anne Jones, Nicola Monks, Anthony Price, Jo Rice, Fran Rook Correspondence address: Fertility Unit, Winterbourne Hospital, Herringston Road, Dorchester, Dorset DT12DR

At the Fertility Centre our policy is to transfer 2 embryos. Our rate for elective transfer of 2 embryos is $67 \%$ compared with a national rate of $48.6 \%$. In deciding when to replace 3 embryos we use an individualised approach taking into consideration the patient's age, number of embryos available, number of unsuccessful attempts, whether the cycle is frozen or fresh and quality of the embryos. It is important prior to any procedure that full counselling, including risk implication, occurs.

Mr \& Mrs JL (born 1972) presented in 1997 with a 3-year history of unexplained primary subfertility as a couple. They were treated in several units with cycles of Clomiphene stimulation, one IUI with superovulation, with 2 good follicles, and one IVF attempt with 16 oocytes, 15 embryos and an unsuccessful 2 embryo transfer. They went on to a frozen embryo transfer with 6 embryos being thawed and 3 replaced, 2 at an 8 cell stage and one at a 4 cell stage. A pregnancy test was positive 4 weeks later and at 7 weeks gestation 4 sacs were seen in the uterine cavity. Additional counselling took place and a second opinion given re selective reduction. After nuchal translucency scanning and a chorionic villi sampling on one fetus the couple decided to continue with the pregnancy. This case demonstrates the need to fully inform couples not only about the potential risks of continuation of all the embryos replaced but also the risk of monozygotic twinning of the embryos.

\section{S WHAT WOULD IT MEAN TO THINK OF CONJOINED TWINS AS INDIVIDUALS? ETHICAL PROBLEMS IN THE MANAGEMENT OF CONJOINED TWINNING}

\section{Alice Domurat Dreger \& Lyman Briggs}

Correspondence address: Holmes Hall E-34, Michigan State University, East Lansing, MI 48825-1107, U.S.A.

Not unlike twins born separate, conjoined twins are subjected to singleton's misunderstandings and mythologies about the relationships between the twins' anatomies and their identities. Today singletons, especially surgeons, often presume that conjoined twins are "born to be separated." Indeed, surgery to separate twins (like surgery to assign sex in intersexed newborns) is called "reconstructive," as if the surgery were merely restoring the children to a state they once enjoyed. In fact, the surgery actively attempts to "normalize" the children, that is, normalize them according to the norms of singletons. Singletons see these surgeries as absolutely necessary, even when they leave previously able-bodied children disabled, potentially fertile children infertile, and, in the case of "sacrifice" surgeries, conscious children intentionally marked for death on the operating table. This paper explores the politics of individuality, the rhetoric of individual rights, and what it would mean to see conjoinment as part of what makes conjoined twins individuals.

\section{S}

\section{GENETICS OF BEHAVIOURAL DEVELOPMENT} - NEW QUESTIONS AND NEW MODELS

\section{Lindon Eaves}

Correspondence address: Virginia Institute of Psychiatric and Behavioral Genetics. Richmond VA 23298

The last quarter century has seen a growing investment in longitudinal twin studies that have been followed over the years in meetings of the ICTS. The typical approach to the analysis of time-series data in twins has been the linear structural ("Lisrel") model. Originally, such models were applied with a strong theory of the underlying mechanism of development in mind, but increasingly they have served a merely descriptive purpose that gives little new theoretical insight beyond the demonstration the genetic factors do/do not change with age. Many developmental mechanisms do not fit readily into the "Lisrel" framework. For example, the approach does not capture mechanisms in which the expression of genetic effects depends on passing a developmental milestone, the timing of which is itself subject to genetic (or environmental) variation, nor models in which there is simultaneous GxE interaction and correlation. Some alternative models of development are discussed and their application is examined using Markov Chain Monte-Carlo methods.

\section{GENETIC AND ENVIRONMENTAL INFLUENCES ON INDIVIDUAL DIFFERENCES IN DEVELOPMENT OF COGNITIVE STYLES IN CHILDREN FROM} 6 TO 16 YEARS

Marina Egorova \& Nadejda Zyrianova

Correspondence address: Psychological Institute of RAE, Mokhovaya st, 9 " V”, 103009, Moscow, Russia

The aim of this study is to analyze the impact of genetic and environmental factors on two cognitive styles - field dependence-independence (FD) and impulsivity-reflexivity (I-R). The sample consisted of 94 pairs of MZ and DZ twins. Subjects were tested five times: at 6 years (the last pre-school year), 7 years (the beginning of school education), 10 years (transition to middle school), 13 years and 16 years. This study is a part of Moscow longitudinal study of twins, started in 1989.

Instruments. Embedded Figures Test and Matching Familiar Figures Test, WISC and WAIS. The results showed rather stable role of FD in the structure of cognitive abilities: correlational analysis and analysis of variation (ANOVA) indicated that IQ was connected with FD, but not with I-R. Heritability of IQ appears to increase from 6 to 16 years. Genetic influences on FD are moderate and are growing from 6 to 16 ages. Heritability of I-R is low at 6 and 7 years and is growing from 10 to 16 years. Age-to-age genetic correlations between IQ and FD and I-R appear to be nonlinear, demonstrate interactions among these traits during development and different mechanisms of regulation of FD and I-R.

\section{S UNDERSTANDING CANCER DEVELOPMENT BY USING TWIN STUDIES}

\section{Anders Ekbom}

Correspondence address: Unit of Clinical Epidemiology, Department of Medicine, Karolinska Hospital, SE-171 76, Stockholm, Sweden

The intra uterine hormonal milieu has been proposed to be of importance for the risk of cancer, especially breast cancer, in adults. Twin pregnancies are therefore of interest, as they are associated with high pregnancy hormone levels. However, longitudinal studies of twins have (with the exception of early onset of breast cancer) revealed that twins do not differ in cancer risk from that of singletons. These findings do not necessarily refute the hypothesis of importance of the intra uterine hormonal milieu. Twin pregnancies are characterized by short gestational age, leading to difficulties to assess the cumulative exposure to pregnancy hormones. By the use of the Swedish Twin Registry, Cancer Registry and almost complete hospital archives two studies have so far been undertaken in order to study perinatal exposures and breast cancer. In one, in samesexed twins, a within-pair comparison revealed that mean birth weight and ponderal index were significantly higher among cases compared to their co-twins without breast cancer. We also substantiated the importance of gestational age where those with long gestational age had a higher incidence of breast cancer compared to those with a lower. In a second approach, opposite-sexed twins were studied in order to assess the importance of birth weight. In this study, there was a 12 -fold increased risk of breast cancer in female twins with the highest birth weight compared with those with the lowest, indicating that oppositesexed twin pairs constitute a special biological domain of interest.

In conclusion, twin studies of breast cancer indicate that the intrauterine milieu is of importance for the risk of breast cancer.

\section{F TAMBA FINANCIAL NEEDS CAMPAIGN FOR FAMILIES OF TWO OR MORE CHILDREN BORN OF A SINGLE PREGNANCY}

Jane Ellison

Correspondence address: c/o Harnott House, 309 Chester Road, Little Sutton, Ellesmere Port, $L 661 Q Q$

Changes in the differential level of family allowances paid to elder children, as against later siblings, in the 1998 budget and the government's insistence that the needs of the family were high on their agenda sparked a petition of multiple birth families through Twins and Multiple Births Association - Tamba. Members contacted local MPs for their support. Edinburgh Twins Club managed a face to face interview with their MP, Alistair Darling the Minister for Social Services. A response of 4359 signatures was taken to the House of Commons and presented to parliament on 27th April 1999. No acknowledgement was received, except in Hansard - the daily report of parliamentary proceedings.

Undaunted, Tamba surveyed its membership to discover what significance the payment of benefits, and other financial support, if any, made upon multiple birth families. This resulted in an excellent response 800 families with more still arriving. The results have been roughly analysed and good data achieved to show how a multiple birth arrival impacts upon any family and the real financial burden it can present, especially for low income families. A multiple birth family's costs are considerably more significant than a family where children are born 
at different intervals. Capital outlay is very high if a change in living accommodation and transport are necessary. Equipment costs can be far more than double those for a single child, and day to day consumables must also be brought into the equation.

\section{P}

\section{DO GENETIC FACTORS CONTRIBUTE TO} VARIATION IN INSULIN AND GLUCOSE LEVELS?

Erbas B', Hopper JL', Kyvik KO, Poulsen P, Vaag A, Beck-Nielsen H. Correspondence address: 'Department of Public Health, University of Melbourne, 200 Berkeley St, Carlton, Victoria 3053, Australia.

Aim. To determine if genetic factors influence variation in plasma insulin and glucose levels in males and females.

Design. Plasma glucose and insulin levels were measured after a 12 hour fast, at 30 and 120 mins following oral glucose ingestion, in a populationbased sample of 303 same-sex MZ and DZ twin pairs aged 55 to 74 years from the Danish Twin Register. Body mass index (BMI) and waist-to-hip ratio (wh-ratio) were also measured. $\mathrm{MZ}$ and $\mathrm{DZ}$ correlations in $\log$ transformed measures, adjusting for age, BMI and wh-ratio, were estimated by maximum likelihood separately for each sex.

Results. At fasting, 30 and 120 mins, correlations in insulin levels were stable at $0.32,0.46$ and 0.48 in $\mathrm{MZ}$ males, but decreased from 0.26 to 0.11 to 0.02 in DZ males. Similarly, for females these correlations were $0.52,0.44$ and 0.44 in MZ pairs and decreased from 0.49 to 0.29 to 0.09 in DZ pairs. Standard errors were about 0.10. In both sexes, therefore, the $M Z$ and $D Z$ correlations were no different at fasting. By 120 mins they were different $(p<0.02)$, but the DZ correlations were small and no different from zero. For glucose levels, the male correlations were $0.10,0.37$ and 0.46 in $\mathrm{MZ}$ pairs, and $0.18,0.25$ and 0.20 in $\mathrm{DZ}$ pairs, only differing at 120 mins $(p<0.02)$. For females, they were $0.58,0.52$ and 0.39 , greater than $0.06,-0.02,0.02$ in DZ pairs (all $p<0.02$ ), but none of the latter differed from 0 .

Discussion. There was no evidence for genetic variation in fasting levels in either sex. Given stable MZ correlations, genetic factors may influence response to oral glucose, but would involve non-additive effects and gene-gene interactions.

\section{F SECULAR AND SPATIAL DIFFERENCES IN TWINNING RATES IN SWEDEN, 1750-1970}

AldurW. Eriksson \& Johan Fellman

Correspondence address: Folkhälsan Institute of Genetics, Population Genetics Unit, POB 211, FIN-0025, Helsinki, Finland. aldur.eriksson@folkhalsan.fi.

To elucidate the causes and mechanisms of twinning and higher multifetal maternities, we have taken advantage of the statistical source of Sweden, where whole population continuous statistics are the oldest available. Rates of multiple maternities were highest during the last three decades of the 18th century, even during the years of privation, when natality decreased by $20-30 \%$. These are the highest rates recorded for a whole nation, the twinning rate being over 17 per 1000, triplet rate over 3 per 10,000 and quadruplet rate almost 7 per million maternities.

From 1836 until 1855 the twinning rate in Sweden was only 13.6 per 1000 , although, showing great regional differences being around 20 per 1000 in the county of Gotland (an island) and around 12 per 1000 in the county of Älfsborg in western Sweden. The marked differences in the twinning rate between these two counties are not explained by differences in the age distribution of the mothers. In the second half of the 19th century the twinning rate in the countryside in the county of Stockholm was around 20 but in the city of Stockholm only about 14 per 1000 . The decreasing trend observable in many regions during the first part of the 20th century up to the 1970's resulted in convergence towards relatively similar levels of around 10-12 per 1000. In Sweden after the 1930 's there was a marked decrease in the twinning rate, which by the 1960 's had fallen to only about half of what it had been two centuries earlier. The corresponding rates for triplets and quadruplet maternities were only about 25 per cent.

\section{P ASTHMA AND RESPIRATORY SYMPTOMS IN ITALIAN SCHOOLCHILDREN TWINS A STRUCTURAL EQUATION MODELLING APPROACH}

Corrado Fagnani, Rodolfo Cotichini, M.Grazia Calvani, Carla Rossi, M. Antonia Stazi and the SIDRIA group

Correspondence address: Istituto Superiore di Sanità, Rome, Italy

The Italian database of the ISAAC Project (International Studies on Asthma and Allergies in Childhood), the SIDRIA collaborative study, was reanalysed in order to estimate the life-time prevalence and the concordance of asthma and rinithis in the enrolled twin pairs. Among the 18,737 children $6-7$ years old and the 21,846 adolescents $13-14$ years old entered into the study, we were able to trace $393(2.2 \%)$ and $330(2.0 \%)$ twins respectively. The analysis has only been performed in 286 twin pairs distinguished between 202 same-sex (SS) pairs and 84 different-sex (DS) pairs. Information on zigosity was not available at this stage of the analysis. Based on the interviews to the parents the prevalence of asthma was $8.3 \%$ in twins versus $8.9 \%$ in singletons, and $7.9 \%$ in twins versus $10.4 \%$ in singletons for rinithis. The probandwise concordance was respectively 0.47 for same-sex pairs and 0.13 for different-sex pairs in asthma, 0.30 and 0.18 in rinithis. A structural equation model was fitted separately to same-sex and different-sex pairs. Variables considered in the model were: history of asthma and rinithis in parents, smoking in parents, early respiratory infections, number of siblings, pet animals, road traffic, socio-economic status. Based on genetic modeling, inherited genetic factors accounted for $11 \%$ in SS and for $3 \%$ in DS pairs of the liability in the inter-individual variation in the risk of overall asthma, while non genetic factors shared by twins accounted for $82 \%$ in SS and $71 \%$ in DS pairs and unique environmental factors for $7 \%$ in SS and $25 \%$ in DS pair.

\section{F}

TWINS - THE PARTICULAR ISSUES
FOR CHILDREN BORN AS A RESULT
OF DONOR-ASSISTED CONCEPTION

Julia Feast

Correspondence address: 49 Chillerton Road, London SW17 9BE (jmf@ childsoc.org.uk)

Approximately 7,500 children are born each year as a result of all forms of licensed fertility treatment in the UK. Of these, 2,500 are born following treatments involving donated gametes and embryos. In 1998/9, 75 live multiple birth sets were born resulting in 146 babies in all. For many of these children, the facts surrounding their conception and genetic make up will remain secret. Unlike adopted children, those born as a result of donated gametes have no rights of access to information about their genetic parent(s). We have learned from adopted people how crucially important it is to have accurate and detailed information about their origins if they are to make sense of themselves. Knowing who we are and where we come from and what makes us tick helps build a strong sense of self and identity.

Parents of twins are informed about the importance of treating their children as individuals and not as a pair or as one unit, so that each child can develop his or her own unique identity and personality. Parenting twins (or higher multiples) resulting from donor-assisted conception will therefore bring further additional tasks to the nurturing and child-rearing process, as often the genetic make-up of one or both parents is unknown. This presentation will therefore explore the particular issues and dilemmas for parents of multiples following donor-assisted conception, and look at ways to help them enhance their children's unique identities and personalities.

\section{P IMPACT OF MAGNETIC RESONANCE IMAGING OF THE FETAL BRAIN IN TWIN PREGNANCIES}

Vickie A Feldstein, Erin M Simon, Geoffrey A Machin, Nathaniel A Chuang, Roman Sydorak, Jody Farrell, Craig T Albanese, Michael R Harrison Correspondence address: Department of Radiology, Box 0628, University of California, San Francisco, CA USA 94143-0628

Magnetic resonance (MR) imaging enhances detection of fetal brain abnormalities which may be occult by ultrasound (US). There are high risks of brain injury in survivors of complicated twin pregnancies. We investigated whether fetal MR imaging provided additional information and how this affected management. Thirty-four pregnant women were studied. Gestational age at time of MR exam ranged from 18 to 31 weeks. Thirty-eight MR exams were performed on 60 living fetuses, targeting intracranial anatomy. Indications included twin transfusion syndrome(19), previous intervention(6), anomaly by US(5), and co-twin demise(5). Using commercially available equipment, modern ultrafast sequences were performed. T1- and T2-weighted MR images were acquired.

Compared with US, MR findings differed significantly in 9 cases and minor differences were shown in 12 . In 6 , a significant change in management was prompted based on MR findings. In 14, there was no change in management but increased diagnostic confidence was achieved. This included 4 evaluated prior to therapeutic procedure, 5 following co-twin demise, and 3 following intervention. In complicated twin pregnancies, MR imaging can be used to supplement or confirm US findings and may directly affect management.

\section{P ON THE STANDARDISATION OF THE TWINNING RATE}

Johan Fellman \& AldurW. Eriksson

Correspondence address: Folkhälsan Institute of Genetics, Population Genetics Unit, Helsinki, Finland, POB 211, FIN-00251 Helsinki,Finland. johan.fellman@shh.fi

In many studies the twinning rate has been standardised according to maternal age. The direct standardisation method requires highly informative data for the target population. The indirect standardisation method is used when the data for the target population are not informative enough or when the target population is so small that the age-specific 
twinning rates are subject to large random fluctuations that render the estimates inaccurate result. The only data that the indirect method requires are the age distribution of the general maternities and the total twinning rate. We earlier introduced an alternative technique for standardisation of the twinning rate, which required even less data about the target population, only the total twinning rate and the maternal mean age. In this study we present the traditional indirect and direct standardisation methods based on both maternal age and parity. Furthermore, we propose a new direct standardisation method and develop our standardisation methods so that they take into account both maternal age and parity. We have applied these standardisation methods to data for Finland, 19531964. The effect of maternal age is similar irrespective of the standardisation method but the effect of parity is strongest with the direct methods. This may be a consequence of the fact that parity strongly increases the twinning rates of unmarried mothers, probably due to their higher reproductive capacity. There are clear differences in the distribution of maternal age and parity between married and unmarried mothers and, consequently, the standardisation according to both maternal age and parity gives reliable results.

\section{S TWO'S COMPANY, THREE'S A CROWD}

Nicholas Fisk

Abstract not available.

\section{P TAMBA COMMUNICATIONS REVIEW}

Helen Forbes \& Judi Linney

Correspondence address: 1, Cardwells Keep, Guildford, Surrey, GU2 9PD

In 1998 the Twins \& Multiple Births Association (Tamba) undertook the first stage of a Communications Review, as part of an overall restruc ture. Direct communications to members - seen by many as the 'face' of Tamba and a source of information and support to parents - were Twins, Triplets \& More a magazine for members and Tamba Today, a newsletter, both published three times a year. A postal questionnaire, comprising both closed and open-ended questions, was returned by 310 members. Popular articles were those on child development, parents experiences, letters and information about older multiples. Respondents also identified research findings and twins in school as subjects they would like to read about. The internal business of Tamba was not popular and adverts and offers were criticised as irrelevant and out of date. Key Tamba personnel identified the need for an editorial team and more structure to the magazine to avoid repetition. The review recommended the merger of the two publications, with a Tamba news page in each issue to replace Tamba Today, and regular pages for the specialist support groups. A working party led by Helen Forbes was set up to implement the recommendations. These included the arrangement of a new, more flexible printing timetable, the establishment of a 5-strong editorial team and a much more critical editorial line. A two-year forward plan for contents was mapped out. The first issue of the relaunched magazine appeared in July 1999. This successful process of review will be illustrated on a poster by sample covers and pages from the magazine and the original questionnaire.

\section{$081 P$}

\section{THE HERITABILITY OF HAND GRIP STRENGTH - A STUDY OF 1624 DANISH TWIN PAIRS AGED 45-96}

Henrik Frederiksen, David Gaist,Axel Skytthe, James W.Vaupel, Matt McGue \& Kaare Christensen

Correspondence address: The Danish Twin Register, University of Southern Denmark

In gerontology it is a prevailing assumption that the accumulation of environmental exposures during a long life determines health and physical abilities at older ages. Evolutionary biologists have, on the other hand, argued that less selective pressure against deleterious mutations first expressed late in life compared to mutations expressed early in life predicts an increase in genetic variance among the oldest individuals. Findings from a recent study have supported the latter hypothesis with a substantial genetic influence on self-reported functional abilities in 75+ year old twins that increased among the oldest. A possible explanation of this could also be a ceiling effect of the used instrument in the younger old. We therefore used an objective measure of strength: Maximum Hand Grip Strength, which is a good indicator of overall physical strength. Furthermore grip strength declines linearly from mid 40's to 90 's. The study subjects were 1624 Danish twin pairs aged 45 to 96 . Hand grip strength was measured three times with each hand and the maximum value was used for further analysis. The MZ and DZ correlations of age adjusted grip strength were around 0.5 and 0.2 respectively for both males and females and were quite stable in all age groups. The heritability was overall roughly $50 \%$ in all age groups. In conclusion, genetic factors account for a substantial proportion of the variation in hand grip strength and are of a comparable size in middle aged, elderly and the oldest old.

\section{J. Gadzinowski,J. Mazela.}

Correspondence address: Department of Neonatology, University of Medical Sciences in Pozna?, Poland.

The aim was to review the literature for strong clinical evidence on standards of neonatal care for multiples. Multiple births represent $2.9 \%$ of the population.

Methods. The literature on neonatal care was surveyed focusing on 4 issues: prenatal steroids, growth charts for multiples, co-bedding and childhood development.

Results: 1) There are no neonatal or infant growth charts for multiples, although there are fetal growth charts for multiples which shows significant shifts from the ones for singletons. 2) Despite few publications on co-bedding twins, there is strong evidence that co-bedding helps thermoregulation, feeding and sleeping patterns and developing circadian rhythmycity. 3) Results and opinions differ on the use of higher doses of steroids in multiple pregnancies. Nevertheless according to the ACOG the doses used should be these same as for singletons. 4) The development and care of multiples is different to singletons due to LBW. These children have more disability, illness, school absenteeism, limitation of activities and hospitalizations. On average, the birth data are: $3358 \mathrm{~g}$ at $39.3 \mathrm{wk}, 2500 \mathrm{~g}$ at $36.2 \mathrm{wk}$ and $1698 \mathrm{~g}$ at $32.2 \mathrm{wk}$ for singletons, twins, and triplets respectively. For these reasons, NEC is 4 times higher in twins than in singletons, PDA 7 times, sepsis 2.4 times and IVH 20.2 times. Admissions to the NICU is 2 times higher for twins and 30 times higher for triplets. Neonatal death is 4.3 times higher for twins, and 7.9 times higher for triplets than singletons. Cerebral palsy incidence is 2.4/1000 in singletons, 6.7-12.6 in twins and 2.8-44.8 in triplets.

Conclusions and Speculation. Results indicate that more clinical attention should be given to this fast growing population of high risk babies, and not only to high risk pregnancies.

083F HIGHER MULTIPLES IN SCHOOL AND PRESCHOOL

\section{Diane Galloway}

Correspondence address: Tamba, Harnott House, 309 Chester Road, Little Sutton, Ellesmere Port, UK, CH66 1QQ

The aim of this survey was to collect information and points of view to help parents of higher multiples in making decisions about their children's school and pre-school choices. It could also be of value to teachers who may only meet triplets or higher multiples once or twice in their whole career. 135 questionnaires were completed, by parents whose children's ages ranged from 4 months to 19 years, with the majority aged under 5 years. Many of their concerns were similar to those of parents of twins but much more intense. Teachers need to be aware of the higher incidence of premature birth and special educational needs.

Voluntary groups for mothers with their babies and toddlers were greatly valued by the majority of parents who attended, for their own well-being as much as for their children's sake. They also found that other parents attending the sessions were a valuable source of information about the next stages in educating their children. The main reasons given for not attending were logistical problems in travelling to the group and concerns about keeping three or more children safe in a large room. Any help in overcoming these problems would be of long-term benefit to the families. Above the age of 3 years, the main priority was to provide for the needs of each individual child. At school, this means that each child must be easily identified and treated as an individual. At home, the need to give sufficient attention and help with homework was difficult for most parents to achieve.

\section{P}

\section{DIZYGOTIC TWINS}

IN A MONOCHORIONIC PLACENTA

Norman A Ginsberg', ', Svetlanna Rechitsky', Yuri Verlinsky',

Correspondence address: ${ }^{1}$ Illinois Masonic Medical Center, \& ${ }^{2}$ Northwestern University, 1520 Eastwood Highland Park, Illinois, 60035

Description of Case. A 35-year-old white female 611041concieved with clomaphine ovulation induction. At $52 / 7$ weeks of gestation a single intrauterine gestational ring was seen. At $62 / 7$ weeks, 2 fetuses and 2 yolk sacs were seen within a single chorionic membrane. At 8 weeks the two amniotic membranes were noted. CVS was performed at 11 weeks with two 46, XY karyotypes. Anomaly scan at 20 weeks was discordant for sex but otherwise normal. Amniocentesis revealed Twin A, to be 46, XY and the results of Twin B were 46, XX with FISH analysis revealed that $4 \%$ of the cells were $X Y$.

Proof of Diagnosis. DNA was extracted and 7 separate VNTRs were analyzes from Twin A, Twin B, and the mother. Analysis proved the twins were dizygotic without maternal contamination. Histology of the placenta confirmed that it was monochorionic and diamniotic. 
Relevance. Despite classic ultrasound findings and histology of the placenta, a monochoronic, diamnotic pregnancy may still be dizygotic. Prenatal diagnosis should be performed on each twin even when there is a high suspicion that the twins are monozygotic.

\section{P}

\section{GENETIC EPIDEMIOLOGY OF ALZHEIMER DISEASE IN ITALIAN TWINS}

Franco Giubilei, Micaela Sepe Monti, Simona Giampaoli, M.Fenicia Vescio, Luigi Ferrucci, Licia lacoviello, Marina Patriarca, Roberta Pacifici, Rodolfo Cotichini, Giovanni Ristori, Stefania Cannoni, Valeria Patriarca, Sonia Brescianini, M. Antonia Stazi

Correspondence address: Dept of Neurosciences, Univ. of Rome "La Sapienza", Italy

The Italian Ministry of Health promoted a comprehensive research program on Alzheimer disease, with one of the focuses being the interactions between genetic, environmental and behavioural risk factors. In this program, we have proposed a project with the intent to estimate the above-mentioned risk factors by using a twin population.

Our project will consist of two different approaches: first, in two Italian regions, Lazio and Tuscany, all cases of dementia in twins will be detected by matching the national twin registry to the national Alzheimer registry, established for monitoring the pharmacological treatment of the disease $(5,000$ patients affected by mild to moderate dementia are estimated). The concordance rates will be calculated according to zygosity. Second, in two areas of the previous regions, all members of the twin registry over 60 will be screened for dementia every two years, using a telephone interview. All subjects affected by dementia, enrolled with the two approaches, will be referred to a neurological clinic. Type of dementia and degree of neuro-psychological deficits will be assessed. All affected twins and their co-twins will undergo laboratory tests for genetic analysis and determination of markers for oxidative stress. Environmental and behavioural risk factors will also be recorded. The findings of these two approaches will be compared to estimate the relative efficiency of both approaches.

\section{P FETAL AND INFANT DEATH IN TWIN PREGNANCY:} CONSEQUENCE FOR THE SURVIVOR

\section{Svetlana V. Glinianaia*,Peter O.D. Pharoah, Chris Wright, Judith Rankin Correspondence address: Regional Maternity Survey Office, Dept. of Epidemiology and Public Health, School of Health Sciences, The Medical School, University of Newcastle Newcastle upon Tyne, NE2 4HH, UK}

Aim. To determine the long-term neurological morbidity for the surviving twin after a fetal or infant death of the co-twin.

Methods: Data on twin pregnancies delivered between 1981-92, with an antepartum or infant death, were identified from the Northern Perinata Mortality Survey. Information on the long-term neurological morbidity of infant survivors of a deceased co-twin was obtained from a questionnaire completed by the community paediatrician and/or general practitioner.

Results. There were 111 traceable children who survived infancy after a fetal death of a co-twin (group I) and 142 from live born twin pairs in which one twin died in infancy (group II). Response rates were $87 \%$ and $92 \%$ respectively. The cerebral palsy $(\mathrm{CP})$ prevalence in group I was 93 (95\% CI 43-169) per 1000 infant survivors (9/97). CP cases were more common in like-sexed (LS) pairs $(8 / 70)$ with a prevalence of 114 (95\% CI 51-213) per 1000 survivors compared with 45 (95\% CI 1-228) per 1000 in unlike-sexed (ULS) pairs (1/22). In group II, the CP prevalence was 154 (95\% CI 84-223) per 1000 infant survivors in LS pairs and 77 (9-251) per 1000 in ULS pairs. For twins born after 32 weeks of gestation, the CP rates for group I were twice as high compared with group II survivors. At an earlier gestation, the CP rates were higher for group II survivors.

Conclusion. The CP prevalence is substantially increased in the surviving twins after a fetal co-twin death in LS twin pregnancies compared with the general twin population. The CP prevalence is also high in the surviving twins in the infant-death/survivor group but mainly associated with prematurity.

\section{F CONGENITAL ABNORMALITIES IN TWINS, NORTHERN REGION OF ENGLAND 1998-1999}

Svetlana V. Glinianaia, Chris Wright*

On behalf of the Northern Congenital Abnormality Survey and the Multiple Pregnancy Register (MPR) Steering Groups.

Correspondence address: *Regional Maternity Survey Office, Dept. of Epidemiology and Public Health, University of Newcastle, Newcastle upon Tyne, NE2 $4 H H, U K$

Aim. To determine the prevalence of congenital anomalies in twins and to describe the types and clinical outcomes in anomaly-affected twin pregnancies.

Design. All twin pregnancies with a confirmed postnatal diagnosis of a major congenital abnormality, born during 1998-99 to mothers resi- dent in the former Northern Region of England, were identified from the population-based MPR. The MPR collects data on all multiple pregnancies within the region, whether they resulted in a spontaneous abortion, termination of pregnancy or birth.

Results. A total of 926 twin pregnancies delivered during 1998-1999 were notified. Sixty nine pregnancies were complicated by an anomaly, involving 83 individuals ( $4.5 \%$ of 1852 twins). The prevalence of anomalies was higher in monochorionic $(8.3 \%)$ than in dichorionic twins $(3.1 \%)(\mathrm{OR}=2.8,95 \%$ CI 1.6-4.9). Cardiovascular (17 individuals), renal (15) and chromosomal (11) anomalies were the most frequent. In five pregnancies, the anomalies were complications of monochorionic twinning: 4 sets of conjoined twins and 1 acardiac twin. Out of 69 twin pregnancies, 7 pregnancies (14 fetuses) were terminated, one was a selective reduction; of 62 liveborn twins, 52 (84\%) were alive at one year.

Discussion. The prospective MPR is a valuable source of data on multiple pregnancies, which can be used for the detailed investigation of congenital anomalies including follow-up studies.

\section{P MULTIPLE PREGNANCY REGISTER IN THE NORTH OF ENGLAND: 1998-99 RESULTS}

Svetlana V. Glinianaia, Chris Wright*, Judith Rankin, Marjorie Renwick Correspondence address: Regional Maternity Survey Office, Dept. of Epidemiology and Public Health, School of Health Sciences, The Medical School, University of Newcastle, Newcastle upon Tyne, NE2 4HH, UK

The twinning rate in the Northern Region of England has increased since 1991, with the perinatal mortality rate (PMR) 5.5 times that in singletons. A regional prospective Multiple Pregnancies Register (MPR) was established in January 1998 , with the aim of extending information on multiple pregnancies and to enable research into higher mortality and morbidity in twins. A multiple pregnancy is notified to the MPR from the first ultrasound examination irrespective of whether the pregnancy resulted in a spontaneous abortion, termination of pregnancy or birth.

A total of 926 twin pregnancies were recorded in the MPR during 1998-1999, giving a twinning rate of 14.8 per 1000 maternities. The twinning rate at birth was 13.0 per 1000 maternities $(809 / 62441)$ compared with 9.8 in 1990 and 12.0 in 1994. Twin pregnancies were detected before 13 weeks of gestation in $61 \%$ of cases. The PMR was 40.6 per 1000 twin births: 68.3 for monochorionic twins and 34.0 for dichorionic $(\mathrm{OR}=2.1,95 \%$ CI $1.1-3.8)$. Chorionicity was determined in $82 \%$ of twin maternities. Monochorionic twins were at a particular increased risk than dichorionic for a stillbirth: 54.0 vs 11.6 per 1000 $(\mathrm{OR}=4.8,95 \%$ CI $2.2-10.9)$. In $48(5.2 \%)$ twin pregnancies both fetuses died spontaneously in utero before 24 weeks of gestation. In $39(4.2 \%)$ pregnancies one twin was a livebirth after a fetal death of the co-twin before 24 weeks of gestation. The prospective MPR is not only important for observation of trends in multiple births rates and mortality, but also for randomised controlled trials and long-term follow-up studies.

\section{F CONCORDANCE FOR MAJOR DEPRESSIVE DISORDER CONTROLLING FOR BIRTH WEIGHT DIFFERENCES IN THE MISSOURI ADOLESCENT FEMALE TWIN STUDY}

\section{Anne L. Glowinski and Andrew C. Heath}

Correspondence address: Washington University School of Medicine, Psychiatry Dept, $40 \mathrm{~N}$. Kingshighway, Suite 1. St. Louis, MO 63108 USA

Introduction. Studies have reported an association of twin birth weight discordance (BWD) and discordance for variables such as fetal biometric parameters. We were interested in also examining the relationship between BWD and discordance for Major Depressive Disorder (MDD) in an adolescent twin sample.

Method. We analyzed data from 3584 Missouri female adolescent twins (MOAFTS) including 55\% MZ and 45\% DZ twins. The subjects were interviewed with the polydiagnostic Semi-Structured Assessment for the Genetics of Alcoholism (SSAGA) which includes a section on MDD. Parents gave detailed gestation information including twins' birth weights.

Results. Birth weight distributions in the MOAFTS were consistent with recent published norms. BWD was classified as $1(0-2 \%), 2$ (2 to $15 \%)$ or 3 (above $15 \%$ ). Controlling for zygosity, MDD discordance significantly correlated with BWD.

Discussion. With standard genetic analysis the variance for liability for MDD is explained by about half genetic and half unique environmental factors. Twin BWD could be a crude marker of discordance for unique in-utero environmental factors leading to greater discordance for MDD in adolescence. This is congruent with current concepts of brain development governed by epigenetic events within a genetic framework. Birth weight data from birth records will soon become available for replication of these results. 


\section{O9OF IS POST-TRAUMATIC STRESS A RISK FACTOR FOR CORONARY HEART DISEASE?}

Jack Goldberg, Viola Vaccarino, Mary Fischer, Seth Eisen, William True,

Michael Lyons, MaryEllen Vitek, William Henderson.

Correspondence address: VET Registry, PO Box 1389, Hines, IL, 60141 USA

Objective. Few studies have examined the long-term impact of post-traumatic stress (PTS) on the physical health of combat exposed veterans The purpose of the current investigation is to examine the association between symptoms of PTS and coronary heart disease (CHD).

Methods. A cohort of $\sim 4,700$ male twin pairs who served in the military during the Vietnam era where surveyed to obtain data on zygosity, PTS and CHD prevalence in 1986 and 1991. In 1999 incident cases of physician diagnosed CHD were obtained from a mailed follow-up.

Results. Unadjusted analysis revealed a pattern of increasing risk for CHD with increasing level of PTS ( $p_{\text {trend }}=0.001$ ). Compared to those in the lowest PTS symptom tertile, twins in the middle tertile had 1.4 times the risk of CHD (95\% CI 0.8-2.4) while those in the highest PTS tertile had 1.8 times the risk (95\% CI 1.0-3.2). The association between the PTS symptom index and CHD persisted after adjustment for sequential confounding factors. Simultaneous adjustment for major confounding factors greatly reduced the PTS-CHD association $\left(p_{\text {rend }}=0.31\right)$ and produced odds ratios of 1.2 and 1.1 for the middle and high tertiles.

Conclusions. These findings represent the first cohort investigation of PTS and CHD in Vietnam era veterans. It remains unclear if the association between PTS and CHD is a reflection of confounding or whether PTS indirectly increases CHD through known CHD risk factors.

\section{S DISABILITY IN TWINS}

\section{Paul Gringras}

Correspondence address: Multiple Births Foundation, Queen Charlotte's Hospital,

London 12 OHS

The term 'disability' encompasses a wide spectrum of neurodevelopmental disorders from those with a predominately motor component such as cerebral palsy, to those which affect cognition and acquisition of scholastic skills such as dyslexia. Whilst in many of these areas much has been learnt from twin studies, far less has been learnt about the twins themselves. Twin pregnancies account for about $2 \%$ of live births but result in a disproportionately high number of adverse outcomes.

This talk will discuss:

1. Cerebral Palsy in twins, importance of chorionicity and the "vanishing twin' hypothesis.

2. The role of newer non-invasive intrauterine cerebral studies in understanding underlying mechanisms of brain damage in twins.

3. ADHD and other specific learning difficulties such as dyslexia the lack of evidence that each exists as discrete categories, and importance of early language development in their aetiology.

4. Twin studies showing increased rates of language delay, ADHD and specific learning difficulties

5. The implication from such findings, if replicated, to cast doubt on the validity of generalising from twin disability studies to the general population, and the exiting prospect of early intervention.

\section{P MANAGEMENT OF A DICHORIONIC TWIN DISCOR- DANT FOR INTRAUTERINE GROWTH RESTRICTION}

Ahmet Gul, Halil Aslan, Ceyhun Numanoglu,Ahmet Gulkılık

Correspondence address: SSK Bakirkoy Dogumevi, Bakirkoy, Istanbul, Turkey.

A primigravid woman was referred to maternal-fetal unit of SSK Bakirkoy Maternity Hospital with the diagnosis of 32 weeks twin gestation discordant for intrauterine growth restriction (IUGR) in October15, 2000. BPD, FL, AC of larger twin were 32, 31, 32 weeks with norma amniotic fluid and of smaller twin were 29, 29, 27weeks with oligohydramnios respectively. Her blood pressure, hemogram, blood biochemistry, urine analysis and bilateral uterine artery doppler velocimetry were normal. Admission non-stress test (NST) of both fetuses was reactive and with these finding $s$ the diagnosis of twin discordant for IUGR was made. Two days after admission, NST of smaller twin became non-reactive with spontanous fetal heart rate deceleration without uterine contraction and decreased beat to beat variability. We performed an amniocentesis to larger twin for ensuring fetal lung maturity, presuming that smaller twin has mature lung. After ensuring lung maturity(phosphatidylglycerol $110 \mathrm{mg} / \mathrm{L}$ ), we delivered the twins abdominally with $2050 \mathrm{gr}$ and $1400 \mathrm{gr}, 7 / 9,5 / 9$ apgar scores in one and five minutes. The hematocrit of larger twin was $42 \%$, blood group $\mathrm{B}-\mathrm{Rh}(+)$ and smaller twin had a hematocrit of $65 \%$, blood group $\mathrm{O}-\mathrm{Rh}(+)$ Three months after delivery both twins were healthy. In conclusion, as in our case, twin with growth discrepancy and one with fetal distress, performing amniocentesis only to the larger twin for ensuring lung maturity may help in management and prevent iatrogenic premature delivery for larger twin and fetal death for the smaller one.

\section{STABILITY AND CHANGE IN PERCEIVED AUTONOMY DURING EARLY ADOLESCENCE: CITY VERSUS KIBBUTZ}

Ruth Guttman, Siv Fischbein and Nitzan Goldschmidt

Correspondence address: Department of Psychology, The Hebrew University of Jerusalem, 91905 Jerusalem, Israel

The study compares perceptions of 9- to 13-year-old twins of restrictions imposed on them by parents and teachers in two rearing environments: Jerusalem and kibbutz. Kibbutz children spend much of their time in special children's houses, share teachers, caregivers, food and resources during a significant part of the day. Jerusalem children, in comparison, spend the better part of the day with parents and sibs.

Each subject rated, three years in succession, levels of autonomy / control experienced at home and in school. Among the questions asked were the extent to which father, mother and teacher told the twins what to do, regulated choices at home and in school, imposed regular hours for certain activities. Kibbutz and Jerusalem twins did not differ in their assessment of restrictions imposed on them by either parent. No changes in ratings of parents were found between ages nine to thirteen, either in Jerusalem or in the kibbutz. Teachers, however, were rated as more permissive with increasing age. At all age levels kibbutz twins reported less control by their teachers than did city twins. These findings reflect the special role of the kibbutz teacher in early adolescents' intellectual and social development. A comparison of MZ and DZ within-pair correlations suggests a genetic contribution to the twins' perception of parental and teachers' permissiveness/restrictiveness. Both MZ and DZ intraclass correlations were larger at age thirteen than at age ten, but the MZ / DZ ratio remained the same from late childhood through early adolescence.

094P

\section{HELPING HELPERS TO HELP - GUIDELINES FOR PROFESSIONALS} WORKING WITH MULTIPLE BIRTH FAMILIES

\section{Faith Hallett, Jane Denton and Elizabeth Bryan}

Correspondence address: Multiple Births Foundation, Queen Charlotte's

and Chelsea Hospital, Du Cane Rd, LondonW12OHS mbf@ic.ac.uk

Since it started in 1988, the Multiple Births Foundation (MBF) has been approached by many parents of multiples with problems, often longstanding, that could have been resolved much sooner had their professional carers appreciated their special needs and known how to respond. A recent survey of the Telephone Advisory Service showed $40 \%$ of calls concerned routine management problems such as sleep, feeding, toilet training and biting. Similarly, at the Twins Clinic, many referrals are related to communication, behaviou $\mathrm{r}$ and discipline problems. Because it is not available locally, couples often seek information to prepare them for multiple parenthood through the MBF prenatal meetings.

A comprehensive series of Guidelines for Professionals has therefore been published by the MBF (1997-2001) and part funded by the European Union. These titles, Facts about Multiple Births; Multiple Pregnancy; Bereavement: Special Needs; The First Five Years and Beyond, are aimed at a wide spectrum of professionals from infertility specialists to paediatricians, midwives, community health workers, social workers and teachers. The books, which concentrate on management and social issues specific to multiple birth families, are divided into sections. Each of these provides a background to the topic (with references to research) and recommendations on how best to respond. Although the Guidelines relate to the health care in the UK, they are designed for the easy translation and adaptation for individual countries.

\section{F ICELAND: SETTING UP AN ANTENATAL CLASS FOR PARENTS EXPECTING TWINS}

Karitas Halldors dottir, Unnur B. Fridriksdottir

Correspondence address: Skolagerdi 63, 200 Kopavogur, Iceland

As midwifery students part of our education involves setting up and running an antenatal class. Since we are both mothers of twins we decided to run a class for parents expecting twins. This has never been done before in Iceland. The need for a specially designed class for these parents seems to be great, as the response we got, from parents, was beyond our expectations but health care providers were more doubtful. The class was twice a week for a total four evenings and our small population gave us the opportunity to include pregnancy care, giving birth and parenthood in our antenatal class, as well as a visit to the delivery ward and the neonatal intensive care unit. The chairman of the Icelandic twin club came and introduced the twin club. 

AND EXPOSURE IN CALIFORNIA TWINS

Ann S. Hamilton, Myles G. Cockburn, Wendy Cozen, and Thomas M. Mack. Correspondence address: Keck School of Medicine at USC, 1441 Eastlake Ave. MC9175, Los Angeles, CA 90089, USA

The California Twin Cohort consists of a population-based cohort of twins born in California who have completed a risk factor questionnaire. Currently, nearly 30,000 pairs are represented in the cohort. Information on personal smoking as well an exposure to environmental tobacco smoke was obtained. Factors related to initiation and persistence of personal smoking and exposure to parental smoking will be discussed. Preliminary analyses of 22,000 pairs has shown that within all zygosity groups those with more than a high school education were half as likely to smoke as those with lower education and those whose parents smoked were $50-60 \%$ more likely to smoke than offspring of non-smoking parents. However, the influence of the co-twin's smoking habit was the most important factor with identical twins (both male and female) 13-16 times more likely to smoke if their twin did and fraternal twin 5 times more likely. Study was also made of the effect of the twins' parents smoking habits and the occurrence of selected congenital abnormalities including clubfoot, congenital heart problem, cerebral palsy, cleft lip, spina bifida, and strabismus (lazy eye). Odds ratios for maternal and paternal smoking were calculated as well as probandwise concordance rates. A significantly elevated OR for parental smoking and strabismus was found $(\mathrm{OR}=1.8,1.4-2.3)$ and probandwise concordance rates were higher among MZ than DZ twins suggesting the possibility of both an environmental and genetic role in the development of this condition.

\section{F}

\section{HOW REPRESENTATIVE IS THE WESTERN AUSTRALIAN TWIN REGISTER?}

Janice Hansen, Nicholas H. de Klerk, Maxine L. Croft, Phyllis D. Alessandri, Paul R. Burton

Correspondence address: TVW Telethon Institute for Child Health Research,

P.O. Box 855, West Perth, 6872, Australia.

Objectives. To assess the representativeness of the population-base d West Australian (WA) Twin Register.

Methods. The WA Twin Register comprises data on multiple births in WA from 1980-1992 inclusive. Families that could be traced were sent questionnaires to complete, to determine the genetic and environmental contributions (if any) linking passive smoking to childhood asthma and atopy. The representativeness of the Register was assessed by comparing outcomes and exposures in multiples on the Register with those in (i) multiples not on the register, and (ii) singletons born during the same period.

Results.

There was no difference between mothers participating in the study and those not participating in the study with respect to place of residence, age at first birth and race. Prevalence of doctor-diagnosed asthma in children of families on the Register was $28 \%$ compared with $29 \%$ in singletons born during the same period. Parents were more likely to respond if their multiples do not have cerebral palsy or a birth defect.

Conclusions. The WA Twin Register appears to be representative of the WA population with respect to childhood asthma and atopy. However factors that modulate response are still evident when comparing responders with non-responders, and the WA Twin Register may not be representative with respect to any outcome that which is likely to be modulated by such factors.

\section{P}

\section{FACTORS AFFECTING CONCORDANCE RATES FOR TYPE 1 DIABETES IN IDENTICAL TWINS}

Hawa MI, Redondo MJ, Tu L, Mackenzie T, Pyke DA, Eisenbarth GS, Hitman GA, Leslie RDG

Correspondence address: Department of Diabetes \& Metabolism, St Bartholomew's Hospital, UK

To determine the rate and factors which influence concordance in monozygotic (MZ) twin pairs discordant for type 1 diabetes, we studied non-diabetic MZ twins of patients with type 1 diabetes from Great Britain $(n=134)$ and the United States $(n=53)$. Discordance for disease between identical twins implies a role for non-genetically determined factors but could also be influenced by a decreased load of diabetes susceptibility genes. By survival analysis of the series from UK and USA ( $n=187$, median follow-up 17.7 years, range $0.01-40$ ), the combined probability of progression to diabetes was 39\% (SEM 7\%) at 40 years of discordance, with striking similarity between the two series. In twins of index cases diagnosed at 24 years or younger the probability of progressing to diabetes by 30 years discordance was $38 \%$, but only $6 \%$ for the remaining twins $(p=0.004)$. In non-diabetic MZ twins antibodies to glutamic acid decarboxylase or IA-2 were highly predictive of IDDM (positive predictive value $>86 \%$ ). Antibody isotypes tended to be restricted to the IgG1 subclass. Significantly more MZ than dizygotic twin pairs were concordant for the presence of antibody combinations $(33 \%$ vs $6 \% ; p<0.05)$ and the development of diabetes, (33\% vs $0 \% ; p<0.01)$. In $77 \mathrm{MZ}$ twin pairs (40 concordant and 37 discordant for Type 1 diabetes), the high risk HLA DQB $1 * 0201$ /DQB1*0302 or the Hph I insulin genotype was found more often in the concordant twins 38 of $40(95 \%)$ compared to discordant twins 25 of 37 $(68 \%)(p=0.02)$. Twins of diabetic patients from two different countries have remarkably similar rates of progression to diabetes with late progression to diabetes in a fraction. Progression to diabetes in nondiabetic MZ twins was related to age at diagnosis of the index twin, $\mathrm{MHC}$ and non-MHC gene load and the presence of antibodies.

\section{S BEHAVIOURAL DATA IN VOLUNTEER} AND POPULATION-BASED TWIN REGISTERS

David A Hay, Michael McStephen, Janice Hansen, Florence Levy,

Jillian Pearsall-Jones, and Erik Rasmussen

Correspondence address: School of Psychology, Curtin University, GPO Box U1987,

Perth,WA 6845, Australia

Apart from the very rare studies where all data are obtained from medical and other records, most twin registers are based on those who do volunteer or who are approached for participation after their twinship is identified from records. How comparable are data from different sources? WA is unique in that there are two overlapping groups

1. Twin families who agreed to participate after being approached in the population-based WATCH study (WA Twin Child Health Study). WATCH only began in 1997 and

2. Some WA families had previously agreed to participate in the volunteer Australian Twin Registry (ATR) which covers all states including WA and which has run for over 20 years.

This presentation focuses on three issues in childhood behavioural problems especially ADHD.

1. Few differences in reported behavioural problems between the volunteer ATR and the population-based WATCH study

2. Selective attrition in the ATR study of families over ten years of those at both extremes of the population

3. Comparability of data using the Missouri Adolescent Female Twin Study which produced very similar models of ADHD to ATR despite recruitment and assessment differences.

Conclusion. How twin registers are derived matters less than what exclusion criteria are used (16\% in the ATR sample), how behaviour is assessed and especially what behaviours are being studied. Is ADHD an especially robust measure? The need for benchmarks across all registers is emphasised.

\section{$100 \mathrm{~S}$ DEVELOPING THE MULTIPLES IN SCHOOL WEBSITE}

\section{David Hay ', Pat Preedy ${ }^{2}$}

Correspondence address: ${ }^{1}$ Curtin University, Western Australia; ${ }^{2}$ Knowle CE Primary School, Solihull, UK

Special challenges face parents and teachers of twins and higher multiples. Questions such as whether or not to separate multiples in school do not arise with singleborn children. There are many other unique dilemmas that can arise, for example, what if one twin but not the other is ready to start school or what to do if one is succeeding much better than the other(s)? Few professionals have good knowledge of the needs of multiples and their parents. This session introduces a new website designed for parents, teachers and the range of other professionals such as speech and language pathologists and educational psychologists that the multiple birth family is more likely to encounter.

With the growing number of multiples and especially higher order births, it is important that everyone has ready access to accurate and current information on the issues. Our extensive studies in Australia and the UK provide a sound empirical base for the material that is included. There are five main topics (Multiple Facts and Figures, Preschool, The School Years, Special Needs and Multiple Links). As well as resource material, each section has checklists, for example, on school readiness and on behaviour problems that form the basis for discussions about the needs of particular sets of multiples. There are also scenarios to generate debate in the forums. It is hoped that this website will be the basis for an ever expanding resource that will serve multiples, their parents and professionals. 


\section{$101 S$ MYTHS AND REALITY IN DECISIONS} ABOUT MULTIPLES IN SCHOOL

\section{David Hay ', Pat Preedy}

Correspondence address: ${ }^{1}$ Curtin University, Western Australia; ${ }^{2}$ Knowle CE Primary School, Solihull, UK

We review the literature about whether or not multiples should be in the same class. There is a major difference between literature and evidence. The first empirical study by Koch (1966) showed no evidence that separation was appropriate. Yet there have been many comments that multiples should be separated, based both on anecdotal (and biased) reporting and especially on the well-publicised cases of very dependent and dsyfunctional multiples, since this is the only information teachers are likely to encounter. It appears in news not science. In 1989 Australia had the first extensive survey of parents and teachers of multiples, followed with our comparable study of UK families several years later. There are remarkable similarities in the two surveys in terms of issues that should be considered, along with some health promotion differences in that Australian parents and teachers have long been exposed to the issue of speech delay in multiples. The most important issue is not that the same factors were identified in the two countries regarding separation but that there was a clear recognition that separation was not to be based on one issue but on many.

Our Multiples in School website emphasises best practice in what should be achieved for each multiple birth, but also what factors to identify in making the decision. It is only recently that such behavioura disorders as ADHD have been identified as more common in multiples and there are many issues about recognition and acceptance of the implication of these for multiples. We conclude with a difficult issue to what extent should families and professionals be sensitised to the needs of multiples or would this be stereotyping of possible problems?

\section{S THE PSYCHOSOCIAL IMPACT OF HAVING OR BEING A DISABLED TWIN}

\section{David A. Hay, Lisa Studman and Clare Roberts}

Correspondence address: School of Psychology, Curtin University, GPO Box U 1987,

Perth, 6845, Australia

Shere (1955) claimed it was worse to be the healthy cotwin rather than the twin with cerebral palsy. This emphasises that most studies focus on the prevalence of disability in multiple birth families, rather than the broader psychosocial impact on the disabled twin, the cotwin or the parents. But families respond differently to the stress of having one twin who is disabled and this is fundamental to early intervention with multiple birth families. Based on many years experience of multiple birth families, we introduce three issues:

1. Is it more difficult to accept that one twin is disabled-we discuss this in terms of a new WA program to help parents come to terms with disability in one child

2. How does the non-disabled twin cope and what are the pressures they experience?

3. Does this issue apply across all disabilities? There is a question that some problems such as speech or ADHD may be attributed to "just being a twin" or to gender differences "boys are like this"

We discuss two complementary models:

1. Taylor's (1983) model of Family adaptation to disability

2. The ABCX model of family stress that focusses intervention on what resources are needed or perceived to be needed, given differences in coping strategies

Conclusions. Given the higher rates of disability in multiples, it is vital to have such models and agency responses fit with the particular needs of multiple birth families.

\section{$103 S$ SECULAR CHANGE AND GENE-ENVIRONMENT INTERACTION EFFECTS IN ALCOHOLISM: RESULTS FROM THE YOUNG ADULT AUSTRALIAN TWIN COHORT}

A.C. Heath, K.K. Bucholz, E. Nelson, P.A.F. Madden, W.S. Slutske and N.G. Martin Correspondence address: Missouri Alcoholism Research Center, Washington University School of Medicine, St Louis MO, U.S.A

Despite secular and cross-cultural differences in rates of alcoholism, it has proved difficult to demonstrate differences in the heritability of alcoholism between different birth cohorts, or different societies. Examination of birth cohort differences within a society is complicated by factors such as differ ential mortality, and differential recall bias for different age-groups. Two cohorts of young Australian twins, born 1956-1964 ( $N=900$ twins), and $1964-1971$ ( $N=6265$ twins $)$ have completed diagnostic interview assessments at approximately the same age, allowing an analysis of cohort differences in drinking patterns and problems. In women, while no mean differences in consumption levels were observed, the more recent birth cohort showed greater frequency of heavy drinking, drinking to intoxication, and related problems such as blackouts and alcohol-related accidents. Lifetime histories of alcohol dependence were reported with much greater frequency by the more recent cohort (15\% for DSM-IV alcohol dependence) than by the older birth cohort ( $9 \%$ by less stringent DSM-IIIR criteria). Despite these differences, the estimated heritability of alcohol dependence differed minimally between the two cohorts, with a trend for lower heritability in the younger cohort $(50 \%)$ than in the older $(65 \%)$, contrary to our a priori expectation that reduced social prohibitions against drinking to intoxication by women would lead to evidence for stronger genetic influence in the younger cohort. (Supported by NIH grants AA11998, AA10249 \& AA07728)

\section{P DISCORDANCY AND CATCH-UP GROWTH IN TWINS}

Mary L. Hediger, Barbara Luke, Ruta Misiunas, Elaine Anderson

Correspondence address: University of Michigan, Ann Arbor, Michigan, USA

As part of an ongoing follow-up study of twins, 73 pairs (146 children) born between 1996-98 were measured for length (LT) or height (HT), weight (WT), and head circumference (HC). The purpose of this study was to evaluate whether intrapair differences present at birth persist through 3 -years age. Of the twins followed to date, 38 pairs were measured at 8 -months, 40 pairs at 18 -months, and 15 pairs at 3 years. The intrapair difference in WT at birth was $275 \mathrm{~g}$; the larger 2,529 $\pm 581 \mathrm{~g}$ (birthweight zscore, $-0.22 \pm 0.70$ SDU) and the smaller $2,244 \pm 583 \mathrm{~g}$ $(-0.86 \pm 0.67 \mathrm{SDU})$. At 8 -months there were significant differences in WT $(+0.50 \pm 0.73 \mathrm{SDU}, p<.01)$ and $\mathrm{HC}(+0.43 \pm 1.07 \mathrm{SDU}, p<.05)$, and a borderline difference in LT $(+0.30 \pm 1.06$ SDU $)$. The lighter twin was still lighter $(-0.30 \pm 1.17 \mathrm{SDU}$ vs $+0.08 \pm 1.30 \mathrm{SDU})$, but demonstrated more rapid catch-up growth compared to the heavier twin $(+0.63 \pm 1.20$ SDU vs $+0.35 \pm 1.29$ SDU, $p<.01)$. By 18 months, the differences in HC $(+0.28 \pm 1.28$ SDU $)$ and HT $(+0.10 \pm 0.80$ SDU) were much less, and only the difference in WT was still significant $(+0.38 \pm 0.87$ SDU, $p<.05)$. Likewise, at 3 years, the twin born heavier was still heavier $(+0.43 \pm 0.56 \mathrm{SDU}, p<.01)$, but there were no significant differences in HT, and almost complete catch-up in WT, with the twin heavier at birth averaging $+0.51 \pm 0.81$ SDU for WT and the twin lighter at birth $(+0.08$ \pm 0.97 SDU), both catching up about half an SDU.Thus, while relative intrapair differences, especially in WT, appear to persist through age 3 , there is significant catch-up, with the twin smaller at birth catching up more than the larger of the pair.

\section{P SERUM FERRITIN AND TWIN FETAL GROWTH}

\section{Mary L. Hediger, Barbara Luke, Clark Nugent}

Correspondence address: University of Michigan, Ann Arbor, Michigan, USA

Extremes in maternal serum ferritin levels have been implicated in poor fetal growth for singletons, but their diagnostic value in twin pregnancies is not known. This study evaluated the relationships between serum ferritin, maternal weight gain, and fetal growth in twin pregnancies. Serial measures of iron status, maternal weight gain, and outcomes were collected for 129 mothers of twins. Weight gain at 24 weeks (WG24) averaged $12.4 \pm 5.1 \mathrm{~kg}$, birthweight (BWT) $2,488 \pm 504 \mathrm{~g}$ at $35.5 \pm 2.3 \mathrm{wk}$. Adjusting for ethnicity and parity (mean $\pm \mathrm{SE})$, ferritin was $63.1 \pm$ $4.0 \mu \mathrm{g} / \mathrm{L}$ in the $1 \mathrm{st}$ trimester $(11 \mathrm{wk}), 34.6 \pm 3.0 \mu \mathrm{g} / \mathrm{L}$ in the $2 \mathrm{nd}(19 \mathrm{wk})$, $11.3 \pm 2.4 \mu \mathrm{g} / \mathrm{L}$ in the $3 \mathrm{rd}(29 \mathrm{wk})$, below the cutoff for iron deficiency $(12 \mu \mathrm{g} / \mathrm{L})$. After gestation, WG24 was the most significant predictor of BWT $(15.2 \pm 5.2 \mathrm{~g} / \mathrm{kg}$ weight gain, $p<.005)$; $3 \mathrm{rd}$ trimester ferritin levels were lower with higher WG24 $(-0.18 \pm 0.07 \mathrm{~kg} / \mu \mathrm{g} / \mathrm{L}, p=.01)$. In the $3 \mathrm{rd}$ trimester, ferritin levels were $16.1 \pm 1.5 \mu \mathrm{g} / \mathrm{L}$ and BWT $2,344 \pm 72 \mathrm{~g}$ for those with WG $24<9.1 \mathrm{~kg}$, vs serum ferritin of $9.8 \pm 1.2 \mu \mathrm{g} / \mathrm{L}$ and BWT of $2,581 \pm 55 \mathrm{~g}$ for those with WG24 $>15.5 \mathrm{~kg}(p<.05)$. In addition, BWT was lower $(p<.01)$ with serum ferritin levels $>14.25 \mu \mathrm{g} / \mathrm{L}(2,421 \pm$ $53 \mathrm{~g})$ vs $<7 \mu \mathrm{g} / \mathrm{L}(2,660 \pm 52 \mathrm{~g})$. These findings indicate that while most twin mothers may have 3rd trimester serum ferritin levels consistent with iron deficiency, unlike for singletons, this may be a clinical indicator of better fetal growth, as iron stores are utilized to support fetal growth. High levels, which may still be well below ferritin values found in the 3rd trimester for singletons with good outcomes, may be an indicator of growth restriction in twin pregnancies.

\section{S MOLECULAR ANALYSIS OF BREAST CANCER IN MZ TWINS}

Kari Hemminki and Asta Försti

Correspondence address: Biosciences at Novum, Karolinska Institute,

14157 Huddinge, Sweden.

A new approach has been tested in mapping of allelic imbalances in DNA of monozytic twins. Such allelic imbalances may be indicative of locations of tumour suppressor genes. Loss of heterozygosity $(\mathrm{LOH})$ analysis was carried out on microdissected tumour and normal tissue from concor- 
dant tumours of monozygotic twins. The method relies on recognition of genomic locations undergoing loss in both twins in a high proportion of the set of all twin pairs examined. Using breast cancer samples from 9 twin pairs, identified through the Swedish Twin and Cancer Registry, studies on 4 chromosomes have been carried out, each with some 15 microsatellite markers. Overall, only chromosome arms $16 \mathrm{q}$ and $17 \mathrm{p}$ showed evidence on a heritable effect - a tendency for both twins to lose the same allele. Some individual markers at chromosomes $13 \mathrm{q}$ $16 q, 17 p$ and $17 q$ displaye d heritable effects, some of these close to known tumour suppressor genes. In the course of these studies we analysed the samples for BRCA1 and BRCA 2 mutations. No BRCA1 mutations were found but 2 sequence variants of BRCA2 were identified. The efficiency of the MZ twin method depends on the overall rate of LOHs in tumour samples. Even though the overall rate is high, some $50 \%$, in breast cancer, only a few MZ pairs are needed to call a significant effect. The model is basically similar to the use of multiple cancers from the same individual. We believe that both the twin and the multiple cancer models will be useful for molecular studies of polygenic cancers The downside is that the identification and sample procurement from both of concordant MZ twins is not trivial.

\section{ASSISTED REPRODUCTION - COUNTING THE COST. ECONOMIC IMPLICATIONS OF MULTIPLE BIRTHS}

Jane Henderson and Stavros Petrou

Correspondence address: National Perinatal Epidemiology Unit, Institute of Healt Sciences, University of Oxford, Headington, Oxford OX3 $7 L F$, United Kingdom.

Although twins and high order multiple births account for a relatively small proportion of deliveries, they contribute disproportionately to the overall numbers of stillbirths, infant deaths and low birthweight babies. Children from multiple births, particularly those from triplet and higher order births, impose a greater burden on the health, education and social services, and on their parents and informal carers, than singletons. Moreover, multiple maternities also appear to be associated with higher rates of morbidity and mortality in the mother throughout pregnancy and childbirth. Consequently, questions have emerged about the economic implications of increasing multiple birth rates in many industrialised countries and the costs that can be attributed to the use of assisted conception and of drugs used for the medical management of sub-fertility. This presentation will review published and unpublishe d evidence regarding the economic implications of twins and higher order multiple births for the health services, for other sectors of the economy and for individuals. The presentation will discuss the assumptions upon which economic models are based and will also define a minimum set of methodological standards upon which future economic studies of multiple births should be based. In addition, the presentation will summarise the scope and scientific quality of existing evidence in order to identify gaps in our knowledge and to consider the future research agenda in this area.

\section{P ROMANDE ASSOCIATION OF HELP, EXCHANGES AND INFORMATION CONCERNING THE TWINS, TRIPLETS AND MORE}

Sabine Herbener

Correspondence address: Ch. de Ballalaz 18, CH-1820 MONTREUX

sabine.herbener@span.ch

History - Presentation - Activities. Created in 1987 by parents of twins and rapidly joined by adult twins, the association is open to anyone interested in twins and multiple births. It aims to bring quality information. The main core is the sharing of experiences by the volunteers.

The purposes of the regional representative:

\section{- Newsletters}

- Hotline for information and psychological help

- Organisation of meetings and participation in activities of similar associations

- Selling:

A) Files about specific themes like "parents-to-be of twin children or more" and "tricks for multiple birth".

b) Pins with logo.

- Visits to families and parents-to-be.

- Advertisement service for material help.

- Help to students and research workers.

It answers many questions from media without giving members personal information about members. Want to join us? Please check address and details above.
PSYCHIATRIC COMORBIDITY IN CHRONIC FATIGUE SYNDROME: A CO-TWIN CONTROL STUDY.

Richard Herrell, Niloo Afari, Susan Hartman, Megan Belcourt, Jack Goldberg, and Dedra Buchwald.

Correspondence address: Harborview Medical Center, 325 9th Avenue, Seattle, WA 98104

Objective. To examine the lifetime prevalence of psychiatric disorders in twin pairs discordant for chronic fatigue syndrome (CFS) and to determine symptom overlap of CFS and depression.

Methods. A sample of 68 twin pairs discordant for CFS completed a mailed questionnaire that collected information on symptoms of fatigue and depressive symptoms; subsequently the Diagnostic Interview Schedule-III-R was administered by telephone. CFS was defined according to the CDC research criteria. Levels of current depressive symptoms and lifetime psychiatric symptoms of depression were examined separately from a diagnosis of major depressive disorder.

Results. Large associations were found for CFS and PTSD (OR = 10.0, $\mathrm{CI}=1.3,78.1)$, major depressive disorder $(\mathrm{OR}=6.5, \mathrm{CI}=2.3,18.6)$, and panic disorder $(\mathrm{OR}=6.0, \mathrm{CI}=1.3,26.8)$. Analysis of current level of fatigue and individual symptoms of depression indicated increased levels of distress due to symptoms not shared with depression (such as dysphoria and anhedonia), in addition to large, anticipated associations with low energy and finding everything an effort.

Conclusion. Lifetime post-traumatic stress disorder, major depressive episode, and panic disorder are much more common in persons with CFS than in their unaffected co-twins. The presence of symptoms common to CFS and major depressive episode does not entirely account for the association between CFS and other lifetime and current depressive symptoms.

\section{$110 \mathrm{~F}$}

FETAL AND INFANT MORTALITY IN TWIN

\section{Lisa Hilder,Alastair G. Sutcliffe}

Correspondence address: Academic Department of Community Child Health, Royal Free

\& University College Medical School, Rowland Street, London, NW3 2QG

This paper reports estimates of gestation-specific fetal, neonatal and postneonatal mortality in twins compared with singletons, considering twins as pairs and as individuals. A population cohort of 171,527 birth notifications was obtained from prospectively maintained child health systems in North East Thames, 1989-1991. Deaths were validated by linkage with birth and death registration details. Multiple births (4234) were linked to form birth sibship for all but 124 (3\%) records, yielding 1989 pairs of twins.

Results. Trends in gestation-specific fetal mortality per 1000 women still pregnant $(/ 1000 \mathrm{sp})$ were compared between singleton and twin pregnancies. At all gestations risk of fetal mortality amongst twins is substantially higher and rises faster than that observed after singleton pregnancies. At 28-30 weeks risk of fetal loss was $2.1 / 1000$ sp in twins compared with $0.6 / 1000 \mathrm{sp}$ in singletons. At 37-39 weeks gestation the risk of fetal losses were $19.3 / 1000 \mathrm{sp}$ in twins and $1.6 / 1000 \mathrm{sp}$ in singletons. In contrast, neonatal deaths were concentrated at early gestations, and the risk of neonatal death decreased progressively in both singletons and twins with advancing gestation. Twin mortality was higher than singleton before 31 weeks. A window of advantage in survival was observed for twins (8/1000 live born) compared with singletons (12/1000 live born) between 31 and 36 weeks, most evident when considering each twin's survival individually. This advantage appears to persist in the postneonatal period. Weeks 32-35 represent a period when singleton birth is statistically not normal, and twin birth is.

Conclusions. Overall twins are at greater risk of mortality at all gestations. The improved survival for liveborn twins at 31-36 weeks does not compensate for increased fetal losses in this period.

\section{$111 \mathrm{~S}$ DIABETES AND SEMI-STARVATION DURING RAT PREGNANCY AND LACTATION: CONSEQUENCES FOR THE OFFSPRING.}

\section{K. Holemans}

Correspondence address: Dept of Obstetrics and Gynecology, Katholieke Univesiteit Leuven, Belgium

It is now evident that chronic diseases at adult age can be acquired during fetal life. Most epidemiological investigations focussed on the association between cardiovascular disease and low birth weight, but some studies also implicated maternal diabetes in adulthood disease of the offspring. To study the pathophysiology of the association between low birth weight and diseases in later life, animal models are used. We compared two rat models of perinatal growth retardation, diabetes mellitus (DR) and semistarvation (SR) of the maternal rat, in the effects on their offspring. 22-days old fetuses of DR and SR showed a comparable degree of growth retardation. Postnatally, body weight increased parallel to that of control rats, but there was no catch-up growth. Offspring of DR 
(O-DR) exhibit insulin resistance which involves both hepatic glucose production and peripheral glucose utilisation. Offspring of SR (O-SR), show insulin resistance in the liver but apparently not in the peripheral tissues. Determination of glucose metabolic index in individual tissues support these results. Mesenteric arteries from O-DR show a reduced endothelium-dependent relaxation and enhanced constriction to noradrenaline. Relaxation to acetylcholine and bradykinin is also reduced in arteries from O-SR, indicative of a reduction in $\mathrm{NO}$ synthesis. On the other hand relaxation to sodium nitroprusside is enhanced. This could reflect a compensatory response to tonic NO depletion. In summary then - in utero events may have lasting consequences in terms of insulin sensitivity and vascular function. Whether these may contribute to overt adult disease remains to be determined.

\section{F BIRTH WEIGHT, EARLY ENVIRONMENT, AND GENETICS - A STUDY OF TWINS DISCORDANT FOR ACUTE MYOCARDIAL INFARCTION}

Anna Hübinette, Sven Cnattingius,Anders Ekbom, Ulf de Faire, Michael Kramer, Paul Lichtenstein

Correspondence address: Department of Medical Epidemiology, Karolinska Institutet, P.O Box 281, SE-171 77 Stockholm, Sweden.Anna.Hubinette@mep.ki.se

Fetal growth is believed to be of importance in the aetiology of coronary heart disease (CHD). Epidemiological studies using birth weigh as a crude marker of fetal growth suggest that low birth weight is associated with increased risk of CHD. However, these studies had limited possibilities to control for potential confounding effects of genetic factors and of environmental influences other than fetal growth. In studies of twins, within-pair comparisons markedly reduce these potential confounding effects. This case-control study of acute myocardial infarction (AMI) was nested within the population-based Swedish Twin Registry, linked with the Cause of Death and Hospital Discharge Registries. We retrieved birth records containing information on birth and maternal characteristics from 132 like-sexed twin pairs discordant for AMI and 118 individually-matched control twin pairs, in which neither twin experienced AMI. In comparisons between AMI cases and external matched control twins, cases had significantly lower mean birth weight (143 g, $p=0.04)$, birth length $(0.8 \mathrm{~cm}, p=0.04)$, and head circumference $(0.5 \mathrm{~cm}, p=0.03)$ than controls. In within-pair comparisons between AMI cases and healthy co-twins, no significant differences in birth measurements were found. The lack of an association between birth weigh and AMI within twin pairs suggest that previously reported associations are confounded genetic and early environmental factors.

\section{$113 P$ DIGITAL POSTNATAL ANGIOGRAPHY} OF THE PLACENTA IN TTTS

Monique A Huisman, Ricardo N Laurini, Birgit Arabin

Correspondence address: Clara Angela Foundation Zwolle (NL) \& Witten (GER)

Aim of the study. After the pioneer work of Schatz in 1882 and of Benirschke in 1975 arteriovenous (AV) anastomoses are known to be a substrate of twin-to-twin transfusion syndrome (TTTS) in MC multiple pregnancy. We tried to evaluate whether placental digital angiography might add information about placental sharing, vessel distribution, type of AV shunting and the success or failure of Laser therapy.

Material and Methods. Eight cases of chronic TTTS without laser therapy, two cases with laser therapy and two cases with acute TTTS were up to now selected. After birth, the umbilical vessels of both twins were canulated, perfused with heparin and contrast medium under control of a computerangiograph.

Results. In chronic TTTS without laser treatment, we observed two types of AV shunts characterized by either thin trans-cotelydonary anastomoses or a thin kind of "end-to-end anastomoses" seaming to pass several cotyledons, of which the pregnancies were combined with a higher rate of perinatal mortality/morbidity. In one case with complete laser treatment hydrops of the recipient resolved and no (more) anastomoses were observed, in the other case ascites re-appeared after selective laser treatment and we detected small AV anastomoses by computerangiography though no anastomoses were verified by the conventional color injection method. In acute TTTS, the dye rapidly traversed through villous capillaries appearing on the venous side of the co-twin and the corresponding donor arteries and recipient veins penetrated the placenta extremely close so that the anastomoses soon appeared as a continuous vessel.

Conclusions: Digital computerangiography of the placenta helps to document the anatomical substrate of TTTS and the efficiency of laser coagulation and might improve our knowledge regarding etiology, therapeutic strategies and outcome.
Richard G ljzerman, Coen DA Stehouwer, Mirjam M van Weissenbruch, Eco J de Geus, Dorret I Boomsma

Correspondence address: Academic Hospital 'Vrije Universiteit', Department of Internal Medicine/Research, PO Box 7057, Amsterdam, The Netherlands

Several recent studies have demonstrated an association between low weight at birth and an atherogenic lipid profile in later life. To examine whether this association is explained by intrauterine or genetic factors, we investigated in 61 dizygotic and 53 monozygotic adolescent twin pairs. Birth weight was negatively associated with cholesterol, LDL cholesterol and apoB $(-0.17 \mathrm{mmol} / 1$ per $\mathrm{kg}\{95 \% \mathrm{CI}:-0.35$ to 0.01$\},-0.18$ $\mathrm{mmol} / 1$ per $\mathrm{kg}\{-0.36$ to -0.01$\}$ and $-0.07 \mathrm{~g} / 1$ per $\mathrm{kg}\{-0.11$ to -0.03$\}$, respectively), and positively with HDL cholesterol $(0.04 \mathrm{mmol} / \mathrm{l}$ per $\mathrm{kg}$ $\{-0.02$ to 0.10$\})$, after adjustment for age, sex and BMI. Intrapair differences in birth weight were significantly and negatively associated with differences in cholesterol, LDL cholesterol and apoB in dizygotic twins $(-0.49 \mathrm{mmol} / 1$ per $\mathrm{kg}\{-0.89$ to -0.08$\},-0.51 \mathrm{mmol} / 1$ per $\mathrm{kg}\{-0.90$ to -0.13$\}$ and $-0.10 \mathrm{~g} / \mathrm{l}$ per $\mathrm{kg}\{-0.19$ to -0.01$\}$, respectively), but positively in monozygotic twins $(+0.32 \mathrm{mmol} / \mathrm{l}$ per $\mathrm{kg}\{0.03$ to 0.62$\}, 0.23$ $\mathrm{mmol} / \mathrm{l}$ per $\mathrm{kg}\{0.03$ to 0.49$\}$ and $0.06 \mathrm{~g} / \mathrm{l}$ per $\mathrm{kg}\{-0.03$ to 0.12$\}$, respectively), after adjustment for BMI. The positive association between intrapair differences in birth weight and differences in HDL cholesterol was not significant in dizygotic twins $(0.04 \mathrm{mmol} / \mathrm{l}$ per $\mathrm{kg}\{-0.10$ to 0.16$\})$ and of borderline significance in monozygotic twins $(0.11$ $\mathrm{mmol} / \mathrm{l}$ per $\mathrm{kg}\{0.00$ to 0.23$\})$. These data suggest that genetic factors account for the negative association of birth weight with cholesterol, LDL cholesterol and apoB, whereas intrauterine factors possibly play a role in the association between birth weight and HDL cholesterol.

\section{$115 S$ TRENDS IN TWINNING, TRIPLET, AND OTHER MULTIPLE BIRTHS IN DEVELOPED COUNTRIES, 1972-1999}

\section{Yoko Imaizumi}

Correspondence address: Faculty of Health Science, Hyogo University, Kakogawa City, 675-0101, Japan

Yearly changes in twinning, triplet, and quadruplet rates were analyzed using vital statistics in Austria, the Czech Republic, the Slovak Republic, England and Wales, Germany, the Netherlands, Switzerland, Finland, Norway, Sweden, Hong Kong, and Japan, during the period 1972-1999. Among those 12 countries, the twinning rate in the Slovak Republic remained constant from 1972 to 1999 , whereas the twinning and triplet rates in the Czech Republic, and the triplet rate in the Slovak Republic remained constant from 1972 to 1994 , and increased thereafter. On the other hand, the twinning rate increased significantly year by year in the other 10 countries during the examined period. Twinning rates increased from 1.4-fold in Finland and Austria to 2-fold in Norway from 1972 to 1998. As for triplets, triplet rates increased up to 1991-1993 and decreased thereafter in the Netherlands, Finland, Norway, and Sweden. In Austria and Germany, triplet rates increased significantly year by year during that period. In the other four countries, the rate increased up to 1994-1997 and decreased or remained thereafter. Triplet rates increased from 2.3-fold in Finland to 12.4-fold in Austria during that period. As for quadruplets, the rate increased up to 1990 in Germany, 1994 in England and Wales, 1995 in Japan and decreased hereafter. The rising multiple rates have been attributed to the higher proportion of mothers treated with ovulation-inducing hormones and partially attributed to IVF.

\section{F GENETIC AND ENVIRONMENTAL INFLUENCES ON THE RELATIONSHIPS BETWEEN ANTISOCIAL BEHAVIOR AND ALCOHOL ABUSE AND DEPENDENCE IN MALE TWINS}

Kristen C Jacobson, Carol A Prescott, Michael C Neale, Kenneth S Kendler Correspondence address: VIPBG; MCV/VCU; PO Box 980126; Richmond,

Va 23298-0126, USA

Previous research has revealed that both child conduct disorder (CD) and adult antisocial behavior (AAB) predict adult alcohol abuse and dependence (ALC). However, research concerning genetic and environmental influences on the relationship between antisocial behavior and ALC has yielded inconsistent results. This study used reports of CD symptoms prior to age 18 , AAB symptoms after age 18 , and lifetime ALC symptoms from a population-based sample of 3520 adult male twins to estimate the genetic and environmental influences underlying the comorbidity among the 3 behaviors, and in particular, to test whether the relationships between the 3 behaviors could be explained by a single underlying factor. A common pathway model fit the data equally as well as a model allowing for independent genetic and environmental factors, suggesting that covariation among the 3 behaviors is determined by a single underlying latent factor, and that the relationships between $\mathrm{CD}$ and ALC and between AAB and ALC have similar genetic and envi- 
ronmental etiologies. Genetic, shared environmental, and nonshared environmental factors explained $35 \%, 27 \%$, and $38 \%$ of the variance in this latent factor, respectively. There were additional sources of familial resemblance for $C D$ and ALC that were not shared with the underlying latent variable: phenotype-specific genetic factors explained an additional $32.5 \%$ of the variance in ALC, and phenotype-specif ic shared environmental factors accounted for an additional $18.5 \%$ of the variance in $C D$.

\section{$117 F$ THE COVARIANCE STRUCTURE OF NEUROTICISM AND AGREEABLENESS: A TWIN AND MOLECULAR GENETIC ANALYSIS OF THE ROLE OF THE SEROTONIN TRANSPORTER GENE}

Kerry L. Jang, Stella Hu,W. John Livesley,Alois Angleitner, Rainer Riemann, Juko Ando, Yutaka Ono, Philip A.Vernon, \& Dean H. Hamer

Correspondence address: Division of Behavioural Science, Department of Psychiatry University of British Columbia, Vancouver, British Columbia, Canada

Personality trait domains, such as neuroticism and agreeableness are considered factorially distinct, despite a number of correlations between the domains. The genetic and environmental correlations that index the degree to which the observed relationship between the Revised NEO Personality Inventory scales of Neuroticism and Agreeableness are caused by genetic or experiential influences were estimated using data from 913 monozygotic (MZ) and 562 dizygotic (DZ) volunteer twin pairs from Canada, Germany, and Japan. The serotonin transporter gene, 5-HTTLPR, was assayed in a sample of 338 non-twin sibling pairs from the United States to determine the contribution of the serotonin transporter locus to the covariation between the Neuroticism and Agreeableness scales. In all four samples genetic influences, as opposed to environmental influences, contributed to the covariance of Neuroticism and Agreeableness, with the serotonin transporter gene accounting for $10 \%$ of the relationship between these domains. The implications of these results on models of personality structure, taxonomy, and measurement are discussed.

\section{$118 \mathrm{P}$ GENETIC INFLUENCE ON MARITAL STATUS Beth A. Jerskey, Michael J. Lyons, Courtney E. Lynch, Denise A. Hines, Simone Ascher and Ming Tsuang \\ Correspondence address: Boston University, 64 Cummington Street, Boston, MA 02215}

Previous research demonstrating differences in $\mathrm{MZ}$ versus DZ similarity for divorce suggests that genetic factors may play a significant role. However, there has not been a great deal of work that includes "neve married," "currently married," "currently divorced," and "divorced and remarried" in the same series of analyses. To address this issue, we used data collected from members of the Vietnam Era Veteran Twin Registry. The Registry comprises male twin pairs who served in the U.S. military between 1965 and 1975. In 1987, approximately 8,000 twins from the original sample were interviewed by telephone using the Diagnostic Interview Schedule to diagnose mental disorders. Our preliminary analyses indicate that there is a genetic influence on whether an individual has ever been married and there is also a genetic influence on whether an individual who has been married has ever been divorced. We will present results of analyses that assess whether the genetic and environmental factors that influence whether an individual marries are the same as the genetic and environmental factors that influence whether he eve divorces. We will also assess the role that psychopathology may play in mediating the relationship between genetic factors and marital history.

\section{F THE COST-EFFECTIVENESS OF IN VITRO FERTILISATION}

\section{Christopher Jones, Ryk Ward}

Correspondence address: Institute of Biological Anthropology, University of Oxford, 58 Banbury Road, Oxford OX2 6QS (UK)

The issue of how many embryos to replace per cycle of in vitro fertilisation has been at the forefront of recent clinical debate. We investigated this issue in the context of cost-effectiveness. Cost-optimisation model were applied to one of the World's largest IVF datasets $(93,295$ patients who underwent 174,418 IVF treatment cycles in the United Kingdom from 1991-1998). We calculated the most cost-effective number of embryos per cycle in light of two competing variables - cycle costs and costs of live deliveries. Total savings were calculated by evaluating the consequences of transferring patients from an expensive group to a less-expensive one. Our analyses indicate the current practice of transferring more than two embryos results in an unacceptable ethical and financial burden, due to the increased risk of multiplets. Minimising delivery costs with respect to cycle costs would yield immediate benefits. FOR SUPERTWINS

Noriko Kato, Masako Kobayashi

Correspondence address: National Institute of Public Health 4-6-1 Shirokanedai, Minato-ku, Tokyo 108-8638Japan kato@iph.go.jp

In order to make birthweight reference curves for triplets and quadruplets, magnetic tape records of birth and stillbirth certificates from 1988 to 1991 were analyzed. These included 1894 triplet live births from 744 sets of triplets. 206 quadruplet live births were analyzed. The 10th, 50th and 90th percentile values were calculated for each gestational week. Median birthweight for gestational age was found to be highest in twins, lower in triplets and lowest in quadruplets. In triplets, the 50th centile for boys was $0.08 \mathrm{~kg}$ heavier than girls. Quadruplet girls were smaller than boys under 29 weeks and larger above 30 weeks. Growth standards for the exclusive use in triplets and quadruplets seem to be necessary.

\section{P PERINATAL MORTALITY OF TWINS IN RECENT JAPAN}

Noriko Kato, Masako Kobayash

Correspondence address: National Institute of Public Health, 4-6-1 Shirokanedai,

Minato-ku,Tokyo 108-8638 Japan kato@iph.go.jp

This study is intended to clarify the difference in secular trends of perinatal mortality between like-sexed and unlike-sexed twins. From twins born from 1975 to $1994,368,045$ birth certificates, 42,172 still-birth certificates, and 7,711 early neonatal death certificates were analyzed. 97.8\% of twin pairs and $96.5 \%$ of corresponding birth and death certificates pairs were identified. Perinatal mortality rates were calculated every year from 1979 to 1994. Logistic regression analysis was undergone with dependent variables of perinatal deaths in the years 1984 \& 1994 . Peninatal mortality rate decreased with the same speed in like-sexed and unlike-sexed pairs from 1979 to 1984, while after 1985, decrease became slow in unlike-sexed pairs. Odds ratio of unlike-sexed was $0.56(0.41-0.78)$ in 1984 compared to $0.72(0.54-0.97)$ in 1994 , with the baselines of like-sexed twins.

\section{F TWIN FESTIVALS TO FACILITATE TWIN RESEARCH}

\section{D.M. Keith, L.G. Keith}

Correspondence address: Center for Study of Multiple Birth, 333 E. Superior St., Chicago IL 60611

The main obstacle to cost effective twin research is a lack of a sizable population to obtain an appropriate sample. Twin Festivals offer a means to overcome these barriers. Two festivals are ideally suited for twin research: the Twinsburg Ohio Twinsdays Festival and the All European Twins Festival, held in Szczecin, Poland. The authors have conducted field research at the Twinsdays Festival in the United States for over 20 years. The main value of this work is that "you can do three years of research in three days." Certain limitations apply. First, an IRB approval is required; second, a "space" for the work. Then, the Team Lead must assemble and train his team to carry out the project in an environment that lacks the normal day to day support available in most professional institutions. All aspects of the research must be laid out and divided into stations to accommodate LARGE number of research participants. Everything that could be required for the project must be assembled, packed, and loaded for the trip to the site. Festival participants are plentiful and eager to participate in the research. Parents are most cooperative. They realize their children can make a difference and allow them to take part because they are a very special population group. Plans are necessary for departing the site. Since the festivals are two days long, a decision must be made on the length of the project. The most gratifying part of the field research is in the initial review of the date when the team realizes they have obtained information they need.

\section{$123 \mathrm{P}$}

\section{THE ROLE OF NHS MULTIPLE BIRTHS CLINICS IN THE UNITED KINGDOM}

\section{Anne M Kelly Christine Burton.}

Correspondence address: Children's Centre, York District Hospital, Wigginton Road, York.

Dr Elizabeth Bryan founded the Multiple Births Foundation in 1988 with the twin aims of informing professionals and providing some direct services to families. The first Multiple Births clinic was held at Queen Charlotte's and Chelsea Hospital, London in 1987. Satellite MBF clinics developed soon afterwards in York and Birmingham operating on an open referral system. The clinics were held in community premises in Birmingham and in a child development centre in York. Local paediatric and health visitor staff with some volunteers staffed them, together with the visiting MBF paediatrician. MBF withdrew from active participation in these clinics in 1997-8. The Birmingham clinic came under the auspices of the Special Community Health Trust. The clinic in York became part of the paediatric outpatient service. These are the only National Health Service multiple births clinics in the country. They offer 
full paediatric assessment as well as support and advice to the families of twins and multiples. Senior paediatricians and health visitors who staff the clinics are able to offer specialist advice including bereavement support. The staff liase with other professionals locally to promote knowledge and awareness of the needs of the families of multiple births. Clinics are held monthly in Birmingham and accept open referrals, whilst in York clinics are held bi-monthly and referral by a general practitioner is required. The most common concerns relate to growth, development, emotional and behavioural problems, and general health in addition to queries about zygosity determination. The highest rate of referral is for young children aged $<5$ years, in common with referral patterns generally across paediatrics.

\section{F GENETIC AND ENVIRONMENTAL INFLUENCES ON ACNE IN ADOLESCENT TWINS}

Katherine M. Kirk, David M. Evans, Brendan Farthing and Nicholas G. Martin Correspondence address: Queensland Institute of Medical Research, Post Office, Royal Brisbane Hospital, QLD 4029 Australia

Acne is an extremely prevalent skin condition primarily affecting adolescents, but often continuing into adulthood. It consists of inflamed or non-inflamed lesions on the face and trunk, and in its severe form has been shown to be associated with psychological factors such as anxiety and poor self-esteem. Measures of acne severity on the face, chest and back were obtained from male and female adolescent Australian twins: $105 \mathrm{MZ}$ and $279 \mathrm{DZ}$ pairs were measured at age 12 , and $139 \mathrm{MZ}$ pairs and $274 \mathrm{DZ}$ pairs at age 14. Facial acne data was also collected from 126 $\mathrm{MZ}$ and $185 \mathrm{DZ}$ pairs at age 16. Significant genetic influences on facial acne were detected in males and females at all three ages considered, with univariate heritability estimates between 0.5 and 0.9 for girls, and between 0.7 and 0.8 for boys. No significant shared environmental influences were found for facial acne. Genetic influences were also found to be important for acne on the chest and back at age 14, and preliminary multivariate analyses indicate that there may be both common and specific genetic influences on acne at the three sites considered.

\section{P GENES AND ENVIRONMENT IN FEBRILE} SEIZURES: A POPULATION-BASED TWIN STUDY

\section{Marianne J. Kjeldsen, Kirsten O. Kyvik, Kaare Christensen, Mogens L. Friis.} Correspondence address: The Danish Twin Registry, Institute of Public Health, Epidemiology, University of Southern Denmark, Odense.

Purpose. To examine the role of genetic and environmental factors on the aetiology of febrile seizures (FS) and to estimate the degree of heritability. Methods. Data on self-reported FS was collected on 34,076 twin individuals, aged 12-41 years and originating from the Danish Twin Registry. The data was analysed using the classic twin study design. Probandwise concordance rates, odds ratios and tetrachoric correlations were calculated and used to assess the degree of similarity in monozygotic (MZ) and dizygotic (DZ) twins. By model fitting different combinations of genetic effect (additive or dominant) and effects of environment (shared or non-shared) were evaluated and the heritability of FS was estimated.

Results. 29,179 twins responded to the questionnaire. Excluding twins with unknown zygosity, health history information on FS was obtained from both twins in 11,872 pairs. A total of 805 cases of FS were found. Probandwise concordance rates, odds ratios and tetrachoric correlations were significantly higher for $\mathrm{MZ}$ than for $\mathrm{DZ}$ twins. According to biometrical modelling, a model combining additive genetic effects and environmental effects that were specific to the individual provided the best overall fit to the data. From this model a heritability of FS of 0.69 was found. No sex difference or age-dependency in the magnitude of genetic effects was observed.

Conclusions. These results show a major genetic component in susceptibility to FS although a substantially part of the aetiology of FS is due to environmental effects that are not shared by the twins in a pair.

\section{$126 \mathrm{P}$}

\section{GENETIC EPIDEMIOLOGY OF ALCOHOL DEPENDENCE IN A HEAVY DRINKING NATIONAL TWIN COHORT}

Valerie S. Knopik, Andrew C. Heath, Pamela A. F. Madden, Kathleen K. Bucholz, Elliot C. Nelson, and Nicholas G. Martin

Correspondence address: Department of Psychiatry, Washington University School of Medicine, 40 N. Kingshighway, Suite 1, St. Louis, MO 63108

In order to explore risk factors for DSM-IV alcohol dependence in both men and women, diagnostic telephone interview data from young adult Australian twin pairs born 1964-1971 were analyzed using Cox models and structural equation modeling techniques. Cox regression models were fitted to interview data from a total of 2709 complete twin pairs and from 766 single members of twin pairs. Results of these analyses suggest that the risk for alcohol dependence is increased in males, in Roman
Catholics, in those who report a history of major depression, panic disorder, and conduct disorder, or (in females only) a history of suicide attempt; whereas there is a decreased risk in those reporting Baptist, Methodist, or Orthodox religion, in males who report weekly church attendance, and in university-educated males. Controlling for these sociodemographic and psychiatric predictors, alcoholism rates remain significantly higher in the co-twins of alcoholic MZ compared to DZ alcoholic twins. Further, structural equation models fitted to observed summary statistics for each zygosity group (i.e., the numbers of concordant alcoholic, discordant, and concordant unaffected twin pairs, and of alcoholic and unaffected singleton twins) suggest that $53 \%$ of the total variance in alcoholism liability is attributable to additive genetic effects, $0 \%$ to shared environmental factors, and $47 \%$ to non-shared environmental influences. (Supported by NIH grants AA11998, AA10249, AA0758O, DA12854).

\section{P A TWIN STUDY OF GENETIC AND ENVIRONMEN- TAL FACTORS IN THE RELATIONSHIP BETWEEN ASTHMA AND BEHAVIOUR IN FOUR-YEAR-OLDS}

Gesina Koeppen-Schomerus,J Stevenson, R Plomin

Correspondence address: Institute of Psychiatry, King's College London, SGDPRC, 113 Denmark Hill, London SE5 8AF, UK.

Although the genetic and environmental factors of asthma have been extensively researched, no studies on the early development of asthma in children have been reported to date. The objective of the present study was to test in a large and representative sample of young twins the hypotheses that genetic influences are substantial and shared environmental influences are modest. Furthermore, the relationship between asthma status and behaviour problems and anxiety was also analysed. The sample consisted of 4910 four-year-old twin pairs who were born in England and Wales in 1994 and 1995 who were enrolled in the Twins Early Development Study (TEDS). Data on asthma status and behavioural assessments were obtained from the twins' parents by postal questionnaire.

Univariate analyses using model-fitting derived estimates of $68 \%$ for heritability, $13 \%$ for shared environment, and $19 \%$ for nonshared environment. Multivariate analyses found a relationship between asthma status and anxiety. Asthmatic children had significantly higher mean scores than non-asthmatic children for measures of anxiety $(p<.001)$, but there were no significant differences in other areas of development such as cognition or language.

Our findings show that asthma is highly heritable in 4-year-olds whereas shared environmental influences are not statistically significant. Furthermore, we found higher levels of anxiety in children with asthma. Multivariate genetic findings on asthma and anxiety will be presented.

\section{$128 \mathrm{~S}$}

THE CONTEXT AND LONG-TERM IMPACTS OF MULTIPLE BIRTH LOSS

\section{Jean Kollantai}

Correspondence address: Center for Loss in Multiple Birth, Inc., Anchorage, Alaska

As a result of our own experience with the death of a twin baby, we have worked on a fulltime basis for 13 years with more than 6,000 bereaved parents of twins, triplets or other high multiples throughout the US, Canada and other countries, of many socioeconomic, ethnic and racial backgrounds, ages and worldviews, with many situations: the death during pregnancy, at birth or after of both or all babies, of one twin, of one or more supertwins; selective reduction or termination, or the consideration of them, with and without later loss; pregnancy with a multiple who will or may die; the death of one or more multiples in childhood; and those with more than one multiple pregnancy and loss. Many original or early members continue as members or in touch, while new contacts arrive often at the rate of 5-7 daily, along with many others from professionals and multiples organizations. Of those who have survivors, many are the parents of one or more medically fragile or handicapped babies, some extremely so; and with technology, some surviving multiples are living for a time but dying in childhood. The presentation will summarize what can reasonably be inferred about the short- and especially the long-term impacts on parents and issues for any surviving or other children, in the societal context of the boom in multiple births and in media attention, as well as ART generally and other "multi-realities" and their implications for appropriate support to families as well as maximum efforts to prevent loss and thereby long-term complicated grief and its potential consequences. Materials will be available. 

IN ASTHMA AND HAY FEVER

\section{Markku Koskenvuo.}

Correspondence address: Department of Public Health, University of Turku, Lemminkäisenkatu 1, FIN-20520 Turku.

Background. Inherited susceptibility have been shown to play an important role in the development of asthma and hay fever. However, no definitive major liability gene has been detected, but some infections have been found to operate in the etiology.

Samples and methods. Three samples are presented: The "old" Finnish Twin Cohort represents the adult same-sexed twin pairs in 1975 with 13888 twin pairs with both co-twins responding to the questionnaire. Their hospital records subsequent to 1975 have been identified by record linkage. The "FinnTwin16" consists of questionnaire data of 2550 twin families with twin pairs aged 16 and born in 1975 to 1979 . "Allergy95" consists of questionnaire data of 10667 first-year university students in 1995 . The validity of diagnoses were clinically assessed in a sub-sample. Findings. The heritability of asthma among adult twins was $36 \%$, and among adolescent twins $75 \%$. Hay fever showed similar trends Among young adults the number of older siblings correlated negatively to the occurrence of hay fever and asthma. Among students the childhood farm environment reduced significantly the risk of hay fever (odds ratio 0.63 ), and asthma (OR 0.71) in models adjusted for day care and passive smoking at age of 0-2 years, gender, parents' asthma and atopy, and number of older siblings.

Conclusions. The heritability of asthma and hay fever has possibly increased during the last decades. In that case, environmental factors have increased the expression of liability genes. A good candidate for it is the delay or lack of some infections. Some early childhood gastrointestinal infections seem to protect from allergen sensitization. More evidence is needed from the role of infections in the genetic liability to allergic diseases.

\section{S CURRENT DIRECTIONS IN PERSONALITY RESEARCH WITH TWINS}

Robert F. Krueger

Correspondence address: Department of Psychology, University of Minnesota, Minneapolis, MN 55455, USA

Twins have contributed fundamentally to our understanding of the origins of individual differences in personality. Research with twins challenges classical notions of the family-environmental origins of personality. As a direct result, twin research has fundamentally reshaped the field of personality psychology by focusing personality psychologists on genetic and extra-familial origins of personality. Yet, in the age of molecular genetics, do twins still have things to teach us about personality? This presentation focuses on multivariate studies of personality and its correlates in twins and suggests that twins will continu to be fundamental to the field of personality psychology. Multivariate twin studies emphasise the fundamental role of personality in adjustment and maladjustment by suggesting that much of the genetic variance in key personality correlates is shared with the genetic variance in personality per se. Such research underlines personality as the key construct in understanding human adjustment and maladjustment, a per-spective that could not be achieved without the involvement of twins in personality research.

\section{P}

\section{DELIVERY OF PREVIABLE TWINS: SUCCESS RATES IN ATTEMPTED DELAY OF DELIVERY OF THE SECOND TWIN}

\section{Richard B. Kurze \\ Correspondence address: Dept. of OB/GYN, Mount Sinai Hospital and Chicago Medical School, Chicago, IL USA}

Objective. To determine the success rate when attempting delay of delivery of a second previable twin.

Study design. A 7-year experience of 158,872 deliveries included 1794 sets of twins. With delivery at a previable gestation (18-26 wks), delivery of the 2nd twin was delayed using tocolysis, antibiotics, with or without cerclage. The "success" rate - achieving survival of twin-B was determined, as well as the percentage excluded from intervention on obstetrical grounds.

Results. $4.7 \%$ of twin pregnancies $(n=84)$ delivered between $18-26$ wks gestation. Of these, only $26.2 \%$ were candidates for delay of delivery of twin-B on OB grounds. Intervention was attempted on only 8 patients, with a $37.5 \%$ success rate.

Conclusion. All successes were in patients where cerclage was used in conjunction with tocolysis and antibiotics. Aggressive intervention could potentially save $10 \%$ of previable 2 nd twins.
132P HLA HAPLOTYPES IN DIZYGOTIC TWIN PAIRS

Kirsten Ohm Kyvik, Ingrid Louise Titlestad, Tom Kristensen, Søren Lillevang. Correspondence address: The Danish Twin Registry, Epidemiology, University of Southern Denmark, Odense, Denmark

It has been suggested that dizygotic twin pairs share two HLA haplotypes more often than ordinary siblings and thus might be more genetically alike. We tested this hypothesis in dizygotic twin pairs from the Danish Twin Registry.

Subjects and methods: A total of 114 (60 female and 54 male) samesexed healthy twin pairs aged 18-45 years participated. Dizygosity was confirmed by means of DNA sequencing of nine polymorphic markers. HLA-A, B and Cw specificites were typed with serology, and if data were inconclusive, with DNA typing. If twin partners had the same HLA-types, they were assumed to share two haplotype. If they had 1 HLA type in common they were assumed to share one haplotype and if they had no HLA types in common they were assumed to share zero haplotypes. Since HLA-types from parents were unavailable we could not test for identity-by-descent and thus have a risk of overestimating the number of twins sharing two haplotypes. A Chi-square test was used to compare observed numbers in each haplotype-sharing group with the expected numbers.

Results. Twentynine (expected 28.5) twin pairs had two, 52 (expected 57) had one and 33 (expected 28.5) had zero HLA-types in common, $p=0.56$

Conclusion. Dizygotic twin pairs are not more similar than ordinary siblings born from different pregnancies.

\section{$133 S$ THE ENDOCRINOLOGY OF DIZYGOTIC TWINNING IN THE HUMAN}

\section{Cornelis B. Lambalk}

Correspondence address: Division of Reproductive Medicine of the department

of Obstetrics and Gynaecology and the Research Institute of Endocrinology, Reproduction

and Metabolism, Vrije Universiteit, Medical Centre, Amsterdam.

Heredity, higher maternal age and increased parity are well-defined conditions associated with dizygotic twinning. An endocrine model of excessive secretion of pituitary gonadotrophic hormones explains multiple ovulation as a result of multiple follicle growth. In hereditary conditions FSH levels are indeed clearly elevated because of increase in stimulating mechanisms that regulate pituitary gonadotropin secretion while in most non-hereditary conditions, overshoot FSH secretions occurs as a result of diminished ovarian feedback. Puberty is a condition in which the hypothalamic LHRH pulse generator is reinitiated and this is typically characterized by temporary overshoot LH and FSH secretion, probably due to not yet fully operational ovarian feedback. In adult females situations can be found that mimic this peripubertal event such as while recovering from hypothalamic amenorrhea. Under these circumstances more DZ twinning can be observed. Elevated FSH levels along with ageing in premenopausal women probably underlie the age related increased risk of dizygotic twinning. The apparent paradox in the combination of age related decline in fecundity and rise in twinning risk can be explained by incidental presence in the cohort of more than one follicle, containing vital oocytes under deficient feedback mechanisms that lead to high FSH.

\section{F MULTIPLE PREGNANCY AND PERINATAL DEATHS AFTER ASSISTED CONCEPTION}

\section{Paul A.L. Lancaster and Tara L. Hurst}

Correspondence address: AIHW National Perinatal Statistics Unit, The University of New South Wales and Sydney Children's Hospital, Randwick, NSW 2052, Australia

The twinning rate in Australia increased by more than $60 \%$ from 9.0 per 1,000 confinements in 1977 to a peak of 14.5 per 1,000 in 1998 . Among 251,650 births in 1998, assisted conception (IVF, ICSI, and GIFT, but not fertility drugs alone) accounted for $15.3 \%$ of all twin births and $47.2 \%$ of triplets and higher order multiples.

We analysed data from the national register of assisted conception for Australia and New Zealand and compared outcomes with those for all births in Australia. Among 33,162 infants conceived between 1979 and 1998, there were 689 fetal deaths (20.8 per 1,000 total births), 440 neonatal deaths (13.5 per 1,000 live births), and 1,129 perinatal deaths (34.0 per 1,000 total births). Perinatal death rates were lower in the most recent years but the decline was more pronounced for singleton than for multiple births. In 1993-98, the perinatal death rate of singleton infants born after assisted conception $(18.4$ per 1,000$)$ was twice that for all singleton births in Australia $(9.1$ per 1,000) but the rates for twins (43.0 and 42.4 per 1,000) and higher order multiples (103.6 and 94.6 per $1,000)$ were similar. Multiple births accounted for $59.8 \%$ of perinatal deaths after assisted conception compared with $12.8 \%$ in the population. Until fewer embryos are transferred in IVF procedures, multiple births will continue to be the major adverse outcome of these pregnancies. 


\section{$135 S$ EXAMINING THE LEGALITY OF MANDATORY TWIN SEPARATION}

\section{Lana Larson Dean}

Correspondence address: 2953 Autumn Run Place, Orlando, Florida, USA 32822

In her presentation, Lana Larson Dean will explore the disparity among American parents' constitutional rights involving the education and upbringing of their children. By reviewing past and current case law, Ms. Dean will address the unique constitutional questions that arise when parents are faced with mandatory separation of their twins in the classroom. Finally, Ms. Dean will comment on the likelihood of prevailing in a lawsuit in these circumstances and will suggest alternative means of resolving the separation issue.

\section{$136 \mathrm{~S}$ LOOKING BEYOND THE TECHNOLOGY: COPING WITH HIGHER ORDER MULTIPLES}

\section{Donna Launslager}

Correspondence address: Multiple Births Canada, 248 Tatlock Court, Waterloo,

Ontario N2L $5 Y 6$

In recent years, much of the focus on Assisted Reproductive Technologies (ART's) has been directed to issues surrounding the rights of people using reproductive services, genetic manipulation and surrogate motherhood. Little attention has been given to the impact of ART's on the family and the children — the desired outcome of these services. There is an urgent need to examine all ART's issues and to look past the technology to its overall effect on the children and the families the children are born to. As a mother of quadruplets, discussion will focus on what it means for a parent to suddenly have several newborns added to the family — the physical, psychological and financial pressures.

\section{$137 S$ PLACENTAL PATHOLOGY IN DISCORDANT TWINS}

\section{Ricardo N Laurini}

Correspondence address: Department of OB/GYN, University Hospital, 4200 Porto, Portugal As multiple gestations are a priori high-risk pregnancies, all such placentas must be examined and sent fresh immediately after delivery. This is essential for a number of examinations (e.g. microbiology, cytogenetics) including injection of chorionic vessels. All umbilical cords must be clearly identified as to twin of origin. If a radiological examination of the vascularisation is planned, the umbilical cords must be cannulated immediately and perfused following a standard protocol. In our experience such a comprehensive morphological examination clearly benefits from postnatal computerangiogram in evaluating the vascular structures including superficial and deep anastomoses and placental sharing (a poorly understood cause of discordancy). The morphological examination should establish the pattern of placentation and zygosity, pathology related to monochorionic placentation, as well as the types of pathology also seen in singleton placentas. The histological examination must include samples from the decidua basalis to assess the spiral arteries.

Different forms of placental pathology can be the cause of discordant twins and these can also act as confounding variables with respect to a possible TTTS. In addition to conventional vascular pathology, these causes of asymmetrical expression can include cases with a partial mole or a diffuse relative immaturity of one half of the placenta associated with a marked IUGR that resembled a TTTS situation with discordant twins. As for singleton placentas, it is important to explore the correlation between blood flow results and placenta morphology in dichorionic twin pregnancies with discordant growth. Therefore, we will discuss the cases presented by Peter Malcus et al.

\section{S THE BIOLOGY OF MONOZYGOTIC TWINNING: GENERAL ASPECTS}

\section{Fernand Leroy}

Correspondence address: 18, Marnix gv, 3090 Overijse, Belgium

The etiology of MZ twinning still eludes us. Also, in spite of some insights on the developmental mechanisms leading to the three main types of MZ twins (i.e. dichorial, DC : monochorial, MC and monoamniotic), a number of fundamental problems remains unsolved:

1. Gametogenic and/or developmental events leading to the rare occurrence of heterokaryotic twins.

2. Particulars of the mechanism of total or partial double gastrulation producing monoamniotic or conjoined twins, respectively.

3. Specificities of the formation and the implantation of MZ-MC twin concepti and their impact on placental vascularisation.

4. The mechanism(s) of MZ-DC twinning. It is currently debated whether such twins arise from separation of early blastomeres or from later division of the hatching blastocyst. The respective merits of these conflicting hypotheses will be examined.
Finally, two fundamental questions arise about the etiology of MZ twinning:

- Do they arise from a unique primary cause or might their causation be of dual nature?

- Bilateral symmetry is a very general feature of animal anatomy and $\mathrm{MZ}$ twinning can be looked upon as resulting from an exaggeration of the underlying developmental mechanisms. Thus, how is body symmetry genetically programmed?

\section{P SUBJECTIVE REACTIONS TO ACUTE NICOTINE: EFFECTS OF GENDER AND FAMILY HISTORY OF NICOTINE AND ALCOHOL CONSUMPTION}

Christina N. Lessov, Pamela A. F. Madden, Erik J. Sirevaag, John W. Rohrbaugh,

Ovide F. Pomerleau, and Andrew C. Heath

Correspondence address: Washington University School of Medicine, St. Louis, MO

The degree of initial sensitivity to the effects of nicotine may represent a biological predictor of nicotine dependence vulnerability. In a sample of nonsmoking (smoked less than 100 cigarettes lifetime) young adult twins and singletons (aged 18-23 years, total $n=75$ ), subjective ratings were obtained $10 \mathrm{~min}$ following placebo or nicotine (Nicotrol nasal spray, $1.0 \mathrm{mg}$ ) administration. On an item-by-item basis, following nicotine, women reported a greater degree of dizziness $(p<0.05)$ and men reported a greater degree of calmness $(p<0.001)$. A factor analysis was conducted separately for each sex, and a 3-factor solution was found to adequately describe the data; we called these factors 'stimulation', 'sedation' and 'physical sensation'. Similar factor patterns were defined for women and men. Parental history of nicotine dependence was significantly associated with the 'stimulation' factor for both women and men (both $p<0.05$ ); parental history of excessive drinking was significantly associated with the 'stimulation' factor only in men $(p<0.05)$, and was significantly associated with the 'physical sensation' factor only in women $(p<0.05)$. The 'sedation' factor, and self-reported feelings of dizziness or calmness were not significantly associated with family history of smoking or drinking. The results suggest that gender differences exist in subjective reactions to acute nicotine, which may be due to gender differences in neural sensitivity to some effects of nicotine, in addition to gender-specific association with family history of nicotine and alcohol use. (Supported by grants DA07261, DA00272, and CA75581)

\section{$140 \mathrm{~S}$}

\section{CEREBRAL PALSY IN TWINS:} DOES IT HAVE A DIFFERENT AETIOLOGY?

Malcolm I Levene

Correspondence address: Academic Unit of Paediatrics, University of Leeds School of Medicine, Clarendon Wing, Leeds LS2 9NS, UK

Cerebral palsy is the commonest motor disability in childhood and is more likely to occur in multiple births than in singletons. The risk of cerebral palsy in twins is six-fold increased and in triplets is 20 fold increased compared to singletons. The reasons for this are multifactorial, but there are two important factors that appear to predispose twins and higher multiples to cerebral palsy. The first is the increased risk of preterm birth. This risk increases significantly with the number of fetuses in utero. The median gestational age of delivery of triplets is 32-33 weeks and for quadruplets is 30-31 weeks. Severe prematurity exposes the babies to risks of intracranial pathology secondary to lung disease. The commonest cause of cerebral palsy is periventricular leukomalacia in this group, which is most commonly associated with the subsequent development of spastic diplegia. It is also well recognized that mature twin delivery is also associated with a significant increased risk of cerebral palsy. This is particularly common in monozygotic twins where twin-to-twin transfusion syndrome (TTTS) occurs in 5-15\% of such pregnancies. This risk may be reduced by obstetric intervention early in pregnancy but the risk of neurodevelopmental disability in TTTS is in the order of $25 \%$. This is particularly likely if there has been the death of one twin in-utero. The risk of cerebral palsy in the surviving twin where the other twin died in utero is 10-20 times greater. In summary, there is no single cause for the increased risk of cerebral palsy in multiple pregnancies, but is related to at least two major factors, prematurity and TTTS.

\section{P TWINS IN PSYCHOANALYTIC WORK}

\section{Vivienne J Lewin}

Correspondence address: 24 Estelle Road, London NW3 $2 J Y$.

Twins face particular developmental issues that are enacted within the transference relationship with an analyst/therapist. For each infant twin, the evolution of a personal identity involves separation from both twin and mother, as well as working through the Oedipal conflict - recognition of mother as the primary object, not the twin - and the parents as the generative couple, not the twin-couple. Where this three-person relationship can be established for each infant, boundaried by the relationship between the parents, an internal space is created, a capacity for observing and being observed - for thinking. The existence of a twin may hamper this, and an enmeshed twinship may be used as a haven 
to avoid these processes, leaving each infant with a sense of shared identity with the twin. A twin in analysis attempts to establish a twinship with the analyst in a psychic re-enactment of the actual twinship. This defensive twinning must be addressed or fundamental aspects of the personality will remain inadequately known and worked with, and development towards separateness and maturity will be impeded, with an unsuccessful ending to the work. Emotional growth involves facing loss and the pain of relinquishing phantasies of omnipotent contro so common in twinship. The wish may be to maintain a psychic retreat (the twinship) where this development is avoided. Work may be particularly difficult where one twin has died. Relinquishing the psychic twinship and mourning for the lost twin will enable the survivor to establish a more secure identity. The complex twin transference will be an important focus in psychoanalytic work and will need careful and detailed attention for a successful outcome to the work.

\section{S HERITABILITY FOR CANCER, COMPARISION BETWEEN TWIN AND FAMILY STUDIES.}

\section{Paul Lichtenstein}

Correspondence address: Department of Medical Epidemiology, Karolinska Institute Box 281, SE-171 77 Stockholm, Sweden. paul.lichtenstein@mep.ki.se

The relative importance of genes and environments for cancer susceptibility is not well understood. Twin studies have shown modest heritabilities ranging from $20 \%-40 \%$ of cancer susceptibility depending on site. Shared environmental effects have been of less importance and have explained between $0 \%-20 \%$ of susceptibility. Due to limited numbers of twins, those estimates have relative low precision. In this presentation we will compare results from twin studies with results from studies on nation-wide family data of all inhabitants in Sweden $(>6,000,000)$ since 1941. In general, family data mainly confirm the findings of moderate genetic effects. Those analyses also show significant shared environmental effects, both from shared adult environments and from shared childhood environments. Compared to twin studies, the family studies show somewhat lower heritability estimates and higher shared environmental effects. The difference might be that twin studies include nonadditive genetic effects, and that family studies might underestimate heritability due to age-dependent genetic effects. We conclude that twin and family studies agree in that environmental effects have the principal role in cancer development, and that these effects are mainly nonshared. The relative large genetic effects, accounting for around $20 \%-40 \%$ of susceptibility, indicated that even though susceptibility genes have been described at many cancer sites, they are likely to explain only part of the genetic effects.

\section{$143 S$ REPRODUCTIVE MEDICINE, MULTIPLE BIRTHS AND SEQUELAE FOR THE CHILDREN}

\section{Brian A Lieberman}

Correspondence address: Manchester Fertility Services, Russell House, Russell Road, Whalley Range, Manchester M16 8 A

The incidence of multiple births has increased due to medical treatments for the infertile couple. The rising incidence has been associated with increased demands on obstetric, neonatal and paediatric services. A number of strategies have been employed to prevent any further increase in the incidence of iatrogenic induced multiple pregnancy. None of these strategies will suffice except for the replacement of a single embryo or uni-follicular ovarian stimulation. The adverse effect on preg nancy and the resulting offspring will be analysed and discussed.

\section{$144 \mathrm{P}$}

\section{GENETIC AND ENVIRONMENTAL INFLUENCES ON SELF-REPORTED LIFE EVENTS IN WOMEN OF THE FINNISH TWIN COHORT}

Kirsi Lillberg,Pia K.Verkasalo, Jaakko Kaprio, Markku Koskenvuo.

Correspondence address: The Finnish Twin Cohort Study, Dept. of Public Health,

P.O. Box 41, FIN-00014 University of Helsinki, Finland.

Intrapersonal characteristics are suspected to play a role in the occurrence and reporting of life events. We investigated the extent to which genetic factors influence self-reports of life events in the last 5 years. The study was based on 4,044 Finnish female twin pairs born before 1958 who reported about the occurrence of 21 life events in a questionnaire in 1981. A subsample of the twins born between 1930-1957 answered a follow-up questionnaire in 1990. Structural equation modeling was used to estimate the genetic and environmental effects on liability to life events The median number of life events reported for the past 5 years was 4 (interquartile range 2-6). The best fitting genetic model was the ACE model indicating that genetic effects accounted for $29 \%(95 \%$ CI $18-39 \%)$ and shared environmental effects for $12 \%(4-20 \%)$ of the phenotypic variance of reports of life events. The magnitude of genetic effects was similar for younger (24-59 years) and older (60 years or more) women. We found genetic effects also for events considered to be independent of the individual's behavior. These preliminary results indicate moderate genetic influence on self-reports of recent life events in women, thereby confirming previous findings that life events are not random. We will also assess whether the genetic and environmental factors affecting life event reporting are stable over time.

\section{F WHY A NATIONAL PARENT AND USER GROUP IS ESSENTIAL TO IMPROVE THE QUALITY OF HEALTH AND SOCIAL CARE.}

\section{Judi Linney and David Stern}

Correspondence address: Twins and Multiple Births Association, 27 Woodham Park

Road, Woodham, Addlestone, Surrey

This presentation will outline a review that was undertaken to assess the views of members of a national organisation, Twins and Multiple Births Association. The aim of the project was to examine current service provision, needs and demands, and how these were going to be met in future. The number of multiple births are rising but many statutory services do not recognise the greater health risks nor address the specific needs of families with multiples. Areas identified included physical and emotional health of parents, the financial burden, lack of nursing and home help and social isolation. Additional needs were highlighted in families where there were triplets or more, a bereavement, a lone parent, or a child with a disability. These needs were often not recognised by professional carers. Education, training and information for parents and professionals were key recommendations. Parents and users must be more involved in planning and provision of services. Through working in partnership with parents and users, professionals can improve the quality of health and social care to families with twins, triplets or more.

\section{S ARE DISEASE DISCORDANT IDENTICAL TWINS THE PERFECT DEVICE TO STUDY AUTOIMMUNITY?}

\section{Marco Londei}

Correspondence address: Imperial College School of Medicine, Kennedy Institute

of Rheumatology Division, 1 Apenlea Rd W68LH, London.m.londei@ic.ac.uk

Autoimmune diseases are strongly controlled by genetic factors, the main being alleles of the HLA locus. Though HLA matched 'controls' can be found more difficult is the task to match all the genetic factors influencing autoimmunity. A perfect 'control' should therefore 'share' the same genetic make up but differing in the clinical manifestation. If genetic factors are at the foundation of autoimmunity the environmental ones represent the triggers of the pathology. Ideal 'controls', therefore, should also have experienced the same environment. Individuals who respond to these provisions are identical twins as they share the same genetic make-up, and normally share the same environment. Remarkably the concordance rate of autoimmune diseases in identical twins is not absolute and only a relatively small fraction is concordant. These disease 'discordant' identical twins thus represent the perfect group of patients and controls to study modifications of the immune system likely associated to the disease. It has to be stressed that normally identical twins have a very similar immune profile. For this purpose we have embarked in the analysis of different parameters both of the adaptive as well as of the innate immune system, in order to pinpoint difference between disease discordant identical twins. Results from these studies will be presented and their potential significance for the evolution, pathogenesis and potential therapy of the autoimmune disease discussed.

\section{$147 S$ THE FETAL ORIGINS OF ADULT DISEASE - A TWIN APPROACH}

Ruth JF Loos; Robert Fagard; Gaston Beunen; Catherine Derom; David Phillips; Robert Vlietinck.

Correspondence address: Centre for Human Genetics, Katholieke Universiteit Leuven, Kapucijnenvoer 33 blok j, B-3000 Leuven, Belgium

A large number of epidemiological studies have shown that low birthweight is associated with an increased risk of cardiovascular and metabolic disease in adult life. We have studied body composition and glucose-insulin metabolism in 424 twin pairs aged 25.8 (4.7) years, from the East Flanders Prospective Twin Survey. Twin members share the same maternal environment. Nevertheless, each fetus has its own feto-placental environment, which may differ substantially from that of the co-twin. Because of their genetic identity, differences between monozygotic (MZ) twins at birth can be attributed to the feto-placental environment. Pair-wise analysis showed that the lighter twin at birth was significantly smaller and lighter as an adult, but had a higher waist-tohip-ratio, more subcutaneous fat, and less lean body mass, compared to the heavier co-twin. These intra-pair differences increased with increasing intra-pair birthweight differences, in MZ as well as in dizygotic twins. Despite the large intra-pair birthweight difference, both MZ twins had similar fasting insulin and glucose concentrations. We also found that low maternal BMI before pregnancy and high maternal age at delivery were significantly associated with increased adult fasting 
insulin concentrations in the twin offspring. These studies suggest that maternal as well as feto-placental influences contribute to an adverse body habitus and to an abnormal glucose-insulin metabolism in adults. Studies of twins may shed light on the relative contribution of maternal and feto-placental influences to the programming of adult diseases.

\section{P OPPOSITE-SEX TWINS}

\section{Olivia Lousada}

Correspondence address: 14 Allerton Rd, London, N16 $5 \mathrm{UJ}$

This research was begun in response to professional and personal interest in the disquiet of opposite-sex twins. This disquiet is substantiated by psychological theory being ultimately based in the individual. The research, Increased Occurrence of Schizophrenia and other Psychiatric Illnesses by Ulla Klaning, Preben Bo Mortensen and Kirsten Ohm Kyvik (British Journal of Psychiatry (1996) 168, 688-692), found: "The rate ratio for any psychiatric disorder in twins from opposite-sex pairs was 1.29 times higher than the rate ratio in same sex twins. Likewise, the rate ratio for schizophrenia in twins from opposite-sex pairs was 1.77 times higher than the rate ratio in same sex twins."

The interviewing methods for this research are derived from the methods of Psychodrama and Sociometry and the theory of spontaneity invented by Jacob Levi Moreno, complementary to Action Research:

1. The twins make paintings of their experience of being a twin.

2. They make a map (Sociogram) showing what is important to them.

3. They role-reverse with significant others of their own choice to contemplate their place in the twin's and families' life.

The outcome of the research will not only be to compare the different work of each individual but compare the twins, and each pair to the others. Amongst the findings so far I have observed they are in caring or public roles, playing a significant, even unique role in the community, but often have difficulties with intimate relationships. They have high levels of anxiety and frustration. This presentation will show a small section of the method of work and how it illuminates the research.

\section{P PRENATAL INTERVENTIONS TO IMPROVE TWIN PREGNANCIES OUTCOMES}

\section{Barbara Luke, Linda Min, Ruta Misiunas, Clark Nugent}

Correspondence address: University of Michigan, Ann Arbor, Michigan, USA

Twin pregnancies are at higher risk for maternal and neonatal complications. The purpose of this study was to evaluate the effect of a program of prenatal diet therapy and patient education on reducing complications and improving outcomes. This was a prospective intervention study of women who participated in a special program (PP, program pregnancies), compared with non-participants (NPP, non-program pregnancies) and delivered between 5/96-8/00. Selection criteria for PP included enrollment in the program prior to 25 weeks and $\geq 4$ program visits; NPP had no program visits; all twin pairs were liveborn and without major anomalies. The study sample included 91 PP (182 infants) and 157 NPP (314 infants). Regression analyses indicated that PP mothers were less likely to experience preterm premature rupture of membranes (PPROM) (adjusted odds ratio $\{\mathrm{AOR}\} 0.23,95 \%$ Confidence Intervals $\{\mathrm{CI}$ $0.10-0.51, p=.0003$ ), preterm labor (AOR 0.58, CI 0.37-0.90, $p=.01$ ), early preterm delivery (<33 weeks) (AOR 0.46, CI $0.28-0.74, p=.001$ ) or to have infants who were $<1,500$ grams (AOR 0.09, CI 0.03-0.28, $p<.0001$ ) or $<2,500$ grams (AOR 0.43, CI 0.29-0.66, $p=.0001$ ). Program participation resulted in higher birthweight $(+225$ grams per twin, $p<.0001)$, longer gestation $(+5.75$ days, $p=.006)$, higher birthweight-for-gestation (zscore, $+0.24, p=.01$ ), shorter newborn length of stay $(-10.6$ days per twin pair, $p=.001)$, and lower total cost at birth for the mother and twins $(-\$ 36,142, p<.0001)$. These findings suggest that participation in a special prenatal program for mothers of twins was associated with significant reductions in maternal and neonatal complications, resulting in dramatic cost savings.

\section{P THE MENTAL AND MOTOR DEVELOPMENT OF TWINS: THE ROLE OF PLACENTATION}

Barbara Luke, Ruta Misiunas, Barbara Burpee,Shirley Gogliotti, Elaine Anderson Correspondence address: University of Michigan, Ann Arbor, Michigan, USA

As part of an ongoing prospective study of twins, follow-up evaluations were conducted using the Bayley Scales of Infant Development, the Infant Neurological International Battery, and the Peabody Developmental Motor Scales on 90 twins tested at 8-months, 77 twins tested at 18-months, and 34 twins tested at 3-years. The purpose of this study was to evaluate the mental and physical development of twin children by placentation (dichorionic, DC, vs monochorionic, MC). Logistic and linear regression modeling was used to evaluate the effects of placentation. MC twins had a significantly slower rate of fetal growth $(-2.2 \mathrm{~g} /$ week to 20 weeks, $p=.002 ;-9.8 \mathrm{~g} /$ week from $20-28$ weeks, $p<.0001 ;-19.3 \mathrm{~g} /$ week from 28 weeks to birth, $p<.0001)$, shorter gestations $(-1.4$ weeks, $p<.0001)$, lower birthweights $(-397 \mathrm{~g}, p<.0001)$, shorter birthlengths $(-2.8 \mathrm{~cm}, p<.0001)$, but comparable head circumferences compared to DC twins. At 8 months of age MC twins averaged lower mental scores $(-8.44, p=.001)$ and motor scores $(-13.9, p<.0001)$ and were more likely to be rated as mildly or significantly delayed (OR 7.89, CI 3.23-19.40). For children tested at 18-months and 3-years, no significant differences were noted. Overall, MC children were more likely to be rated as mildly or significantly delayed (OR 6.41, CI 2.97-13.82). These findings suggest that $\mathrm{MC}$ twins are at higher risk for mental and motor delays, particularly during infancy, and highlight the importance of early childhood screening and interventions for these high-risk children.

\section{F THE SPECIFICITY OF FAMILIAL RISK FOR MANIA VERSUS ALCOHOLISM}

Courtney E. Lynch, Michael J. Lyons, Beth A. Jerskey, Denise A. Hines,

Simone Ascher and Ming Tsuang

Correspondence address: Boston University, 64 Cummington Street, Boston, MA 02215

Epidemiologic data have shown that bipolar disorder has a high rate of comorbidity with substance abuse. We examined data from male twin pairs to determine whether bipolar disorder and substance abuse share a common vulnerability or if having one disorder is a risk factor for developing the other disorder. Data were collected from members of the Vietnam Era Veteran Twin Registry. The Registry comprises male twin pairs who served in the U.S. military between 1965 and 1975. In 1991 approximately 8,000 twins were interviewed by telephone using the Diagnostic Interview Schedule to diagnose mental disorders. We defined mania as the presence of three or more symptoms of a manic episode and alcoholism was defined as the presence of alcohol dependence, both according to DSM-III-R criteria. Within individuals, there was a strong association between mania and alcoholism. To compare across twin pairs, twins were divided into four mutually exclusive groups: 1) no mania, no alcoholism; 2) mania, no alcoholism; 3) no mania, alcoholism; and 4) mania and alcoholism. Odds ratios were used to quantify the cross-twin associations. Mania in one twin was associated with an increased risk of mania in the co-twin, and alcoholism in one twin was associated with an elevated risk of alcoholism in the co-twin. However, mania alone in one twin was not associated with alcoholism alone in the co-twin and alcoholism alone in one twin was not associated with mania alone in the co-twin. Thus, results indicate specificity of familial risk for alcoholism versus mania.

\section{$152 \mathrm{~F}$}

\section{"SELF-MEDICATION" WITH NICOTINE:} TWIN PAIRS DISCORDANT FOR SCHIZOPHRENIA

Michael J. Lyons, Beth A. Jerskey, Courtney E. Lynch, Hong Xian, Jessica Bar and Ming Tsuang

Correspondence address: Boston University, 64 Cummington Street, Boston, MA 02215

Clinical observation and previous research has identified a relationship between schizophrenia and cigarette smoking. It has been suggested that nicotine use may represent "self-medication" on the part of individuals with schizophrenia; nicotine may help to normalize cognitive processes such as attention. We studied 24 pairs of male twins from the Vietnam Era Twin Registry in which one twin received a hospital diagnosis of schizophrenia and his co-twin did not. These schizophrenia-discordant pairs were compared on smoking related variables to twins from approximately 3,000 pairs in which neither twin had schizophrenia. Compared to controls, the co-twins of schizophrenics were significantly more likely to have smoked. Both schizophrenics and their co-twins were significantly more likely to report the inability to quit smoking than controls. The co-twins of schizophrenics were significantly more likely than controls to report each of the following symptoms when they tried to quit: difficulty concentrating, nervousness, headache, and drowsiness. They did not differ significantly from controls on the following symptoms: irritability, craving, upset stomach, increased appetite, and hands shaking. There results suggest that individuals at "high-risk" for schizophrenia by virtue of sharing genes with a schizophrenic individual demonstrate a pattern consistent with the use of nicotine to remediate attentional difficulties.

\section{$153 S$ ONE AND ONE MAKE(S) THREE: A GROUNDED THEORY STUDY OF TWINSHIP AND THE NEEDS OF TWINS IN PSYCHOLOGICAL COUNSELLING.}

Alison M Macdonald

Correspondence address: Department of Psychology, City University, Northampton Square, London EC1V OHB

The primary foci of twin research have been on twins as a biological phenomenon, the management of medical complications of twin pregnancy and birth, and by psychologists and other scientists for whom twins provide a powerful research design for the study of individual differences 
and development. Twin relationships, or twinships, have received less research attention. Most therapeutic models are based on the experience and development of singletons. This qualititative study used an adapted form of grounded theory methodology to explore the nature of twinship and, in particular, issues of relevance in psychological counselling with twins. Fourteen participants were interviewed in depth about their understanding and experience of twinship and factors that may be important in psychological counselling. Purposive sampling was used to select participants with a rich knowledge and experience of twinship and therapeutic work. Constant comparison of segments of transcribed interviews was used to describe and elaborate concepts in the data that were found to be repeated within and between participants. A core theme, discernment, was found to be a central task of twinship, and for others in relation to twins. It is the process whereby twins are 'made separate', and happens at many levels of meaning. Four main subcategories are subsumed by the core category, including 'two at the same time', 'awareness', 'managing ambiguity' and 'defining boundaries' Implications for psychological counselling with twins are discussed.

\section{$154 \mathrm{~S}$}

\section{THE USE OF CLUSTER ANALYSIS IN MULTIVARIATE MODELS}

\section{Alex MacGregor}

Correspondence address: Twin Research and Genetic Epidemiology Unit, St Thomas Hospital, London, UK SE1 7EH

The search for the genetic basis of complex disease is potentially impeded by the lack of direct correspondence between the clinically recognised manifestations of disease and the action of specific genes. The problem is illustrated in osteoarthritis (OA). The disease is defined clinically through the recognition of a collection of features identified on X-ray (joint space narrowing, osteophyte, deformity and cyst formation) in an individual joint. Yet quite separate genetic influences are likely to determine each of these individual components, as well as the pattern of involvement at different body sites, the timing of disease onset and the rate of progression. Studying twins provides an efficient method of identifying the existence of shared and specific genetic effects in phenotypic data. Available methodology is limited for screening phenotypes like OA that comprise potentially large sets of variables, with sparse and categorical data types. One solution is provided by multivariate scaling methods that use a distance matrix derived from the phenotypic crosscorrelations of contrasting zygosities. This approach has been used to examine the underlying genetic basis of $\mathrm{OA}$, thus providing a coherent model of disease for standard genetic linkage and association studies.

\section{$155 \mathrm{P}$}

GENETIC FACTORS DETERMINE THE LIABILITY
TO BACK PAIN REPORTING: RESULTS
OF A POPULATION BASED MRI STUDY OF TWINS

\section{AJ MacGregor,T Andrew, P Sambrook, TD Spector}

Correspondence address: Twin Research and Genetic Epidemiology Unit, St Thomas Hospital, London, UK SE1 7EH

Objective. We examined data from a population sample of female twins to assess the extent to which genetic factors contribute to the reporting of pain and examine the relative contribution of structural changes documented on MRI, and of behavioural and other lifestyle variables.

Methods. Questionnaire data relating to the lifetime history of back pain were examined from 181 monozygotic (MZ) and 351 dizygotic (DZ) female twin pairs. Lumbar MRI scans were available in a representative sample of $81 \mathrm{MZ}$ and $174 \mathrm{DZ}$ twin pairs and were scored for disc degeneration. The data were analysed using structural equation modelling.

Results. The lifetime cumulative prevalence of clinically relevant high intensity pain was $18 \%$. Contrasting MZ and DZ concordances showed a significant contribution from genetic factors, equating to a heritability of $57 \%(95 \% \mathrm{CI}:\{35 \%, 78 \%\})$. Significant associations $(p<0.05)$ were observed between back pain and the extent of MRI change, GHQ score, body mass index and smoking history. Genetic factors determining MRI changes accounted for $10 \%$ of the genetic variance in back pain; $7 \%$ was explained by genes in common with GHQ score.

Conclusion. Genetic factors explain the majority of the variance in liability to back pain in the female population. These results suggest that future research into the causes of back pain should include identifying the genes that determine structural degenerative changes in the spine and behavioural responses to painful stimuli.
Alex J MacGregor, Lynn F Cherkas, Tim D Spector

Correspondence address: Twin Research and Genetic Epidemiology Unit, St Thomas Hospital, Lambeth Palace Road, London SE1 7EH

Objective. The occurrence of Raynaud's Phenomenon (RP) may be a reflection of a generalised tendency to abnormal vascular responsiveness, other manifestations of which may include migraine, angina and pulmonary hypertension. RP itself has a strong genetic basis. In this study we examine the co-occurrence of RP and migraine in a population sample of twins to assess if there is a common underlying genetic basis to these two conditions.

Methods. Questionnaires were sent to 3,652 female twins asking about digital colour changes in response to the cold and headache history. Replies were received from 702 identical (MZ) and 727 non-identical (DZ) pairs (mean age 48 years). RP and migraine were classified using standard clinical criteria. Variance componen ts analysis was used to assess the genetic contribution to both traits individually and the extent to which genetic and environmental factors accounted for their association.

Results. RP 'ever' occurred in $11 \%$ and migraine $24 \%$. The heritability of RP was $56 \%$ and of migraine was $41 \%$. Subjects with RP were at 2 -fold increased risk of having migraine $(p<0.05)$. The association could be accounted for entirely by genetic factors. Shared genetic factors accounted for only $10 \%$ of the overall genetic variance in both traits.

Conclusion. The data support a common genetic basis for the pathological changes that occur in RP and migraine.

\section{F PHENOTYPE OF HERITABLE BREAST CANCER ACCORDING TO THE OCCURRENCE IN TWINS}

Thomas M. Mack Ann S. Hamilton, \& Edward B. Rappaport

Correspondence address: USC Cancer Center, 1441 Eastlake Ave, Los Angeles CA

Background. The pattern of heritable breast cancer as a class has not been well described, and the predominate phenotype is a matter of speculation. The incidence among identical twins of breast cancer cases reflects heritable as well as non-heritable cancer occurrence.

Methods. We prospectively followed the female co-twins of 6325 female twins with cancer and compared the frequency of new breast cancer cases to that expected.

Results. While co-twins of other cancer probands are at little increased risk of breast or other cancer generally, and risk to fraternal co-twins of breast cancer cases is similar to that of other siblings $(\mathrm{SIR}=1.7, \mathrm{CI}=$ 1.1-2.6), risk to identical co-twins of cases is much higher ( $\operatorname{SIR}=4.4$, $\mathrm{CI}=3.6-5.6)$. Moreover, the incidence rate among identical co-twins of cases maintains a constant high rate with age. Concordant diagnoses in young twin pairs cluster non-randomly over time.

Conclusions. The high and constant heritable breast cancer incidence cannot be explained by cumulative estrogen exposure. The contrast in SIR between fraternal and identical co-twins is too large to be produced by single autosomal dominant mutations, and rather suggests multiple coexisting genomic mutations. The phenotype may reflect heightened susceptibility to hormones, a defect in tumor suppression, or both. This allotype is highly penetrant and probably accounts for a substantial proportion of all cases.

\section{F GENETIC EPIDEMIOLOGY OF CARDIOVASCULAR REACTIVITY IN ADOLESCENCE}

Hermine H Maes, Michael C Neale, Lindon J Eaves, Richard M Schieken Virginia Institute for Psychiatric \& Behavioral Genetics, Virginia Commonwealth University, Richmond. Supported by HL60688 and MH57761

Background. Previous studies of genetic factors in cardiovascular reactivity suffered from small sample size and yielded inconsistent results. Here we report results from the largest study of genetic factors in cardiovascular reactivity in adolescence.

Aims. To test whether: (i) family resemblance for cardiovascular reactivity is due to genetic or shared environmental factors; (ii) the same genetic factors contribute significantly during physical and/or mental stress.

Methods. Blood pressure data were collected at rest and during bike exercise, handgrip and arithmetic exercise in 317 white twin pairs between the ages of 9.5 and 17 from the MCV Cardiovascular Twin Study. We calculated the response of blood pressure to stress under 3 different conditions by taking the difference between maximal and resting blood pressure. Structural equation models were fitted to partition the variance in the contribution of genetic, shared and unique environmental factors.

Results. Familial resemblance was significant for blood pressure reactivity, most likely explained by additive genetic factors with heritabilities ranging between 10 and $40 \%$. Both testing whether genetic factors 
are independent or in common for variability in blood pressure response to 3 stress situations resulted in significant loss of fit. Sex differences in the contribution of genetic and environmental factors were significant.

Discussion. We conclude that additive genetic factors likely account for family resemblance for cardiovascular reactivity and that partly the same and partly different genetic factors account for reactivity to physical and mental stress. These results suggest that molecular genetic studies will find different genes for resting blood pressure and reactivity.

\section{P GENETIC AND ENVIRONMENTAL INFLUENCES ON} SELF PERCEPTIONS IN CHILDHOOD AND PUBERTY

Kazumi Maeshiro (Shirayuri College), Masumi Sugawara (National Institute of Mental Health, Japan),Atsushi Sakai (Waseda University), Sachiko Amou (Aoyama Institute of Education)

Correspondence address: Shirayuri College, 1-25 Midorigaoka,

Choufu, Tokyo, 182-8525, Japan

The purpose of this study was to investigate the magnitudes of genetic and environmental influences on self-perception.

As part of ongoing longitudinal study in a Japanese twin sample (MZ and DZ, 0-15 years old, total 2,135 pairs), 381 twin pairs (215 MZ pairs, 166 DZ pairs) in childhood and puberty completed Harter's 1985 Self-Perception Profile for Children scale. This version contains 6 subscales including Scholastic, Social acceptance, Athletic, Physical appearance, Behavioural conduct as well as Global self-worth. Except for Behavioral conduct, the internal consistency reliabilities were accept able range $(\alpha=.75 \sim .84)$. So this study used 5 subscales.

Main results were followed. First, MZ twins interclass correlations exceeded DZ twins for all subscales. Second, using genetic ACE (Additive genetic, Common environment, and non-shared Environment) model, the results indicated significant Additive genetic influence for Scholastic, Physical appearance and Global self-worth. Social acceptance, Athletic, Physical appearance and Global self-worth were influenced by Common environment, on the other hands, all subscales were influenced by non-shared Environment.

\section{P DOES ZYGOSITY AND SEX IN TWIN PAIRS INFLUENCE RISK OF PRE-ECLAMPSIA IN THE MOTHER?}

Per Magnus, Camilla Stoltenberg, Lill Trogstad,Anne Eskild, Kristian Tambs, and Jennifer R. Harris

Correspondence address: The National Institute of Public Health, Oslo, Norway

The number of different paternal genes is larger in dizygotic (DZ) than in monozygotic (MZ) twin pregnancies and singleton pregnancies. Based on the hypothesis that pre-eclampsia is caused by maternal reactions to paternally derived foetal genes, we expected an increased risk of pre-eclampsia among mothers carrying DZ versus MZ twins.

The hypothesis was tested by comparing the risk of pre-eclampsia in 5,232 mothers carrying twins with known zygosity. These twins were part of a population based twin registry nested within the national birth registry for Norway 1967-1997. Further analysis based on data about 17,517 mothers of twins in the birth registry explored the effect of the sex of the twin pair on the risk of pre-eclampsia. Relative risks were estimated as odds ratios (OR) with $95 \%$ confidence intervals, adjusted for parity, maternal age, and twins' year of birth.

Zygosity was not associated to risk of pre-eclampsia. The relative risk was $1.00(0.82-1.20)$ for DZ twins. However, the number of females in the twin pair tended to increase the risk of pre-eclampsia in the mother. Relative to pregnancies with two male foetuses, the risk was 1.09 $(0.96-1.24)$ for pairs with one female, and $1.12(0.99-1.27)$ for twin pairs with two females.

The increased number of different paternally derived foetal genes in DZ twins does not increase the risk of pre-eclampsia in the mother. A slightly increased risk in female twin pregnancies suggests that hormonal levels or other effects of sex chromosomes play a role in the aetiology of pre-eclampsia.

\section{P TWINS AND LEISURE TIME PHYSICAL ACTIVITY. A UNIVARIATE STUDY}

José A.R. Maia, Martine Thomis, Gaston Beunen, Vitor Lopes

Correspondence address: Faculty of Sport Sciences, University of Porto, Portugal

The purpose of this study was to evaluate the magnitude of individual differences in leisure time physical activity conditional on genetic differences using the classic twin method. Total sample size is composed of 411 twin pairs of different zygosity: MZ males, $n=85$; MZ females, $n=118$; DZ males, $n=68$; DZ females, $n=85$; DZ opposite sex, $n=55$. Zygosity was evaluated according to the indirect method of Peeters et al., (1998), and leisure time physical activity was assessed with the Baecke questionnaire (Baecke et al., 1982). Quantitative genetic modeling was done with Mx software (Neale, 1997). Formal testing was conducted in several alternative models for additive genes (A), common (C) and unique environment (E). The correlation pattern suggests different effects of genes and environment The best fitting model ACE/female-AE/male suggests specific contribution of latent factors: $\chi^{2}(10)=6.32, p=0.79$; AIC $=-13.68$; RMSEA $=0.02$. Major effects for each gender are as follows: males, $\mathrm{A}=63 \% ; \mathrm{E}=37 \%$; females, $\mathrm{A}=32 \%, \mathrm{C}=38 \%, \mathrm{E}=30 \%$.

Conclusions. (1) there are significant genetic effects in variation of leisure time physical activity levels; (2) these effects are of different importance in males and in females; (3) the presence of environmental effects in females but not in males has been previously noted in family aggregation studies.

\section{S BLOOD FLOW CHANGES IN DISCORDANT TWINS.}

Peter Malcus, Lene Sperling, Ricardo Laurini

Correspondence address: Department of Obstetrics and Gynaecology, University Hospital of Lund, S-221 85 Lund, Sweden.

In the past two decades Doppler ultrasound assessment of high risk pregnancies have been accepted a valuable tool for evaluating fetal condition. Twin pregnancy is high risk and the perinatal mortality rate in twins is six times higher than among singletons, mainly due to prematurity. It is also three to four times higher in monochorionic compared with dichorionic twins. Approximately $2 / 3$ of twins are dichorionic and $1 / 3$ is monochorionic. Membranes thicker than $2 \mathrm{~mm}$ and the presence of the lambda sign of membrane insertion in diamniotic twins, in the first trimester, indicate dichorionicity. Fetal growth in twins is more difficult to assess than in singletons since ultrasound growth charts available are made up for singletons only. The appearance of umbilical artery blood flow waveform changes is related to increased placental vascular resistance correlated to histopathological changes. These may be the same in singleton pegnancies with placenta insufficiency as in twins with discordant growth. Doppler studies can therefore give important information about placenta insufficiency also in mono- and dichorionic twins with discordant growth. In monochorionic twins with discordant growth and without umbilical artery waveform changes twin to twin transfusion should be suspected. Uncontrolled as well as randomised controlled Doppler studies in twin pregnancies have shown significant decrease in perinatal deaths in the Doppler group compared with controls. We report our blood flow results and placenta morphology in dichorionic twin pregnancies with discordant growth.

\section{NAVIGATING THE CULTURAL UNIVERSE OF GENETIC RESEARCH AMONG AMERICAN INDIANS}

\section{Spero Manson, Paul Spicer.}

Correspondence address: American Indian and Alaska Native Programs, Psychiatry.

UCHSC, 4455 E. 12th Ave, Denver, CO, 80220

Recent attempts to extend the NIH Human Genome Project to include ethnic minority populations led to an outcry regarding ethical, legal, social, and political issues in genetics research among these segments of U.S. society. American Indian communities have been particularly vocal, concerned that, without local controls, the process, materials, and outcomes of this work will violate matters of culture and self-determination. Consequently, important genetic research is stalled, and awaits the resolution of such tensions. Focusing on the American Indian experience, we review the historical events that precipitated this impasse and highlight specific concerns that have emerged, with special emphasis on studies of psychopathology. Several codes of conduct and collaborative agreements negotiated between investigators and tribal communities are described as a means of illustrating the principles likely to characterize future partnerships required to advance this line of inquiry. These principles revolve around answers to questions like: What can genetic research contribute to addressing the mental health problems of greatest concern to Native people? What are the risks and benefits to individual members, as well as tribal communities in general, by participating in this research? What rights can Indian and Native people and tribal communities have over biological materials used in such research? What is the authority of tribes, as domestic dependent sovereignties, to regulate genetic research and materials? What institutions can tribes and Indian and Native communities put in place to protect themselves from the risks and to ensure that they participate fully in the benefits of genetic research?

\section{$164 S$ DOUBLE BIND: ANOREXIA NERVOSA IN MONOZYGOTIC TWINS}

\section{Patricia Marsden}

Correspondence address: Eating Disorders Research Team, Dept of Psychiatry,

St George's Hospital Medical School, Cranmer Terrace, London SW17 ORE.

This case study of four women patients - two pairs of monozygotic twins - considers the ways in which the experience of twinship may relate to the development of Anorexia Nervosa. It is suggested that, 
whilst all infants face the task of separation-individu ation from their primary care-giver, twins face a dual challenge in that they also need to negotiate a process of separation-individuation from the other twin. It is further suggested that this task presents particular difficulties for monozygotic twins. Anorexia Nervosa may represent an attempt to avoid separation by means of a retreat to a pre-adolescent state in which differences, envy and rivalry can be denied. Successful treatment will need to provide the opportunity for each patient to address her difficulties without constant reference to the other and admission to separate hospitals seems to be helpful in this.

\section{$165 \mathrm{P}$}

\section{THE MATCHED MULTIPLE BIRTH DATA SET: A NEW DATA FILE FROM THE U.S. NATIONAL CENTER FOR HEALTH STATISTICS}

Joyce A. Martin, Sally A. Curtin, Kenneth G. Keppel, M. Louise Saulnier The National Center for Health Statistics, Room 810, 6525 Belcrest Rd. Hyattsville, MD 20782

In the United States between 1980 and 1998, the number of twin births climbed $62 \%$, and triplet/ + births by $470 \%$. In $1998,30 \%$ of all very low birthweight infants, and $18 \%$ of all neonatal deaths were born in multiple deliveries. Despite their growing numbers and the substantial impact multiple births are exerting on measures of infant health, the U.S has lacked a national data base with information on sets of multiples. This report describes the first comprehensive U.S. data file to include matched sets of births and fetal deaths in multiple deliveries. Selected research results will also be presented. "The Matched Multiple Birth Data Set" was created to fill a gap in U.S. natality and fetal death files. These files, based on birth and death certificate data, contain important data on individual events, but lack information on multiple sets; set mates are not linked together in any way. As a result, it has not been possible to examine basic characteristics of the multiple set such as gender-type and birthweight differences among set mates. The new data file includes a wealth of information on sets of twin and triplets born to U.S. residents for 1995-97. The vast majority $(98.8 \%)$ of all twin and triplet records were matched for a total of 152,222 sets of twins and 5,353 sets of triplets. Corresponding linked infant deaths are also included.

\section{S FINDING GENES FOR COMMON DISEASES}

\section{Nicholas G. Martin}

Correspondence address: Queensland Institute of Medical Research, Brisbane, Australia

The genomics revolution offers the opportunity to isolate genes contributing to risk of complex diseases which are known to run in families but have no simple mode of inheritance. This has been made possible by collection of disease information on very large samples of families, the availability of large numbers of genetic markers which can be typed semi-automatically, and improved statistical and computing tools for analysis. The first step is genetic linkage analysis which looks for congruent patterns of transmission of genetic markers and disease occurrence within families. Sibling pairs are particularly useful since they are common and minimise heterogeneity, and DZ twins are optimal since they minimise genotype $\mathrm{x}$ age interaction. Progression from linkage to isolating the gene will be facilitated by availability of the full human DNA sequence. Methods will be illustrated from searches for genes influencing melanoma, anxiety, alcoholism and other complex diseases.

\section{F EQUITY ISSUES AND EDUCATIONAL CONCERNS OF TWIN SIBLINGS}

\section{John R. Mascazine}

Correspondence address: P.O. Box 21852, Columbus, $\mathrm{OH} 43221$, USA mascazine.1@osu.edu

One hundred seventeen monozygotic twin pairs were interviewed and surveyed concerning their school and non-formal school experiences. The results indicated that many twin siblings had verifiable and legitimate concerns regarding how they were treated in learning situations.

Twins enter school and encounter many situations where they are treated very differently from single-born individuals by peers, teachers, and administrative personnel. Such treatment may sometimes lead to inequities and educational disadvantages. Studie s conducted in Australia, the United Kingdom, and the United States have indicated that indeed twins face many situations in their learning experiences that deserve attention and understanding of educators.

The themes of unequal treatment of twins in learning situations will be explained. Some of these themes include: the belief that the academic pursuits and academic performance of twin siblings are often similar, the expectation that twin siblings should / do compete academically, the idea that twins who share similar personality traits also share similar learning styles, and the notion that fair and equal treatment of each twin sibling is always in their best interest. These themes will be discussed in light of anecdotal evidence collected from interviews, open-ended surveys, twin learning style profiles, and artifact collection.

\section{John R. Mascazine}

Correspondence address: P. O. Box 21852, Columbus, OH 43221 USA (614) 793.9989

mascazine.1@osu.edu

This study compared the learning styles of monozygotic twins. It included analyzing perceptual, processing, and output modes when one attempts to learn new or difficult information. The reliable Dunn, Dunn, and Price learning style inventory was employed while qualitative data was gathered using open-ended surveys and interviews. The methods enabled the researcher to gather a broad and detailed array of data on the particular learning characteristics of twin siblings. For the 117 pairs of identical twins studied patterns emerged that were consistently supported by follow-up interviews and open-ended surveys. Data indicated that identical twin siblings do not share similar learning styles, and often develop quite different strategies when completing learning tasks. Even among the perceptual modalitie s of auditory, visual, tactual, and kinesthetic elements, identical twins rarely possessed the same modality preferences. Many presumptions about twin siblings and how they learn are incorrect or incomplete. The data could help parents, teachers, and school administrators understand twins as learners. Twins often reported having to cope with unique issues in their formal and informal learning experiences. Among these issues were: 1 . The role of competition among twin siblings in learning situations; 2 . The need for individual identity and recognition in learning situations; 3 . The positive impact that twin siblings had upon each other's educational experiences, and; 4 . The twins who were successful as learners were able to identify and utilize their individual learning style strengths.

\section{P HYPERPHENYLALANINEMIA IN MALE-MALE TWINS}

\section{Sophia Maximova Medeia Mkheidze}

Correspondence address: Municipal Centre for Human Genetics, Medical Academy for Postgraduate Training, St.Petersburg, Russian Federation

We report on a family with male-male MZ twins with hyperphenylalaninemia. Probands, MP and AP, 22-months-old boys, were born to a 32-year-old mother and a 33-year-old father (mother's 2nd pregnancy). This nonconsanguineous couple has an older healthy son 9 years aged. The mother suffers from myopia of high degree. The multiple pregnancy was complicated by a threatened miscarriage repeatedly and the delivery was performed by Cesarean section. At birth MP weight was $2750 \mathrm{~g}$, his length was $49 \mathrm{~cm}$; AP weight was $2200 \mathrm{~g}$, his length was $46 \mathrm{~cm}$. The probands were detected by routine newborn screening for hyperphenylalaninemia using dried blood spots: phenylalanine level (PheL, $\mu \mathrm{mol} / \mathrm{L}$ ) for MP was 291; 254, that for AP was 127; 206 (cut-off point is $122 \mu \mathrm{mol} / \mathrm{L}$ for newborns of St.Petersburg). The mother has 67 of PheL and the father has 73 of PheL. At the age 10 months MP weight was 9450 $\mathrm{g}$, his length was $71,5 \mathrm{~cm}$ and head circumstance was $43,5 \mathrm{~cm}$, PheL were $170 ; 236$. By this time AP weight was $9300 \mathrm{~g}$, his length was $71 \mathrm{~cm}$, head circumstance was $44 \mathrm{~cm}$ and PheL were 176; 303. Using HRLH method we were able to determine high PheL for AP proband $(748 \mu \mathrm{mol} / \mathrm{L}$, mild PKU). Molecular analysis was performed in the Laboratory for prenatal diagnosis of the Institute of obstetrics and gynecology named after D.O.Ott. Allele 1 of the twin-probands, their older brother and their mother was found to be R408W, the most wide spread mutation among Russian population. Allele 2 is not determined. Haplotypes of VNTR are different between older brother and twins.

\section{P MANAGEMENT AND OUTCOME OF TRIPLET PREGNANCIES RELATED TO ORIGIN AND CHORIONICITY}

Ashley Merién, Jim van Eyck, Bob Leerentveld, BirgitArabin

Correspondence address: Clara Angela Foundation Zwolle $(N L)$ \& Isala Clinics Dr.v.Heesweg 2 8025AB Zwolle (NL)

Objectives. Aim of the study was to evaluate management and outcome of triplet pregnancies related to origin and chorionicity.

Methods. Data of 35 triplet pregnancies born at our center from 1994-2000 were evaluated including 9 transported patients.

Results. The rate of TC and DC triplet pregnancies was 18/35 (51\%) and $17 / 35(49 \%)$ respectively; $24 / 35$ were induced by IVF, ICSI or ovulation induction; 4/5 triplets after ovulation induction were TC, 1 was DCTA. Among 16 IVF-triplets 10 were TC, 5 DCTA and 1 was DCDA. 9/11 spontaneous pregnancies were DCTA, 1 was DCDA and 1 TC. 3/17 DC triplet pregnancies revealed TTTS, all 2 DCDA pregnancies cord entanglement. The Cesarean section $(C S)$ rate was $24 / 35(68.6 \%)$, whereby $15 / 17(88 \%)$ of the DC but only $9 / 18(50 \%)$ of the TC pregnancies. Among the 9 vaginal deliveries, 7 were TC and 2 were DC. In two cases, delayed interval delivery was performed with a vaginal delivery and later CS of the remaining triplets. The perinatal mortality rate was $12 / 105(11.4 \%), 3$ triplets died in utero, due to hydrops (1) or placental insufficiency(2); 7 died post partum due to immaturity (5) or diaphrag- 
matic hernia (2 MZ). The prematurity rate $<32$ weeks was 14/35 (40\%), $8 / 26(31 \%)$ of pregnancies followed at our unit, however 6/9 (66\%) of transport pregnancies, $29 \%$ (7/24) of induced triplet pregnancies compared to $64 \%$ (7/11) of spontaneous triplets were born $<32$ weeks.

Conclusions. Chorionicity is influenced by origin of pregnancy and has a significant impact on management and outcome. The rate of prematurity might as well be influenced by prenatal care. TTTS or entanglement also occur in DC triplet pregnancies.

\section{P GENETIC AND ENVIRONMENTAL INFLUENCES ON LIPIDS, LIPOPROTEINS AND APOLIPOPROTEINS: EFFECTS OF THE MENOPAUSE}

\section{Rita P.S. Middelberg',Tim D. Spector ${ }^{2}$, Harold Snieder ${ }^{2}$}

Correspondence address: ${ }^{\prime}$ Gemini Genomics, Cambridge, UK. ${ }^{2}$ Twin Research

and Genetic Epidemiology Unit, St. Thomas' Hospital, London, UK.

Levels of lipids and (apo)lipoproteins are known to increase after the menopause, but it is unknown whether the magnitude of the genetic and environmental variance components changes with the menopause and whether lipids and (apo)lipoprote ins are influenced by different genes before and after the menopause. We studied 453 monozygotic and 1280 dizygotic pairs of female Caucasian twins (mean age: 48 yrs range: 18-79) from the St. Thomas' UK Adult Twin Registry. The measured serum lipids and (apo)lipoproteins included: total cholesterol, low density lipoprotein (LDL), high density lipoprotein (HDL), lipoprotein(a) $\{\mathrm{Lp}(\mathrm{a})\}$, apolipoprotein A-I (apoA1) and B (apoB), and trigylcerides. Mean values were adjusted for age, menopause, fasting statu and hormone-replacement therapy using multiple regression. The genetic and environmental influences on serum lipid levels were estimated in pre- and post-menopausal women separately using a 6 group analysis (pre/pre, pre/post, post/post in MZ and DZ pairs) in Mx. Total variance showed an increase in triglycerides, HDL and apoB. Genetic influences on lipid and (apo)lipoprotein levels \{except for apoB and $\mathrm{Lp}(\mathrm{a})\}$ increase after the menopause, whereas shared environmental influences in tota cholesterol, LDL, HDL, apoA and apoB tend to decrease or even disappear (pre: $22 \%-34 \%$ vs post: $0 \%-23 \%$ ). No influence of the menopause on $\mathrm{Lp}$ (a) was found. Lipids were not influenced by different genes before and after the menopause.

\section{P THE NAMING OF TWINS}

\section{Medeia Mkheidze}

Correspondence address: The Medical Academy for Postgraduate Training, St.Petersburg, Russia.mmkheidze@yahoo.com

The naming of twin babies is an important and very difficult task for their parents. Their relatives, friends and medical staff can refer to the babies as individuals right from the start. We analyzed names of 526 random twins pairs: female-female pairs (199), male-male pairs (178) and malefemale pairs (149) born in 1963, 1965, 1969 and 1970 and compared these data with those for general population using information from the book "About Russian names" by A. Suslova and A. Superanskaya (Leningrad, 1991)

Results. Thirty-nine of male names and twenty of female names were used for our twin cohort. It means that 12 of boys and 26 of girls had a chance to have the same name. The choice of names for women was more limited then that for men. The lists of the wide spread male and female names were similar for general population and for our twins group. Alexander, Sergius, Alexis, Dmitrii, Andrew were registered for 43.2 per cent of the boys. Elena, Irene, Olga, Svetlana, Natalie, Tatiana, Marina were registered for 65.8 per cent of the girls. Fifteen of the different name pairs were found among 29.2 per cent of male-male twins. Seventeen of the different name pairs were registered for 43.7 per cent of female-female twins. Eight of different name pairs were found among 22.1per cent of female-male twins. In addition, 13.4 per cent of female-male twins, 12.9 per cent of male-male twins and 8 per cent of female-female twins were given twin-sounding names (total 11.2\%).

\section{P THE ANGIOTENSIN CONVERTING-ENZYME (ACE) GENEPOLYMORPHISM, TWINS AND PACTICAL GENETIC COUNSELLING}

Medeia O. Mkheidze, Natalia A.Gladkova,Vasily I. Kazakov, Igor B. Nazarov Correspondence address: Kamennoostrovskyi prospect 47-18, MAPT Code 197022 City St.Petersburg Country Russian Federation mmkheidze@yahoo.com.

The ACE gene polymorphism (ACEGP) is due to the insertion/deletion (I/D) of 286 bp of intron 16.Our aim was to determine ACE genotype and discuss possible genotype-disease association as a form of councelling of families with twins. Materials and methods: We studied 5 healthy MZ sister pairs selected among 73 families with twins pairs. For making a diagnosis of zygosity we used self-report survey methodology and information about any cases of cardiovascular disorders among relatives of twins was included in the questionnaire. ACEGP was determined by PCR with DNA extracted from buccal epithelium cells as matrix. The product of amplification was analyzed by $8 \%$ PAAG electrophoresis and visualized with ethidium bromide.

Results. All five of MZ female twins had DD genotype. We created special certificate in which a genotype of a proband was fixed, possible complications and predisposition to diseases were listed and recommendation of medicine treatment was done. The investigation of ACEGP in conjunction with the pedigree analysis creates conditions for early diagnosis, correct medicine treatment, prevention of complications as an important part of practical genetic counselling of twin cohort.

\section{F INTER-TWIN RELATIONSHIPS AND MENTAL HEALTH}

Irma Moilanen, Eija Siikala, Sari Tamppari

Correspondence address: Clinic of Child Psychiatry, University of Oulu, Oulu, Finland irma.moilanen@oulu.fi

Objectives. To study the inter-twin relationships, development and mental health in a longitudinal study.

Methods. All twins born in the University Central Hospital of Oulu in the years 1965-72 were followed from pregnancy up to 23-30 years'age by gathering data from hospital records, children's health care centres, by questionnaires filled in by the parents and the twins themselves and by personal interviews to a sample of twins.

Results. Mutual inter-twin dependence decreased from $68 \%$ before school age to $50 \%$ at school age and $10 \%$ after school, while one-sided co-twin dependence of either twin changed from $5 \%$ to $11 \%$ and $3 \%$, respectively. The degree of dominance-submissiveness relationship between twins decreased so, that $82 \%, 85 \%$ and $95 \%$ of the twins reported of having felt equal before school age, at school and later, respectively. While studying the relationships to parents, the corresponding figures of being closer to the mother were $17 \%, 22 \%$ and $25 \%$ and closer to the father $7 \%, 8 \%$ and $3 \%$, respectively. All these three special features: co-twin dependence, dominance-submissivenes and parental preferences had some connections with psychosomatic symptoms and depressiveness.

Conclusions. Inter-twin relationships should be taken in account when studying mental health on twins.

\section{S THE GENETICS OF DIZYGOTIC TWINNING}

\section{Grant W. Montgomery, David L. Duffy, Dorret I. Boomsma} and Nicholas G. Martin

Correspondence address: Genetic Epidemiology Unit, Queensland Institute of Medical Research and Joint Genetics Program, The University of Queensland, Brisbane, Queensland, Australia

Natural pregnancy in women leading to dizygotic twins clusters within individuals and in families and is under genetic control. Gene discovery, gene knockout and positional cloning in animal models have recently identified a new pathway controlling multiple ovulation and twinning frequency. Growth factors expressed in the oocyte have key roles in fertility and infertility. Mutations in the growth differentiating factor BMP15 and the receptor BMPR1B increase ovulation rate and the frequency of twins in sheep. One important question is whether natural variation in human twinning is controlled by these emerging mechanisms or through other pathways. The analysis of markers spanning the critical region of chromosome $4 \mathrm{q}$ containing BMPR1B did not show linkage to this region. Several recent reports directly implicate three genes, follicle stimulating hormone receptor (FSHR), peroxisome proliferator activated receptor (PPARG) and methylenetetrahydr ofolate reductase (MTHFR) in the etiology of twinning. The results of linkage analysis in our families do not support roles for FSHR or PPARG in the familial aggregation of twinning within the population we have studied, which is largely of British and Irish extraction. We are continuing our search for the genes responsible for natural twinning with additional candidate studies to provide a greater understanding of female fertility and infertility.

\section{F ISSUES IN THE BIOMETRICAL GENETIC ANALYSIS OF MRI BRAIN VOLUME DATA}

Michael C. Neale, Elizabeth A. Molloy, Jonathan D. Blumenthal,Liv Clasen \& Jay N. Giedd

Correspondence address: Department of Psychiatry, Box 980126, Richmond VA 23298-0126

The analysis of MRI twin data presents some novel challenges for quantitative genetic analysis, such that the method used may depend on the objective of the study. Analysis of raw data without correction for covariates provides an estimate of the relative proportions of additive genetic, shared and specific environment variance that includes effects such as height and weight, which are known to have substantial heritabilities, and also of age, which behaves like common environment when the members of twin pair are assessed at the same age. Subtraction of the effects of age is readily justified, but it is less clear whether height weight 
or sex should be removed from the analysis, particularly when the relationship between brain volumes and cognitive abilities is the focus of the study. These issues are illustrated with some preliminary analyses of data being collected on twin pairs aged between 7 and 16 years at the National Institute of Mental Health.

\section{P NEONATAL OUTCOMES OF PRETERM TWINS}

Dorota Nowakowska, Jan Wilczynski, Bogumil Jaczewski,Jaroslaw Oleszczuk, Grzegorz Krasomski, Przemyslaw Oszukowski

Correspondence address: Institute Polish Mother's Memorial Hospital, Rzgowska 281/289 Str.,93-338Lodz,Poland,ndorota@hotmail.com

Purpose.

The objective of the study was to compare neonatal outcomes of preterm twins and singletons.

\section{Material and Methods.}

Obstetrics and neonatal data were collected retrospectively and included 38062 deliveries between 1989 and 1998. Out of 538 sets of twins, $312(58.0 \%)$ delivered before 37 weeks of gestation; compared with singletons preterm birth rate of $11.7 \%$ Preterm birth was defined as delivery before 37 weeks of gestation. The exclusion criteria were a gestation age less than 24 weeks or a birth weight less than $500 \mathrm{~g}$. Results.

\begin{tabular}{lccc} 
Morbidity & $\begin{array}{c}\text { Singletons } \% \\
(N=4323)\end{array}$ & $\begin{array}{c}\text { Twins } \% \\
(N=601)\end{array}$ & $\begin{array}{c}\text { Relative risk } \\
(95 \% \mathrm{CI})\end{array}$ \\
\hline Sepsis & $20,7(894)$ & $38,1(229)$ & $1,84(1,64-2,07)$ \\
IVH III\&IV & $8,1(352)$ & $17,8(107)$ & $2,19(1,79-2,67)$ \\
RDS & $24,6(1062)$ & $37,4(225)$ & $1,52(1,36-1,71)$ \\
NEC & $6,7(291)$ & $18,3(110)$ & $2,72(2,22-3,33)$ \\
\hline
\end{tabular}

Conclusions. Compared with singletons, twins delivered before 37 weeks of gestation contributed significantly higher rates of sepsis, respiratory distress syndrome (RDS), intraventricular haemorrhage (IVH) and necrotizing enterocolitis (NEC)

\section{P NO RISK OF COAGULOPATHY AFTER SINGLE INTRAUTERINE DEATH IN TWIN PREGNANCY}

Dorota Nowakowska, Jan Wilczynski, Bogumil Jaczewski, Lech Podciechowski, Grzegorz Krasomski, Przemyslaw Oszukowski

Correspondence address: Institute Polish Mother's Memorial Hospital, Rzgowska 281/289 Str.,93-338Lodz,Poland, ndorota@hotmail.com

Purpose. The aim of our the study was to compare the frequency of the consumption coagulopathy in monochorionic (MCG) and dichorionic (DCG) twin gestations with single intrauterine death (SIUD).

Material and Methods. During a 10 year period (1989-1998) we identified 26 out of 538 twin gestations involving SIUD. Monochorionic placenta was found in $16(61.5 \%)$ cases.The coagulation tests performed 2-3 times per week after SIUD diagnosis included: platelet count (PLT), fibrinogen concentration in plasma (FBG), prothrombin time (PT), thrombin time (TT) and kaolin cephalin time (KCT). The anticoagulants were not administered in pregnancies with SIUD.

Results. The results of hemostatic tests were the indication for consumption coagulopathy in any case. PLT count did not significantly differ in MCG and in DCG: 240.12 G/l (S.D.58.03) vs. 240.60 G/l (S.D.58.48). There were any significant differences observed between FBG values between MCG and DCG with SIUD: $3.02 \mathrm{~g} / 1$ (S.D.0.7) vs. 3.15 g/l (S.D.0.89). The results of prothrombin time, thrombin time and kaolin-cephalin time did not significantly differ in MCG and DCG and were within normal range of values. There was no difference between monochorionic and dichorionic pregnancies in PLT $(p=0.32)$; FBG $(p=0.60) ; \operatorname{KCT}(p=0.67) ; \mathrm{PT}(p=0.69) ; \mathrm{TT}(p=0.13)$.

Conclusion. The results of the hemostatic tests did not significantly differ in MCG and DCG with SIUD and they were in any case indicative for consumption coagulopathy.

\section{P EXAMINATION OF INTRAPARTUM CARDIOTOCOGRAMS AND SLEEP-AWAKE PERIODS IN SINGLETON AND TWIN FOETUSES DURING THE SECOND STAGE OF LABOUR}

Ildikó Nyirati, Judit Bartkó, György Bártfai

University of Szeged, Dept. Ob/Gyn, Hungary-6725, Szeged, Semmelweis u.L.

Introduction. Intrapartum cardiotocography provides an early diagnosis of foetal jeopardy. A decreased foetal heart rate variability and bradycardia are good indicators of a transient impaired placental reserve capacity. In cases with a physiological reserve capacity of the placenta, uterine contractions do not cause any pathological changes in the foetal heart rate

Aim. To examine the foetal sleep-awake periods during the last three hours of the second stage of labour and to compare the cardiotocograms with the perinatal outcome.

Materials and Methods. The intrapartum cardiotocograms of 300 singleton and 42 twin pregnancies were subjected to a retrospective analysis and the recordings and foetal outcome were compared.

Results. During the last three hours of the second stage of labour, there were $4.3,4.8$ and 4.5 periodical changes in sleep-awake states for singleton, twin "A" and twin "B" foetuses with, on average two activity periods (F4) in all groups. The mean umbilical venous blood $\mathrm{pH}$ was 7.27, 7.28 and 7.25 , retrospectively. In singleton pregnancies, depending on the $\mathrm{pH}$ level $(<7.20,7.20-7.25$ and $>7.25)$, significant differences were found in the periodical changes, the number of F4 periods and the number of decelerations. Similar trends were registered in twin foetuses.

Conclusion. A decreased number $(<4)$ of sleep-awake periods and/or activity cycles $(<2)$ can alert the physician to foetal hypoxia. It appears very important to register the foetal sleep-awake periods and foetal movements in addition to the foetal heart rate during labour.

\section{F EARLY BREAST-FEEDING OF MULTIPLE NEWBORNS}

Ildikó Nyirati, Hajnalka Orvos, Zoltán Kozinszky, György Bártfai University of Szeged, Dept. Ob/Gyn, Hungary-6725, Szeged, Semmelweis u.

Introduction. In recent decades, there has been an increasing tendency in the incidence of multiple pregnancies in consequence of the use of assisted reproductive techniques. In the early postpartum period, the feeding of the newborns is an enormous challenge for the mothers of multiples.

Aim. To analyse the characteristics of breast-feeding in multiple pregnancies (two or more newborns).

Materials and Methods. Between 1st January, 1994 and 31st December, 1998, all data on multiple pregnancies involving at least 24 weeks of gestation were collected at the Department of Obstetrics and Gynecology, Albert Szent-Györgyi Medical University, Szeged, Hungary.

Results: Three groups were created on the basis of the birth-weight: 1. Each newborn weighed between 2000 and $2500 \mathrm{~g} ; 2$. only the largest newborn weighed at least $2500 \mathrm{~g}$; and 3. each newborn weighed at least 2500 g. Early breast-feeding was carried out in $29.4 \%$ of group 1., in $89.1 \%$ of group 2 . and in $95.0 \%$ of group 3. More than $80 \%$ of the early breast-fed newborns were exclusively breast-fed at discharge. In three triplet pregnancies, all newborns were breast-fed early in the postpartum period and all of these mothers had an appropriate milk yield for their triplets.

Conclusion. This study confirmed that, under optimum circumstances, the breast is capable of producing sufficient milk to nourish two or even three infants. As the milk production is a very important factor in the successful nursing of multiples, every effort should be made to maximize the milk supply in the early postpartum period.

\section{$181 \mathrm{P}$}

\section{MONOAMNIOTIC TWIN PREGNANCY - FOUR CASE REPORTS AND REVIEW OF THE LITERATURE}

Jaroslaw J. Oleszczuk, Michal Krekora, Dorota Nowakowska, Lech

Podciechowski, Ewa Czichos, Agnieszka K. Oleszczuk, Bozena LeszczynskaGorzelak, Jan Wilczynski, Louis G. Keith

Correspondence address: Department of Maternal-Fetal Medicine, Research Institute - Polish Mother's Memorial Hospital, ul. Rzgowska 281/289, 93-335 Lodz, Poland jarek@obgyn.net

Monoamniotic twin pregnancies are rare. They comprise only about $5 \%$ of all monozygotic twin pregnancies (about 1 in 10-20 thousand deliveries). The most serious complication which leads to intrauterine death in $50 \%$ of MA pregnancies is cord entanglement. Early diagnosis is feasible by color Doppler and pulsed Doppler velocimetry. We report of two cases of monoamniotic twin pregnancies hospitalized in our respective institutions. In both instances, diagnosis of entanglement prompted more scrutinous surveillance and led to an elective cesarean delivery at 32 weeks' gestation. We also report of two other cases of monoamniotic twin pregnancies in which fetal demise was diagnosed at midtrimester the cause of which was cord entanglement. Based upon these cases,we provide a review of the literature concerning the topic of monoamniotic pregnancy and attempt to provide a diagnostic and therapeutic protocol for managing MA pregnancies which aims at optimizing perinatal outcomes. 
182P EVALUATION OF VENOUS DOPPLER FOR ASSESSING FETAL WELL-BEING IN TWIN PREGNANCY.

Jaroslaw J. Oleszczuk,Agnieszka K. Oleszczuk, Bozena Leszczynska-Gorzelak, Jan Wilczynski, Louis G. Keith

Correspondence address: Department of Obstetrics and Perinatology, Medical University of Lublin, ul. Jaczewskiego 8, 20-954 Lublin, Poland jarek@obynn.net

Even though fetal Doppler has become a widely accepted technique of evaluating fetal well-being in singleton and twin pregnancies, data on the application of fetal venous Doppler in twin pregnancies is scant.

Aim. To evaluate fetal well being in twin pregnancies using color Doppler and pulsed Doppler velocimetry of ductus venosus, inferior vena cava, pulmonary vein, umbilical vein, as well as umbilical artery, middle cerebral artery, ductus arteriosus and AV valves.

Methods. All twin pregnancies hospitalized at two Polish reference hospitals between August 2000 and May 2001 were included in the study. Color Doppler and pulsed Doppler velocimetry studies were performed in all fetuses. The standard flow parameters were calculated for each blood vessel tested. Antepartum conclusions were confronted with perinatal results.

Results and Conclusions. Fetal venous Doppler had a high sensitivity for detecting fetuses at risk for perinatal complications in the tested cohort. Its accuracy is often questioned but methodology can reliably be applied by experienced ultrasonographists. Venous Doppler studies permit a more accurate assessment of fetal oxygenation and appropriate decisions can be made regarding perinatal management.

\section{F PROJECTIONS OF POPULATION-BASED TRIPLET BIRTH RATIOS THROUGHOUT THE NEXT DECADE - IS THE EPIDEMIC UNCONTROLLABLE?}

Jaroslaw J. Oleszczuk,Agnieszka K. Oleszczuk, Bozena Leszczynska-Gorzelak, Jan Wilczynski, Louis G. Keith

Correspondence address: Department of Maternal-Fetal Medicine, Research Institute Polish Mother's Memorial Hospital, ul. Rzgowska 281/289, 93-335 Lodz, Poland jarek@obgyn.net

Triplet pregnancies are high risk for both the mother and the fetus. Their main complication is preterm delivery of a low or very low birthweight neonate who always requires prolonged stays at the NICU. Long term consequences are even more dramatic and include a three times higher risk of cerebral palsy than in singleton pregnancies. In the past 20 years, the triplet birth ratio $(\mathrm{TBR}=$ triplet births per 100,000 live births) has been quite steady. Only recently, in the late 1980 s and 1990s, the TBR has increased dramatically in almost all developed countries. This phenomenon is thought to be a consequence of better access to infertility treatment (both in-vitro fertilization and ovulation induction) which carries an inherent risk of multiple pregnancy. Population-based TBRs from various countries have been analyzed and a projection made throughout the next decade. Results are startling - if TBR increases were to continue at the rate they have until now, triplets would comprise a third of all live births in some countries. The consequences of this potentially uncontrollable rise in triplet births for both health systems as well as parents are difficult to envision. Current infertility treatment practices need revision in order to control this potentially devastating epidemic.

\section{P EVALUATION OF VARIOUS FETAL GROWTH CURVES FOR THE ASSESSMENT OF IUGR IN TWINS}

Jaroslaw J. Oleszczuk, Agnieszka K. Oleszczuk, Bogumil P. Siekierski, Bozena Leszczynska-Gorzelak, Jan Wilczynski, Louis G. Keith

Correspondence address: Department of Obstetrics and Perinatology, Medical University

Correspondence address: Department of Obstetrics and Perinatin, ul. Jaczewskiego 8, 20-954 Lublin jarek@obgyn.net
of Lublin

It is widely accepted that the pattern of fetal growth for twins is the same as for singleton up to 30 weeks' gestation. However, intrauterine growth of twins recedes after this yielding a hypotrophic fetus. Recent evidence suggests that growth references for twins should be the same as for singletons in order to appropriately evaluate fetal growth.

Aim. To evaluate various growth curves for the evaluation of fetal growth aberrations in twin gestations.

Methods. Growth reference curves for twins were constructed using ultrasonically estimated fetal weights of all twin pregnancies with no detected congenital anomalies delivered between 1987 and 2000 in two reference hospitals in Poland. Fetal growth was then prospectively evaluated in twin pregnancies hospitalized between 2000 and 2001 Assessment was achieved using 3 growth curves - the constructed reference curve for Polish twins, the standard singleton reference curve and the reference curve for twins in the United States. Antepartum conclusions were confronted with perinatal results.
Results. The constructed fetal growth reference curve for twins provides a new, population-specific tool of growth assessment in twin pregnancies. All three curves used gave high positive predictive values for the outcome measures tested.

CONGENITAL HYPOTHIROIDISM IN TWINS (ITALY, 1989-1999)

Antonella Olivieri, Emanuela Medda, Cristina Fazzini, Mariella Sorcini,

Rodolfo Cotichini, M.Antonia Stazi

Correspondence address: Istituto Superiore di Sanità, Rome, ITALY

Neonatal screening program for congenital hypothyroidism $(\mathrm{CH})$ started in Italy in 1977 and then progressively developed covering all neonatal population. The National Register of infants with $\mathrm{CH}(\mathrm{NRCH})$ was established in Italy in 1987. An ongoing case-control study, in which 168 incident $\mathrm{CH}$ cases from the $\mathrm{NRCH}$ and 666 matched controls have been enrolled, estimates that twins have an $\mathrm{OR}=12$ (95\% CI: $4.1-36)$ to be $\mathrm{CH}$. However, no extensive studies have been performed to evaluate the occurrence of $\mathrm{CH}$ in twin pregnancies. The aims of the present study were: 1) to estimate the prevalence of twins in the Italian population of infants with $\mathrm{CH}$ recorded in the $\mathrm{NRCH}$; 2) to compare the frequency of twins in the $\mathrm{CH}$ population with that observed in the general population; 3) to obtain an unbiased estimate of MZ and DZ twin concordance rates for $\mathrm{CH}$; 4) to compare family history, neonatal features and clinical findings between singleton and twin infants with $\mathrm{CH}$. Between 1989 and 1999, $1872 \mathrm{CH}$ infants were identified among 5,863,700 live births screened in Italy in this period $(\mathrm{CH}$ incidence $=1: 3132)$. Among the 1872 $\mathrm{CH}$ babies, 70 were twins, with a proportion of multiple pregnancies among all the $\mathrm{CH}$ pregnancies of $65 / 1867$ i.e. $3.5 \%$ versus a proportion of $1.1 \%$ of multiple pregnancies estimated (in all pregnancies) in Italy in the same period. Among the $70 \mathrm{CH}$ twins only three concordant pairs (all male-male pairs) and one concordant triplet (two girls and one boy) were observed. All $\mathrm{CH}$ twins are going to be traced in the centres in charge for the follow-up in order to have information on zygosity.

\section{S} CONJOINED TWINS - WHETHER TO SEPARATE

James A. O'Neill, Jr., M.D.,

Correspondence address: Vanderbilt University Medical Center, D-4316 MCN, Nashville, Tennessee 37232

Current accepted treatment of conjoined twins involves separation whenever reasonably possible. In some instances, the decision and the surgery are straightforward, but the decision can be problematic, particularly when survival of only one twin is possible. The author has been involved in decision-making and operative planning in nearly 50 cases and in separation surgery in 24 instances. An attempt has been made to make a realistic assessment of the chances of life and of a quality life. It is important to realize that conjoined twins often have associated congenital anomalies. In general, the decision to separate omphalo-, ischio-, pygo-, and heteropagus twins is straightforward and the outlook is good with few exceptions. Many of these separated infants require subsequent operations but, in general, the quality of life is good. Most craniopagus twins cannot be separated because of the poor outlook for both of the twins. The majority of thoracopagus twins who share hearts cannot be separated but sometimes one twin can survive. Issues related to the decision which will be discussed include psychological adjustment of separated vs. non-separated twins, comfort and dependency in nonseparated twins, and the right to life, bodily integrity, human dignity and privacy. The moral, ethical, sociological and legal aspects of problematic cases will be discussed. Long-term follow-up data on separated conjoined twins supports a reasonable approach to separation of conjoined twins whenever feasible as the preferred approach.

187P

\section{PHYSICAL GROWTH, MOTOR DEVELOPMENT AND LANGUAGE DEVELOPMENT STANDARDS OF JAPANESE TWIN INFANCY}

\section{S. Ooki and A. Asaka}

Correspondence address: Department of Public Health Toho University School of Medicine 21-16 Omori-Nishi 5-Chome Otaku Tokyo 143-8540 Japan.syuoo@med.toho-u.ac.jp

Few basic data is available concerning the general growth and development of twins in Japan, partly because there exists no registry system for multiple-birth. The present study aimed at presenting twins' physical growth, motor development and language develop ment standards in infancy. Subjects were 894 pairs of normally developed Japanese twins born from 1968 to 1988 , consisting of $670 \mathrm{MZ}$ and $224 \mathrm{DZ}$ twin pairs. The following results were obtained. (1) Size deficit at birth was appropriately recovered over the first 6 years especially for weight. (2) The difference between singletons and twins were small as to motor development, except for the beginning month of standing. (3) As to language development (have one or two words), twins' development gradually delayed after one year of age. (4) The similarity between MZ and DZ pairs was almost the same at birth. However, MZ pairs became 
rapidly more similar with age, whereas DZ pairs became more and more dissimilar. This tendency was very clearly seen through first one year, suggesting that genetic factors play a substantial role during this life stage. Further analysis will be made with another one hundred pairs data.

\section{F PRIMARY OSTEOARTHRITIS OF THE HIP} IN MONOZYGOTIC AND DIZYGOTIC MALE TWINS

William F. Page, Franklin T. Hoaglund, Lynne S. Steinbach,Andrew C. Heath. Correspondence address: Institute of Medicine, 2101 Constitution Avenue, N.W., Washington, DC 20418, USA

This study was undertaken to determine the genetic inheritance of primary osteoarthritis of the hip by comparing coincidence of disease in identical and fraternal twin pairs. 6,419 male veteran twins born between 1917 and 1927 were identified from the NAS-NRC Twin Registry, contacted by telephone, and asked whether they had a total hip replacement for arthritis rather than fracture. Twin pairs in which one twin reported a total hip replacement were contacted to complete a written questionnaire and to give permission for the recovery of medical information and appropriate $\mathrm{x}$-rays. Cotwins not having a total hip replacement were invited to have a pelvic $\mathrm{x}$-ray. X-rays were read separately and conjointly by an orthopedist and musculo-skeletal radiologist without knowledge of zygosity or cotwin. Data were analyzed using a 2-stage Mx model for the telephone screening and x-ray data. 2,361 twins pairs provided telephone data in the survey, and x-ray diagnosis data was available for 61 probands identified by telephone survey. In the best fitting model, additive genetics accounted for 53\% in the liability for self-reported hip replacement surgery (95\% CI 30-72\%) and unique environment for the remaining $47 \%$. Additive genetics accounted for $61 \%$ of the variance in liability for $\mathrm{x}$-ray determined primary osteoarthritis $(95 \% \mathrm{CI} 18-86 \%)$ with unique environment accounting for the remaining 39\%. Genetics had a significant influence on the development of primary osteoarthritis of the hip in this group of male veteran twins aged 70-80 years. Further work is indicated to isolate the genes responsible.

\section{P NATIONAL BIRTHWEIGHT STANDARDS BY GESTATIONAL AGE FOR TWINS AND MULTIPLES (ITALY 1981-1995)}

Valeria Patriarca, Sonia Brescianini,Rodolfo Cotichini, Cristina D'lppolito, M.Antonia Stazi

Correspondence address: Istituto Superiore di Sanità, Rome, ITALY

The aim of this study was to compare birth-weight by gestational age between single, twin, and multiple live-births.

Historical population-based data has been used. All new-borns in Italy from 1981 to 1995 are considered and grouped into three cohorts: 81-85, 86-90, 91-95 (data source: National Institute of Statistics). The abstract concerns the 1991-1995 cohort. There were 2,751,803 births: $97.79 \%$ singletons, $2.09 \%$ twins, and $0.12 \%$ multiple births. For singletons, the proportion of males was $51.6 \%$, stillbirths were $0.45 \%$, and birth defects were $0.43 \%$. The percentages were $50.7 \%, 1.63 \%, 0.55 \%$, for twin pairs, and $51.5 \%, 2.54 \%, 0.56 \%$ for multiple births, respectively. The cohorts have been defined according to the inclusion criteria described in the literature: stillbirths, birth defects, outliers, and potential errors were excluded.

A preliminary descriptive analysis of some characteristics of the study sample, for the period 1991-1995 is given.

\begin{tabular}{lcccc} 
& $\begin{array}{c}\text { maternal age } \\
\text { (year) }\end{array}$ & caesarean & $\begin{array}{c}\text { weight } \\
\text { (grams) }\end{array}$ & $\begin{array}{c}\text { weight } \\
\text { percentiles }\end{array}$ \\
\hline singleton & mean $S D$ & $\%$ & mean $S D$ & $10^{\circ}-90^{\circ}$ \\
twin & $28.7 \pm 5.0$ & 23.36 & $3292 \pm 495$ & $2726-3884$ \\
multiple & $29.5 \pm 4.9$ & 57.56 & $2413 \pm 553$ & $1702-3042$ \\
\hline
\end{tabular}

National birth-weight standards by gestational age for twins and multiples compared to singletons are under development and will be presented. The time trend (1981-1995) will be also evaluated.

\section{S WHAT HAVE TWIN STUDIES TAUGHT US ABOUT AGING?}

\section{Nancy L. Pedersen}

Correspondence address: Department of Medical Epidemiology, Karolinska Institutet, Bo 281, SE 17177 Stockholm, Sweden

Previous lifespan developmental theories predicted that as people grow older, they would become more different, primarily due to an increase in environmental experiences and exposures. Results from twin and adoption studies in the first half of the lifespan suggested that genetic effects are amplified during development, and that genetic influences contribute to both stability and change. Considerable longitudinal twin data have become available since the mid 1980's, covering most phases of the lifespan (although data on midlife are sparse). Advances in analytic techniques, including simplex and latent growth curve models, have enabled us to address a number of new issues concerning variability in aging. For example, with these tools we are now able to evaluate the role of morbidity and impending mortality on the timing and rate of "terminal decline". Using longitudinal data on personality, cognitive abilities, and health outcomes from the Swedish Adoption/Twin Study of Aging ("young-old") and the OCTO-Twin study ("oldest old"), I will review the empirical evidence for and against a number of "developmental principles" in the last half of the lifespan.

\section{S CHRONIC FATIGUE SYNDROME IN THE SWEDISH TWIN REGISTRY}

Nancy L. Pedersen and Patrick Sullivan

Correspondence address: Department of Medical Epidemiology, Karolinska Institutet, Box 281, SE 17177 Stockholm, Sweden

Chronic fatigue syndrome (CFS) is a complex disorder of unknown etiology. Unlike many complex traits in medicine, there are very few data from family, adoption, or twin studies that address its fundamental etiology. Thus, we will present data from a population-based classical twin study of CFS-like illness. These data are from a recent computer assisted telephone screening of 11,928 participants in the Swedish Twin Registry aged 55-65 years (74\% response rate). Preliminary results suggest that CFS-like illness is substantially heritable in this sample.

\section{F INTER-TWIN RELATIONS} AND INTER-TWIN DEPENDENCE

Varpu Penninkilampi,Irma Moilanen, Jaakko Kaprio

Correspondence address: University of Oulu, Clinic of Child Psychiatry, P.O. Box 22, FIN-90220 Oulu, Finland.Varpu.Penninkilampi@oulu.fi

Research of twins has traditionally been oriented towards the study of genetics. Most studies are concentrated on the roles of heredity and environment in the development of many physical, somatic, cognitive, psychiatry and personality traits. In many studies (shared) environment include such factors as influences of siblings, common peers and parents. However the twinship itself constitutes a special situation, which could have effects on development, behavior and health of an individual. Though twins can support each other in many ways, twinship can also have negative effects on individual development, if twins have identified strongly with each other. The objective of this study was to investigate interpersonal relationship in adolescent twins. The main areas of interest were twins' and their parents (mothers) perceptions of inter-twin dependence, reciprocal roles and dominancesubmissiveness among twins. These aspects were analyzed with special regard for zygosity, sex and pubertal development. This study is part of an ongoing nation-wide, longitudinal study of five consecutive birth cohorts of adolescent Finnish twins. Data consist of FinnTwin16 $(N=5747$ twin individuals, 2711 pairs of known zygosity). The questionnaires were administered sequentially to all twins, born 1975-79, as they reached age 16 . Additional questionnaires were sent to the parents (Rose et al., 1999).

The future aim is to study the consequences of inter-twin relationships and dependence on development, behavior and health of twin children and adolescents.

\section{S SURVIVAL AND NEUROLOGICAL OUTCOME IN TWINS: RELATION TO ANTENATAL GROWTH}

\section{Beverly Petterson and Eve Blair}

Correspondence address: Disability Services Commission, 146-160 Colin St West Perth 6005 WA Australia

All twins born in Western Australia 1980-1992 $(N=7200)$ were ascertained from the Maternal and Child Health Research Database, which registers all births in the state ( $>20$ weeks or $>400 \mathrm{~g}$ birthweight) and includes a comprehensive collection of perinatal variables. These data can be computer linked to the statutory Registry of Deaths and the Cerebral Palsy, Birth Defects and Intellectual Disability databases. Ascertainment for cerebral palsy (CP) and birth defects is considered to be virtually complete and is estimated to be $80 \%$ for intellectual disability (ID).

\begin{tabular}{|c|c|c|c|c|c|c|c|}
\hline & \multicolumn{3}{|c|}{ Deaths } & \multirow[t]{2}{*}{ CP only } & \multirow[t]{2}{*}{$C P+I D$} & \multirow[t]{2}{*}{ ID only } & \multirow{2}{*}{$\begin{array}{l}\text { Other } \\
\text { Survivors }\end{array}$} \\
\hline & & Ante-natal & $\begin{array}{l}\text { Birth } \\
\text { to } 1 \mathrm{yr}\end{array}$ & & & & \\
\hline $\mathrm{N}$ & & 153 & $342 \#$ & 37 & 25 & 42 & 6601 \\
\hline$\%$ births & & 2.13 & 4.75 & 0.51 & 0.35 & 0.58 & 91.7 \\
\hline \multirow[t]{2}{*}{$\%$ major } & any & $10.8^{*}$ & 16.9 & 2.7 & 28.0 & 45.2 & 5.0 \\
\hline & cns & $2.5^{*}$ & 4.4 & 0.0 & 16.0 & 0.0 & 0.3 \\
\hline Growth ** & & - & 83.9 & 95.2 & 88.4 & 77.9 & 85.9 \\
\hline
\end{tabular}

* 33 with missing data for BD excluded. ${ }^{* *}$ Mean proportion of birthweight expected for gestation of delivery, gender and maternal height. \#excludes 3 cases diagnosed before death by the age of 1 year. 
ID was associated with both major birth defects and restricted intrauterine growth, whereas CP in the absence of ID was associated with neither. Indeed twins with $\mathrm{CP}$ tended to be better grown than normal surviving twins.

\section{P ERRORS IN BIRTH REGISTRATION AND CODING OF MULTIPLE BIRTHS IN NATIONAL STATISTICS}

\section{Peter O.D. Pharoah}

Correspondence address: Department of Public Health, University of Liverpool, Liverpool $L 693 G B$

Aim. To validate the coding and registration of gender and number of births in multiple pregnancies in national statistics.

Methods. Examination of birth, fetal and infant death registrations of all multiple maternities in England and Wales 1993-8.

Results. There were 51,792 twin, 1627 triplet and 51 higher order maternities. Of the 1926 fetal deaths among multiple maternities, 58 were registered as 'indeterminate' sex but were coded 'male' in 56 and 'female' in 2 instances. A fetus papyraceous was the certified cause of death in 55 instances; 19 were registered 'male', 19 'female' but only 17 were registered as 'indeterminate' sex. In 13 maternities only twins were registered but mention was made on the death certificate that 9 were from triplet and 4 from quadruplet pregnancies.

Conclusion. It cannot be assumed that multiple births of different registered sex are always from dizygotic conceptions. Because surviving infants from monozygotic are at greatly increased risk of death and serious morbidity compared with dizygotic conceptions, incorrect assignation and coding of sex and of the number of fetuses has important implications for parental counselling.

\section{$195 \mathrm{~F}$}

\section{NEONATAL MORTALITY IN LIKE (LS) AND UNLIKE (US) SEX TWINS AND CEREBRAL PALSY (CP)} IN THE CO-TWIN SURVIVOR OF AN INFANT DEATH

\section{Peter O.D. Pharoah}

Correspondence address: Department of Public Health, University of Liverpool, Liverpoo $L 693 G B$

Aim. To determine the risk of CP in a twin whose co-twin died in infancy. Methods: Analysis of birth and death registrations for LS and US twins for England \& Wales 1993-5 where both were live born. A questionnaire was sent to the general practitioner of all surviving twins to determine if the child had any disability.

Results. The neonatal mortality was 25.4 in LS and 18.0 per 1000 live births in US twins (mortality rate difference $7.4 ; 95 \%$ CI 4.7 to 10.1 ; $p<0.001)$. This difference is attributable to LS twins being of lower birthweight. Among survivors of birthweight $<1000 \mathrm{~g}$, there was no significant difference in CP rates in LS and US twins (213 and 220 per 1000 respectively). In survivors of birthweight $1000-1999 \mathrm{~g}$ the CP prevalence in LS twins was 162 and in US twins was 21 per 1000 . The difference was highly significant; $p=0.01$.

Conclusion. There are two components to the aetiology of CP in twins who are both live born. Immaturity per se predisposes to cerebral impairment. In addition, LS twins may sustain cerebral impairment that is in excess of that due to immaturity.

\section{P}

\section{SUDDEN INFANT DEATH SYNDROME (SIDS)} IN TWINS

Peter OD Pharoah, D Anand, Mary Jane Platt

Department of Public Health, University of Liverpool, Liverpool L69 $3 G B$

Aims. To compare the incidence of SIDS in like sex (LS) and unlike sex (US) twins.

Methods: All twin births in England \& Wales 1993-8 comprise the denominator cohort. The death certificates of those who died were provided by the Office for National Statistics. All SIDS by birthweight group, age at death and whether they were LS or US comprised the numerator.

Results. There were 68,124 LS livebirths with 78 SIDS, a SIDS mortality rate of 1.14 per 1000 . Among 33,734 US livebirths there were 42 SIDS, a SIDS mortality of 1.25 per 1000 . The LS-US difference in SIDS mortality was not significant. SIDS from LS twins were of significantly lower birthweight than those of US twins. There was no significant difference in the age at death.

Conclusions. Zygosity does not appear to be of importance in twin SIDS deaths. The difference in birthweight distribution is probably because LS twins are of lower birthweight than US twins.
Peter OD Pharoah,D Anand, Mary Jane Platt, Leslet Briscoe. Correspondence address: Department of Public Health, University of Liverpool, Liverpool L69 $3 G B$.

Aims. To determine the prevalence of a multiple pregnancy at first ultrasound in pregnancy and the outcome at time of delivery.

Methods. A cohort of all pregnancies with 2 or more sacs seen at first ultrasound in pregnancy (usually at 10-12 weeks gestation) at the Liverpool Women's Hospital in 1999/2001 comprised the study sample and were followed through to delivery.

Results. There was a total 10,000 maternities of which 258 (26 per 1000 maternities showed 2 sacs and 10 showed 3 sacs $(1.0$ per 1000 maternities) at the first ultrasound examination.O f the 258 twin sacs, in 18 $(7.0 \%)$ both aborted and in $70(27.1 \%)$ one vanished so that at delivery there were 170 twin pairs (17.0 per 1000 maternities) and 70 singletons (7.0 per 1000 maternities).Of the 10 triplet sacs, in 2 all three fetuses aborted and in 2 others, 1 fetus vanished and twins were delivered. Six delivered triplets.

Conclusions. If early loss of one fetus in a multiple pregnancy has adverse consequences such as cerebral impairment leading to cerebral palsy or learning disability, the high frequency of a vanishing fetus in a multiple pregnancy may be responsible for a considerable proportion of cerebrally impaired children.

\section{$198 \mathrm{~F}$}

\section{THE EFFECTS OF ASSISTED REPRODUCTION IN THE TRENDS AND ZYGOSITY OF MULTIPLE BIRTHS: ENGLAND AND WALES 1974-99}

Peter O.D. Pharoah, Mary Jane Platt, Amelia Marshall.

Correspondence address: Department of Public Health, University of Liverpool, Liverpool $L 693 G B$

Aim. To examine trends in the prevalence of multiple pregnancy by zygosity and to estimate the contribution assisted reproduction to monozygotic twinning.

Methods. National data on multiple births, 1974-99 were used and subdivided into 4 periods: $1974-80$ (pre-assisted reproduction); 1982-88; 1989-91(pre-redefinition of stillbirth) and 1993-99(post-redefinition of stillbirth). Weinberg's rule was applied to twin data to estimate the proportions that were mono- and dizygous. The assumption is made that the increase in both MZ and DZ twins is attributable to assisted reproduction.

Results. Compared with the period before assisted reproduction, the most recent period shows an increase in twin maternities of 3.81 per 1000 comprised of $3.22(95 \%$ CI $3.10-3.33 ; p<0.0001) \mathrm{DZ}$ and $0.60(95 \%$ CI $0.51-0.68 ; p<0.0001) \mathrm{MZ}$ twins per 1000 maternities. It is estimated that $15.7 \%$ of assisted reproduction maternities are associated with $\mathrm{MZ}$ division.

Conclusion. As MZ compared to DZ conceptions are at greatly increased risk of fetal and infant death, the effects of assisted reproduction need consideration when auditing hospital fetal and perinatal mortality rates.

\section{P VERBAL FLUENCY IN TWINS WITH SCHIZOPHRENIA: AN fMRI STUDY}

Marco Picchioni,Vivienne Curtis, Cindy Fu, Xavier Chitnis, John Suckling, Robin Murray, Philip McGuire

Correspondence address: Division of Psychological Medicine, Institute of Psychiatry, London, UK.

Abnormal prefrontal and temporal lobe activation during word generation has been widely reported in schizophrenia. We investigated genetic and non-genetic influences on these abnormalities by studying MZ twin pairs with functional MRI. Twins discordant and concordant for schizophrenia and healthy twins were compared using a paced verbal fluency paradigm. Word generation cued by letters was contrasted with repetition of the word 'Rest' in an AB design. Images were acquired at 1.5T using a compressed acquisition sequence to allow overt response in the absence of scanner noise. Activation maps in Talairach space were generated using established nonparametric methods. Within discordant twins, schizophrenia was associated with attenuated activation in the right inferior frontal and temporal cortex and the medial prefrontal cortex. Compared to control twins, healthy discordant twins showed reduced activation in the left inferior frontal and temporal cortex and the cingulate gyrus. These initial results suggest that the expression of schizophrenia is associated with abnormal fronto-temporal activation during word generation, but that this may also be abnormal in those at high genetic risk of the disorder. 
200P

GENES AND ENVIRONMENT IN THE TRACKING OF BODY SIZE FROM BIRTH TO LATE ADOLESCENCE

Kirsi H. Pietiläinen, Jaakko Kaprio, Maija Räsänen, Torsten Winter,Aila Rissanen, Richard J. Rose

Correspondence address: University of Helsinki, Department of Public Health, Finland.

Background. Body size has been shown to track from birth to adulthood, but it is not known, whether this tracking is due to genetic or environmental effects.

Purpose. 1) To study the patterns of the genetic/environmental architecture on relative weight at birth and in late adolescence, and 2) to estimate the effects of marked discordance in body size at birth on the final size in twins with similar genetic background (monozygotes).

Subjects. A nationally representative sample of Finnish twins (born 1975-1979) with 702 monozygotic (MZ), 724 same-sex dizygotic (SSDZ), and 762 opposite-sex dizygotic (OSDZ) pairs.

Methods. Mailed questionnaires: birth length, weight, and gestational age reported by parents, self-reported height and weight from 16 to $18 \mathrm{y}$. Measures of relative weight: Ponderal index (PI, $\mathrm{kg} / \mathrm{m} 3)$ at birth and body mass index (BMI, $\mathrm{kg} / \mathrm{m} 2$ ) in adolescence.

Results: 1) Twin structural equation (bivariate) modelling revealed that most of the variance of PI was explained by (prenatal) environmental (shared: $17-42 \%$ and non-shared: $38-40 \%$ ) effects, whereas most of the variance of BMI was attributable to genetic effects $(84-90 \%)$. Correlations between the genetic and environmental effects on PI and those on BMI were small (0.1-0.2), but overall, $67-84 \%$ of the phenotypic covariation between PI and BMI was explained by genes. 2) Fetal environmental constraints leading to differences in birth size in MZ twins affected especially the development of height, and, to a smaller degree, that of BMI.

Conclusions. Heritability of relative weight increases with age, and most of the genetic and environmental effects differ pre- and postnatally. However, enduring effects of fetal environment were displayed irrespective of the genetic background. These were stronger for height than for BMI, which seems to be mostly influenced by genes and postnatal behaviour and environment.

\section{S GENETICS AND COGNITIVE ABILITIES AND DISABILITIES}

\section{Robert Plomin}

Correspondence address: Social, Genetic and Developmental Psychiatry Research Centre Institute of Psychiatry, Kings College London, Denmark Hill, London SE5 8AF

rplomin@iop.kcl.ac.uk

At the centre of the nature-nurture debate has been cognitive abilities and disabilities. Decades of genetic research comparing identical and non-identical twins and adoption studies consistently show substantial genetic influence on cognitive abilities such as verbal and spatial abilities and on cognitive disabilities such as reading disability and dementia. Genetic research has now gone beyond simply demonstrating genetic influence to ask questions about relations between cognitive abilities, about development and about the environment. Concerning relations between cognitive abilities, genetic research has shown that the same genetic factors influence diverse cognitive abilities, suggesting that ' $\mathrm{g}$ ' is the genetic core of cognitive abilities. A surprising developmental finding is that genetic influence on ' $\mathrm{g}$ ' increases steadily during the life span. Research at the interface between nature and nurture shows that environmental factors that influence the development of ' $\mathrm{g}$ ' work very differently from the way we thought they worked. An exciting new direction for genetic research is to identify some of the many genes that affect cognitive abilities and disabilities. We have conducted an initial scan of the genome using DNA pooling with two thousand DNA markers in order to find genes associated with ' $\mathrm{g}$ '. Scientific and social implications of finding genes for ' $\mathrm{g}$ ' will be discussed.

\section{P TWIN BROTHERS WITH CONTINUOUS OXALURIA AND RESPIRATOTY OXALOSIS}

\section{Galina Pospekhova, Medeia Mkheidze, Larisa Guleva}

Svetlanovskyi prospect 17-26 194223, St.Petersburg Country Russian Federation mmkheidze@yahoo.com

The condition of a continuous, high urinary oxalate excretion and progressive bilateral oxalate urolithiasis and nephrocalcinosis is a widespread disturbance of St.Petersburg population. Here it is impossible to use specific adequate biochemical and enzyme observations for making diagnosis of hereditary hyperoxaluria I or II. We report on MZ twin brothers with continuous oxaluria. Probands, NN and AN, are 25 years old. Their parents are unrelated. The mother of probands, her parents and probands' father suffered from continuous oxaluria, bilateral urolithiasis and nephrocalcinosis. At birth NN weight was $2100 \mathrm{~g}$, his length was $42 \mathrm{~cm}$ and AN weight was $2200 \mathrm{~g}$, his length was $42 \mathrm{~cm}$.
Now NN weight is $53 \mathrm{~kg}$, his length is $171 \mathrm{~cm}$, AN weight is $58 \mathrm{~kg}$, his length is $170 \mathrm{~cm}$. Their HLA haplotypes are A2-10B13-35. High urinary oxalate excretion was found to be from early probands' childhood. NN has all set of markers of respiratory oxalosis (G. Pospekhova et.al, 1997). AN has mild sings of pulmonary disorder. Both twins have some sings of progressive oxalate urolithiasis and nephrocalcinosis but NN has more severe form of renal disease. Now NN has disability II and AN has disability III as patients with pulmonary disorder. It is obvious that this family needs specific biochemical observation.

\section{S GENETIC CORRELATION BETWEEN INSPECTION TIME AND IO.}

\section{Daniëlle Posthuma, Eco JC de Geus, Dorret I Boomsma.}

Correspondence address: Dep. of Biological Psychology, VUA, Netherlands

Inspection time is the minimum amount of time a subject needs to make an accurate decision on an obvious stimulus, such as the discrimination between two lines. It is part of one of the first, unconscious, stages of information processing. We found a correlation of -0.27 between inspection time and IQ. In order to determine whether this association is mediated by common genes or by common environmental influences, inspection time and IQ were measured in twins and their non-twin siblings. Data from 688 participants from 271 families were collected as part of a large ongoing project on the genetics of adult brain function and cognition. IQ was measured with the Dutch version of the WAIS-3R, inspection time was measured in the so-called $\Pi$-paradigm. Inspection time was moderately heritable $(43 \%)$, whereas the heritability estimate for IQ was high $(86 \%)$. The correlation between inspection time and IQ was entirely due to a common genetic factor which accounted for $14 \%$ of the total variance in IQ. Implications of the existence of a common genetic factor for inspection time and IQ are discussed in terms of a biological model. This model, derived from established theories on visual information processing, proposes that variation in inspection time is dependent partly upon variation in genetic factors that determine myelination and synaptic efficiency in the central nervous system. Thus, $14 \%$ of the interindividual variance in intelligence must be sought in those genetic factors that influence myelination and synaptic efficiency. (The financial support of HFSP grant rg0154/1998-B is gratefully acknowledged)

\section{5}

MEETING THE EDUCATIONAL NEEDS OF MULTIPLE BIRTH CHILDREN ON SCHOOL ENTRY

Pat Preedy

Knowle CE Primary School, Knowle, Solihull, West Midlands. B93 OJE England

This research is from an educational perspective, with the primary purpose of supporting multiple birth children, particularly when they start school. The research methods selected include the first national survey of schools in England and interviews with parents of multiple birth children in one Local Education Authority. The survey produced data from 3,000 schools in 73 Local Education Authorities. The sample consisted of 11,873 twin children, 117 sets of triplets and 5 sets of quads. As only $1 \%$ of schools had a policy with regard to meeting the educational of multiple birth children and only $28 \%$ liaised with parents with regard to separation or keeping multiples together, a framework school policyand questionnaire to be completed in collaboration by teachers and parents has been devised and published on the education website ( $w w w . t w i n s$ and multiples.org). A theoretical model based upon the personal, social and emotional development of multiple birth children has also been developed. The model depicts the potential tension between multiple birth children of being a couple and of being an individual. It is hoped that this model will generate hypotheses and contribute to the development of educational research with regard to multiple birth children.

\section{S}

\section{SEX-SPECIFIC GENETIC INFLUENCES ON THE COMORBIDITY OF ALCOHOLISM AND MAJOR DEPRESSION IN A POPULATION- BASED SAMPLE OF U.S. TWINS}

Carol A. Prescott, Steven H.Aggen, and Kenneth S. Kendler

Correspondence address: Virginia Institute for Psychiatric and Behavioral Genetics, Virginia Commonwealth University, Richmond VA, USA

Alcoholism and depression frequently co-occur but the origins of this comorbidity remain uncertain. Most prior family, twin and adoption studies of these disorders have used cases ascertained through treatment settings, who may differ from cases in epidemiologic samples. We studied the importance of genetic influences on risk for lifetime comorbidity of major depression and alcoholism using a populationbased twin sample. Lifetime major depression (MD), alcohol abuse, and alcohol dependence (AD) were assessed by structured interview for both members of 3,755 twin pairs from the Mid-Atlantic Twin Registry. 
Pair resemblance was analyzed using structural equation models Individuals with MD were at significantly increased risk for AD and for a combined diagnosis of alcohol abuse and/or dependence (AAD). History of MD in a twin significantly increased risk for cotwin AD and AAD among identical male pairs and for AAD in identical female pairs, but not among male or female fraternal pairs. Results of structural modeling indicate that comorbidity occurs because the genetic and specific environmental sources of liability to MD overlap with those underlying AD and AAD. This overlap was significant only within sex, not across sexes. In this population-based twin sample, the familial transmission of major depression and alcohol dependence was largely disorderspecific. Comorbidity appears to be due to sex-specific genetic and environmental risk factors. The factors underlying depression in women do not appear to arise from the same factors underlying alcoholism in men.

\section{P INFANT ZYGOSITY CAN BE ASSIGNED BY PARENTAL REPORT QUESTIONNAIRE DATA}

Thomas S. Price, Bernard Freeman, lan Craig, Stephen A. Petrill, Lorna Ebersole, Robert Plomin

Correspondence address: Social Genetic and Developmental Psychiatry Research Centre, Institute of Psychiatry, London, SE5 8AF, UK

A parental report questionnaire (http://statgen.iop.kcl.ac.uk/ twinzyg.html) posted to a population sample of 18-month-old twins correctly assigned zygosity in $95 \%$ of cases when validated against zygosity determined by identity of polymorphic DNA markers. The questionnaire was as accurate when readministered at 3 years of age, with $96 \%$ of children being assigned the same zygosity on both occasions. The results validate the use of parental report questionnaire data to determine zygosity in infancy. An online version of the questionnaire is available. Twin Research (2000), 3(3), 129-133.

\section{$207 S$ GENE-BY-ENVIRONMENT INTERACTION IN TWIN AND SIB-PAIR ANALYSIS}

\section{Shaun Purcell \& Pak Sham}

Correspondence address: SGDP, Institute of Psychiatry, Denmark Hill, London, UK.

The trait-mediating effects of specific environmental factors can be incorporated into twin and sib-pair analysis, modeled as simple covariates. In sib-pair quantitative trait loci (QTL) analysis, this may increase power to map QTL. Gene-by-environment interaction, on the other hand, represents a moderating effect of an environmental factor upon the expression of a genetic effect (or vice versa) and can also be incorporated into twin and sibpair analysis. Instead of representing the additive genetic variance component, for example, as a single population average, for example. $\mathrm{a}^{2}$, this variance component is expressed as a function of an environmental moderator variable, $\mathbf{M}_{1}$, which might be different for each twin. The expected additive genetic variance component is then dependent on the moderator, i.e. $\left(\mathrm{a}+\beta_{\mathrm{A}} \mathbf{M}_{1}\right)^{2}$, assuming linear gene-by-environment interaction. That is, the genetic effect is partitioned into a mean part, a, and a moderator-linked part, $\beta_{A} M_{1}$. If the moderator has no effect, then $\beta_{A}=0$. The significance of the gene-by-environment interaction can be formally tested by fixing $\beta_{\mathrm{A}}$ to 0 in a nested submodel and comparing model fit. In a completely analogous manner, gene-by-environment interaction can be incorporated in QTL linkage and association models. We explore the efficacy of such models and estimate the power to detect gene-by-environment interactions, with examples from real data.

\section{F FETAL GROWTH AND SYSTOLIC BLOOD PRESSURE IN SWEDISH MALE TWINS IN YOUNG ADULTHOOD}

Finn Rasmussen, Malin Johansson, Bianca De Stavola, David A. Leon

Correspondence address: Division of Epidemiology, Institute of Environmental Medicine, Karolinska Institute, SE-171 77 Stockholm, Sweden.

The aim of this study was to test the fetal origins hypothesis about an inverse association between fetal growth and systolic blood pressure (SBP) in adulthood. The authors studied 921 male twin pairs born between 1973 and 1979 and alive in 1998. Of these pairs 282 were monozygotic (MZ), 398 were dizygotic (DZ) and 241 were non-classifiable from their answers to classical questions about zygocity in a mailed questionnaire. Information about birth weight (BW), birth length, and gestational age (GA) was obtained from the Swedish Medical Birth Registry. Data on height, weight and SBP at ages 17-19 were collected from the Military Service Conscription Register.

Differences in BW within and between pairs were analysed in relation to SBP using random effects linear models. The estimates were adjusted for GA and for age, height and weight at time of examination. The potential confounding effects due to conscription centre and calendar year were also considered. The between pairs effect of $1 \mathrm{~kg}$ increase in BW was $-2.68(-4.95,-0.42)(95 \%$ confidence interval (CI)) for MZ twins, $0.28(-2.35,2.91)$ for $\mathrm{DZ}$ twins, and $-1.68(-3.15,-0.22)$ for all twin pairs. The within pairs effect of $1 \mathrm{~kg}$ increase in BW was $-1.30(-4.15$,
1.54) for $\mathrm{MZ}$ twins, $0.14(-3.49,3.76)$ for $\mathrm{DZ}$ twins, and $-0.21(-2.13$, 1.71) for all twin pairs. The inverse association between BW and SBP observed between pairs of MZ twins may indicate effects of programming in fetal life. The wide CIs for the within twin-pair effects means that no firm conclusions can be drawn about the contribution of genes to the fetal programming phenomenon.

\section{P THE MBF TELEPHONE SERVICE REVEALS THE NEED FOR EDUCATING PROFESSIONALS}

Barbara J. Read, Carole A. Sweetland and Elizabeth M. Bryan.

Correspondence address: Multiple Births Foundation, Queen Charlotte's

and Chelsea Hospital, Du Cane Rd, London W12 OHS, UK

The Multiple Births Foundation's (MBF) Telephone Advisory Service (TAS) was established in 1994. As well as an information and advisory service for professionals, parents and adult twins, it provides a follow up to the specialist MBF paediatric Twins Clinics; consultations with other MBF professional staff; bereavement (and other) counselling. Relevant literature is available for parents and professionals. During a two-year period the MBF TAS received more than 3,000 calls. This study focused on 915 calls referred to one author (BR). These 915 calls came from 420 people and involved 1022 enquiries of which $69 \%$ were identified as common problems such as sleep, behaviour, feeding and language. Appropriately trained professionals should have been able to deal with the majority of these calls in the community. 144 randomly selected clients in this group were sent questionnaires with a $59 \%(85 / 144)$ response rate. Of these replies $68 \%$ had initially sought help from professionals within the community. Only four people felt that they had received helpful advice before contacting the MBF while $83 \%$ of those who had sought help reported it to be unhelpful and/or not applicable to families with multiples. We conclude that there remains a need for education and training on the needs of families with multiple birth children for professionals working in the community.

\section{$210 S$ BACK-NECK PAIN AND SYMPTOMS OF ANXIETY AND DEPRESSION: A POPULATION-BASED TWIN STUDY}

Ted Reichborn-Kjennerud,Espen Røysamb, Camilla Stoltenberg, Jennifer R. Harris

Correspondence address: Department of Psychiatry, University of Oslo, P. O. Box 85 Vinderen, $N$-0319 Oslo, Norway

Pain in the back and the neck are associated with symptoms of anxiety and depression, but the nature of this relationship is not known. Measures of back-neck pain and symptoms of anxiety and depression were part of a self-report questionnaire sent to 3996 twin pairs. Structural equation modeling was applied to determine the extent to which back-neck pain and anxiety and depression share genetic and environmental liability factors. The phenotypic correlation between back-neck pain and anxiety and depression was 0.31 . Univariate analyses revealed that individual differences in anxiety and depression were best explained by genetic and non-shared environmental factors. An additive genetic and nonshared environmental model best explained variance in back-neck pain, but a model with shared and non-shared environmental factors could not be rejected. Bivariate analyses, however, showed that a model with genetic and non-shared environmental factors best explained the correlation between back-neck pain and symptoms of anxiety and depression. The correlations between genetic and non-shared environmental influences for the two phenotypes were 0.45 and 0.20 respectively. There was no evidence of sex-specific effects. These results suggest that the association between back-neck pain and symptoms of anxiety and depression is primarily due to common genetic factors. Non-shared environmental effects also contribute significantly to the correlation, but are mainly specific to each phenotype.

\section{$211 S$ PERINATAL ASPECTS OF THE MULTIPLE PREGNANCY EPIDEMIC}

J Ruiz, R Aurell,JM Mallafré, JM Carrera.

Correspondence address: Department of Obstetrics and Gynaecology, Institut Universitari Dexeus, Barcelona, Spain

Objectives. Review of the perinatal results of the Multiple Pregnancies in our hospital between the 1st of January 1990 and the 31 st of December 2000.

Material and Methods. The espontaneous and induced (ovulation induction, inseminations or IVF) multiple pregnancies in our hospital between 1990 and 2000 are reviewed and the perinatal results such us premature labour, intrauterine growth retardation (IUGR), congenital abnormalities, antepartum haemorrhage $(\mathrm{APH})$, postpartum haemorrhage $(\mathrm{PPH})$, gestational diabetes, caesarean section rates, uterine atonies and perinatal mortality are studied. 
Results. Of the 17706 deliveries in our hospital in the period described above $538(3.03 \%)$ were multiple pregnancies, $481(89.4 \%)$ were twins, $56(10.4 \%)$ triplets and $1(0.18 \%)$ quadruplets. Approximately, $28 \%$ of the multiple pregnancies were IVF pregnancies and $23 \%$ were result of ovulation induction cycles or artificial inseminations. The age range of our patients is between 30 and 34 years. Premature labour occurred in the $47 \%$ of twin pregnancies and in the $100 \%$ of triplets In the last four years we had no triplet pregnancies ending before 28 weeks and the $80 \%$ of them went over 32 weeks. The $18 \%$ of twin pregnancies and the $24 \%$ of triplets had IUGR. Congenital abnormalities appeared in $2.8 \%$ of twins and $9.4 \%$ of triplets.

Conclusion. With the increase in recent years of the multiple pregnancy rates mainly due to the assisted reproduction tecniques, the perinatologists are learning more and more how to decrease the fetal and maternal morbility and mortality. On the other hand, the reproductive medicine specialists are working towards decreasing the multiple pregnancy rates and not to use fetal reduction to solve the problem.

\section{P TWINS' TRUST IN MOTHERS IN CHILDHOOD AND PUBERTY: A HUMAN BEHAVIOR GENETICS PERSPECTIVE}

Atsushi Sakai (Waseda University), Masumi Sugawara (National Institute of Mental Health, Japan), Kazumi Maeshiro (Shirayuri College), Sachiko Amou (Aoyama Institute of Education)

Correspondence address: 2-579-15 Mikashima, Tokorozawa, Saitama, 359-1192, Japan

The purpose of this study was to examine factors that effect on children's trust in mothers. From the point of view of human behavior genetics, we studied the influence of genetic and environmental factors on children' trust in mothers. As part of ongoing longitudinal study in a Japanese twin sample (MZ and DZ, 0-15 years old, total 2,135 pairs), 381 twin pairs (215 MZ pairs, $166 \mathrm{DZ}$ pairs) in childhood and puberty completed questionnaire about trust in mothers and their mothers completed questionnaires about trust in their children and Parenting Bonding Instrument (PBI: Parker et al.,1979). Main results were as follows: First, using genetic ACE (Additive genetic, Common environment, and non-shared Environment) model, the effect of genetic factors on children's trust in mothers increased as children grew, on the other hands, the effect of common environment was decrease. Second, regression analyses showed that the effect of mother's trust in their children on children's trust in their mothers attained significance in both childhood and puberty, but mother's care factor on PBI was only significant with children's trust in their mothers in childhood.

\section{F TWIN PARENTING PATTERNS}

\section{A C Sandbank, B M Sandbank}

Correspondence address: Young Minds, 102-108 Clerkenwell Road, London EC1M 5SA, UK Twins differ from the rest of the population in their dyadic relationship with the rest of their family. This paper looks at the relationship of twins with their own children to determine whether twins as a group share similar parental attitudes. Also examined are the effect of zygosity, birth order, birthweight and dominance on twin parent/child relationships based on questionnaires which look first at childhood relationships, then relationships between the adult and their own children. The last question asks if the parent has experienced problems with setting limits, patience, available time, giving praise, or other. A control group of similar singleton parents has been established. The following early indications are that (a) MZ twins lean towards a helpful and affectionate child (unlike singletons), perhaps mirroring the twin relationship, (b) self-sufficiency was not seen as an attractive attribute by twins, (c) the control group does not favour any attribute uniquely.

\section{STE CONTRIBUTION OF ASSISTED REPRODUCTIVE TECHNOLOGY TO THE RISE IN MULTIPLE BIRTHS}

\section{Laura A. Schieve}

Correspondence address: Division of Reproductive Health, Centers for Disease Control and Prevention, Mailstop K-34, 4770 Buford Highway, NE, Atlanta, GA 30341

Each year, infertility affects millions of couples who desire children Couples have increasingly turned assisted reproductive technology (ART) procedures to overcome their infertility. The first ART birth was in 1978 One public health concern is the impact ART is having on multiple birth rates. To optimize the chance for pregnancy, ART often involves the transfer of more than 1 embryo into a woman's uterus. In the U.S. $>2$ embryos are transferred in $95 \%$ of ART procedures; consequently each year $35-39 \%$ of ART pregnancies and births involve multiple infants. We used the U.S. ART registry to perform a number of studies related to the multiple birth risk. In sum, the findings indicate that 1 in 1997, $0.6 \%$ of the total births, $9 \%$ of the twin births and $>40 \%$ of the triplet and higher order births in the U.S. were conceived with ART;
2) the risk for multiple birth is particularly high among younger women ( $<35$ years) when compared to older women even when number of embryos transferred is held constant; 3 ) younger women who use ART also have a higher likelihood of success and reach peak livebirth rates with fewer embryos transferred than do older women; 4) women who use eggs donated from younger women have high livebirth rates and a high risk for multiple birth regardless of their age; 5) certain procedures used in conjunction with ART may pose an increased risk for monozygotic twinning in addition to dizygotic twinning. These findings will be compared to those from countries such as Denmark and the U.K. that have regulations limiting the number of embryos transferred.

\section{P THE RELATIVE IMPORTANCE OF GENETIC AND ENVIRONMENTAL FACTORS ON VARIATION IN FAT PERCENT - THE IMPACT OF FITNESS}

Karoline Schousboe, Bircan Erbas, John L. Hopper, Kirsten O. Kyvik, Jan E. Henriksen, Thorkild I.A. Sørensen.

Correspondence address: The Danish Twin Registry, SDU-Odense University, Denmark

The aims of this study are: 1) to elucidate the relative importance of genetic and environmental factors on variation in body fat $(\%)$ assessed by body mass index (BMI) and skinfolds (SF), respectively; and 2) to assess the effect of smoking and fitness on the total and the additive genetic variation of body fat(\%). 152 female (79 MZ, 73 DZ) and 103 male (58 MZ, $45 \mathrm{DZ}$ ) healthy twin pairs of 18-57 years underwent anthropometric measuring and a fitness test. Two outcome measures of body fat $(\%)$ were calculated: fat $(\%)_{\text {mI }}$ assessed by BMI and age, and fat $(\%)_{\mathrm{SF}}$ assessed by SF, weight, height, and age. Multivariate analysis methods were applied to these measures of fat $(\%)$ adjusting for age, smoking, and fitness.

Results. Adjusting for age, the heritability of body fat $(\%)$ was about $60 \%$ for females and $74 \%$ for males. Adjusting for smoking, did not alter the total variation of body fat $(\%)$. Adjusting for fitness, reduced the total variation in both fat $(\%)_{\mathrm{BMI}}$ and fat $(\%)_{\mathrm{SF}}$ by more than one third in both females and males. More than two thirds of the reduction of the total variation in body fat $(\%)$ was due to a reduction in the additive genetic variation. However, the fraction of the total variation due to the genetic variation (the heritability) changed very little, in both genders. In summary, smoking does not seem to have much influence on total variation of body fat $(\%)$, whereas the variation in body fat $(\%)$ attributable to fitness exhibits a strong additive genetic effect. This association however, does not change the overall picture of a high heritability of body fat $(\%)$.

\section{P IVF/ART TWIN PREGNANCIES CARRIES HIGHER RISK FOR CESAREAN SECTION}

David Segal, Zahi Ben-Aroya, Michael Friger, Jury Bar-David, Miriam Katz and Moshe Mazor

Correspondence address: Dept. of Obstetrics \& Gynaecology, Soroka University Medical Center, Beer-Sheva, ISRAEL

Objective. To evaluate whether IVF/ART induced twins pregnancies are at greater risk of peripartum complications than spontaneous twins pregnancies.

Study Design. The study population consists of twin pregnancies following ovulation induction and IVF treatment $(n=727)$. The control group consisted of 143 parturients with spontane ous twins pregnancies. Demographic and obstetrical parameters were abstracted retrospectively from the computerized patients' records in our institution during period of 10 years (1989-1998).

Results. Cesarean section was significantly higher in the study group than in the control group $(56.6 \%$ vs. $29.2 \% p<0.001)$. Non-progressive labor during the first stage was higher in the study group than in the control group $(5.6 \%$ vs. $1.2 \%, p<0.0003)$. No statistical significant was found between the two groups as referred to non progressive 2ed stage, abnormal fetal heart rate patterns, abruptio placenta, prolapse of cord, post-partum hemorrhage and vacuum extraction.

Conclusions. IVF/ART induced twin pregnancies appears to be associated with an increased risk for cesarean section, mainly due to non progressive labor at first stage. No other peripartum complications were noted. 
217P NULLIPAROUS PARTURIENTS WITH TWINS PREGNANCIES ARE EXPOSED TO INCREASED RATES OF CESAREAN SECTIONS

David Segal, Zahi Ben-Aroya, Michael Friger, Jury Bar-David, Miriam Katz and Moshe Mazor

Correspondence address: Dept. of Obstetrics \& Gynaecology, Soroka University Medical Center, Beer-Sheva, ISRAEL

Objective. To determine whether nulliparous women with twin pregnancies are at greater risk of peripartum complications than non-nulliparous women.

Study Design. The study population consists of nulliparus women $(n=217)$ whose labor was induced by amniotomy, The comparison group were mulltiparus women $(n=653)$ who were induced by the same way. Demographic and obstetrical parameters were abstracted retrospectively from the computerized patients' records.

Results. Cesarean section rate was significantly higher in the nulliparus group than in the control group $(43.8 \%$ vs. $30.3 \% p<0.001)$. Non-progressive labor during the first stage was higher in the nulliparus group than in the control group $(5.1 \%$ vs. $0.9 \%, p<0.0001)$ as well as nonprogressive labor during the second stage $(4.6 \%$ vs. $0.6 \%, p<0.001)$. Abnormal fetal heart rate patterns were detected during labor in 24 patients $(11.1 \%)$ of nulliparus group, as compared to 34 cases $(5.2 \%)$ in the control group $(p<0.003)$. No statistical significant was found between the two groups as referred to abruptio placenta, prolapse of cord, post-partum hemorrhage and vacuum extraction.

Conclusions. Nulliparus twin pregnancies appear to be associated with an increased risk of cesarean section, mainly due to non progressive labor 1st and 2nd stage and suspicious fetal heart rate patterns.

\section{$218 S$ SOCIAL RELATIONSHIPS: TWINS REARED TOGETHER, TWINS REARED APART AND TWINS WITHOUT PARTNERS}

Nancy L. Segal PhD and Sara Arad

Correspondence address: California State University, Psychology Dept., 800 N. State College Blvd. Fullerton, CA 92834 USA, nsegal@fullerton.edu

The nature and bases of social relationships between twins have fascinated the scientific community for many years. Twin relationships are also meaningful in what they reveal about human social behavior in general. Past research reveals greater closeness between MZ than DZ twin pairs, and more severe bereavement response among surviving MZ than DZ co-twins. This work serves as a backdrop against which to assess findings from the first systematic survey of social relations between twins meeting for the first time as adults. Ratings of immediate and present social closeness and familiarity between reunited twins were explored via a comprehensive Twin Relationship Survey. Responses were available from 89 monozygotic (MZA) and 65 dizygotic (DZA) individual twins and triplets who participated in the Minnesota Study of Twins Reared Apart. Repeated measures analysis of variance indicated a significant main effect of zygosity with MZA twins providing higher mean ratings than DZA twins. Subsequent analyses revealed that a larger proportion of MZA than DZA twins endorsed the highest choice levels of relatedness (e.g., feeling "closer" or "more familiar" - "than best friends") Furthermore, twins' current closeness and familiarity ratings for their newly found co-twins exceeded those for the nonbiological siblings with whom they were raised. These findings are consistent with a variety of theoretical perspectives predicting increased cooperation and affiliation among close genetic relatives, compared to distant relatives and non-relatives.

\section{S WHEN TWINS LOSE TWINS: IMPLICATIONS FOR THEORY AND PRACTICE}

Nancy L. Segal, Ph.D., Shelley A. Blozis, Ph.D., Lauren Sussman, M.S.

Correspondence address: California State University, Psychology Dept., 800 N. State College Blvd. Fullerton, CA 92834 USA, nsegal@fullerton.edu

Factors underlying individual differences in bereavement response have attracted considerable interest. Not all research programs have, however, compared psychological correlates of bereavement across diverse groups of survivors, nor considered findings with reference to a meaningful theoretical framework. Responses from bereaved MZ and DZ twins offer a fruitful domain for testing kinship-genetic predictions generated by evolutionary psychological perspectives. Twin loss studies conducted at CSU Fullerton generally reveal more intense grief intensity (at the time of loss, and currently) and higher scales scores on the Grief Experience Inventory (GEI) (at the time of loss) among surviving MZ than DZ twins. These findings, which have been supported by other investigators, mirror outcomes from twin relationship studies. New analyses, including twins' current responses to the GEI, will be reported. These findings suggest new ways of understanding differential bereavement response within families that may assist counsellors and therapists in devising appropriate intervention strategies. This information should also benefit attorneys and other legal experts representing families in cases of twins' wrongful death and injury.

\section{$220 S$ VIRTUAL TWINS AND WHAT THEY TELL US ABOUT HUMAN BEHAVIOR}

Nancy L. Segal, Ph.D., Samantha Etchells, Nathaniel Houston, Claire V. Bertram.

Correspondence address: California State University, Psychology Dept., 800 N. State College Blvd. Fullerton, CA 92834 USA, nsegal@fullerton.edu

Virtual twins (VTs) are unrelated siblings of the same age adopted into the same family at nearly the same time. These unique siblings pairs replay essential features of twinship, enabling sensitive tests of the contribution of shared environmental influence on behavioural development. This projects has been ongoing at California State University, Fullerton since 1983. An early report, based on 21 pairs, indicated an IQ correlation of .17 , a verbal IQ correlation of -.01 and a performance IQ correlation of .29. New analyses, based on a sample of 90 pairs, yielded an IQ intraclass correlation of $.26(p<.01)$. This correlation, while statistically significant, falls considerably below correlations of $.86, .60$ and .50 reported for monozygotic (MZ) twins, dizygotic (DZ) twins and full siblings, respectively. The VT IQ subtest profile correlation of .08 also falls below corresponding MZ (.45) and DZ (.24) twin correlations. These results are consistent with explanatory models of intelligence that include genetic factors, demonstrating that shared environments have modest influence on intellectual development. Subsequent analyses of VTs' similarity in personality traits and behavioral problems are currently underway. Implications of the findings for theories of intellectual development and for practices regarding rearing and education will be discussed. Future plans for this ongoing study include a broad range of genetically and environmentally informative relationships.

\section{S USING TWINNING TO MONITOR HUMAN FERTILITY}

RogerV Short

Correspondence address: Department of Obstetrics and Gynaecology, University of Melbourne, 132 Grattan Street, Carlton, Victoria. 3053, Australia

Is human fertility declining? Although there has been much concern about a possible decline in the sperm count, animal evidence suggests that the ovulation rate would be the first parameter to be adversely affected if, for example, there were endocrine disruptors contaminating the environment. The ultimate measure of human fertility is fecundability - the probability of conceiving in a given menstrual cycle. Such information is extremely difficult, costly and time consuming to obtain. It is possible to get a good indication of the ovulation rate by studying the incidence of dizygotic twinning, corrected for race and age, and excluding the use of any ovulation inducing drugs or assisted reproductive technologies. Rates are difficult to calculate unless you know the size of the base population, so a simple alternative is to study the ratio of monozygotic (MZ) to dizygotic (DZ) twin births over time. MZ twinning is a near-random embryological event with a similar incidence rate in all human populations, so the MZ:DZ ratio will provide a good indirect measure of the DZ twinning rate. The number of DZ twins born can be estimated quite accurately by doubling the number of opposite sexed twins: this can then be subtracted from the total number of twins born to give the number of $\mathrm{MZ}$ twins. It would be a very simple matter for any country, or large city, to monitor the corrected MZ:DZ ratios over time, and this would provide the best possible early warning system for any significant decline in human fertility.

\section{P FEASIBILITY OF USING DIFFERENT APPROACHES FOR RECRUITING YOUNGER TWINS FOR SRI LANKAN TWIN REGISTRY}

Sisira H Siribaddana, Athula Sumathipala, Devaka JS Fernando, Nimali de Silva, DA Rohana K Dayaratne, Nihal Abeysinghe,S Sivayogam.

Correspondence address: National Twin Registry, Department of Medicine, Sri Jayewardenepura University, Nugegoda, Sri Lanka.

The principle methods of identifying twins for a complete population based register include tracing birth records or conducting community surveys. Our objective was to study the feasibility and effectiveness of different methods to identify and recruit younger twins, to establish a population based register.

\section{Methods.}

(a) To trace twins:

1. Population Survey using an interviewer administered questionnaire

2. Inspecting the birth records at a divisional secretariat (DS), reported from a specified Hospital (Sri Jayewardenepura General Hospital) between the years of 1985-1997.

3. Inspecting birth records between 1985-1997 at the above hospital 
(b) To recruit twins:

A random sample of 75 twins identified at DS was contacted through the post and 25 twins were personally visited to register.

Results. The prevalence of twins was 3.25 twin pairs per thousand people in the area surveyed. The twining rate at the hospital was 9.78 multiple births per thousand live births. A discrepancy of 36 multiples births between the hospital labour room records and those registered at the DS were noted. The response for the postal invitation for recruitment was $59 \%$ and the response for the personal invitation was $71 \%$.

\section{F THE RELATIVE IMPORTANCE OF GENETIC AND ENVIRONMENTAL FACTORS TO DEATH PRIOR TO AGE 65 YEARS}

Axel Skytthe, Niels V. Holm, James W.Vaupel, Knud Juel, Kaare Christensen Correspondence address: The Danish Twin Registry, University of Southern Denmark.

Longevity has been shown to be influenced moderately by genetic factors with a heritability of $0.20-0.25$. However, genetic factors may influence the liability to death differently across different age ranges. For example, in a study of Swedish twins mortality from cardiovascular diseases has been shown to be influenced more by genetic factors at younger age than at old ages. In a Danish adoption study genetic factors were found to play a greater role for death prior to age 50 years than for death at higher ages. Based on a newly established register of Danish twins born 1931-1952 we conducted a classical twin study of the relative importance of genetic and environmental factors on early death, i.e. death prior to age 65 years. Included were 8,349 twin pairs with both members alive on April 1, 1968 and aged 18 years or older. The main findings were that the liability to die prior to age 65 for both men and women are influenced equally by genetic and environmental factors. The findings are consistent whether the analyses were done on the total age range of 18-64 years or were restricted to a narrow age range of 37-49 years. The estimates of genetic effect tended to increase in the younger age range. Furthermore cause-specific analyses have shown that for men a strong genetic component in the liability to die from vascular diseases was present, with a heritability of 0.6 .

\section{P DISCORDANT ANOMALIES IN MONOZYGOTIC TWINS}

Karin Sloots, Renske Rijlaarsdam, Birgit Arabin

Correspondence address: Clara Angela Foundation Zwolle $(N L) \&$ Isala Clinics. Dr.v.Heesweg 2 8025AB Zwolle (NL)

Introduction. Monozygotic (MZ) discordant twin pairs suggest the complex nature of the MZ twinning process.

Methods. We evaluated our data base of monochorionic (MC) twin and dichorionic (DC) triplet pregnancies for discordancy of malformations in MZ multiplets.

Results. In 1999, we registered 5 pregnancies with MZ multiplets discordant for aberrations. All patients had been referred from peripheral hospitals. Case 1: MCDA boys with oligohydramnios/ polyhydramnios and hydrops of the supposed "recipient", the hydropic fetus died 2 days after a CS. Post mortem investigation showed a mediastinal tumor with infiltrative growth into the left lung diagnosed as a sporadic infantile desmoid-like fibromatosis. Case 2: MCMA twins with one girl developing progressive symptoms of hemi-hyperthrophy. DNA examination revealed a Beckwith-Wiedeman syndrome. Case 3: MCDA twins with suspicion of TTTS grade 1, with increasing discrepancy of the head circumference, one girl demonstrating with microcephaly. Case 4: DCDA triplet pregnancy with cord entanglement of the MZ triplets, one of the MZ triplets demonstrated with AVSD and transposition of major blood vessels and abnormal lung-vein drainage and died two weeks post partum. Case 5: MCMA twins with acardius acranius syndrome but intrauterine death of both twins at 13 weeks.

Conclusions. The pathogenesis of discordant MZ phenotyping includes: rupture of the zona pellucida, mosaicism, third type twins, postzygotic chromosomal non-dysjunction, skewed X-chromosome inactivation or abnormal vascular events. This study demonstrates that scanning of all MZ multiplets is obligatory.

\section{F SCHIZOPHRENIA IN TWINS}

Cassandra L. Smith and Giang Nguyen

Correspondence address: Center for Advanced Biotechnology, Boston University,

36 Cummington Street, Boston, Mass 02215. clsmith@bu.edu.

The level of DNA polymorphisms was measured around anonymous $(\mathrm{CAG}) \mathrm{n}$ repeat sites in 12 twin pairs previously classified as monozygotic, and 19 pairs of unselected siblings from 8 families. Eight twin pairs were affected by Schizophrenia: four concordantly and four discordantly. Unexpectedly, four twin pairs, discordant for Schizophrenia had DNA polymorphism levels close to or within the same range as sibling pairs while the other twin pairs had levels greater than sibling pairs. One explanation of this data is that the genomes of Schizophrenics undergo non-random DNA changes at (CAG)n sites. An alternative interpretation is that the twins discordant for Schizophrenia are dizygotic rather than monozygotic.

\section{P HERITABILITY OF BLOOD PRESSURE IN BLACK AND WHITE YOUTH. THE GEORGIA TWIN STUDY}

Harold Snieder, GregoryA Harshfield, Donna Moore \& Frank Treiber

Correspondence address: Georgia Prevention Institute, Medical College of Georgia, Augusta, GA, USA

Twin studies of blood pressure (BP) have found heritabilities around $50-60 \%$ for both systolic (SBP) and diastolic blood pressure (DBP), but have mostly involved Caucasian twins. Mean levels of BP are known to be higher in blacks, but relatively little is known about ethnic differences in genetic and environmental influences on BP.

We studied 308 white and 226 black twin pairs from the south-eastern United States including monozygotic (MZ) and dizygotic (DZ) of sameas well as opposite-sex (mean age: $14.7 \pm 3.1$; range: 10.0-25.9). Supine resting SBP and DBP were measured three times (Dinamap) and averaged over the last 2 measurements. Mean values were adjusted for the effects of age, sex and race and their interactions. Race and sex effects on genetic and environmental contributions to BP were estimated by genetic model fitting. For SBP, the best fitting model showed no differences in heritability between blacks and whites or males and females $\left(\mathrm{h}^{2}=0.57,95 \% \mathrm{CI}: 0.49-0.63\right)$. Heritability of DBP was 0.45 (95\%CI: $0.34-0.56)$ in whites and $0.58(95 \% \mathrm{CI}: 0.45-0.68)$ in blacks with no effect of gender. The effects of shared environment and dominance were not significant for SBP or DBP. In summary, heritability estimates for SBP and DBP in black youth are similar to those in white youth and consistent with previous research in Caucasian twins.

\section{F THE ITALIAN TWIN REGISTRY}

M. Antonia Stazi, Rodolfo Cotichini,Valeria Patriarca, Cristina D'Ippolito, Marco Salvetti, Giovanni Ristori, Stefania Cannoni, Roberto Tosi Correspondence address: Istituto Superiore di Sanità, Rome, Italy

In 1997, starting from the social security number database of the Italian Ministry of Finance and using a record linkage procedure based on last name, place and date of birth, the entire population of potential Italian twins alive at the end of the previous year was identified. This strategy allowed the detection of about 800,000 potential twin pairs and multiples. A check on the completeness of the register was performed in four municipalities: we were able to trace $80 \%$ of pairs in which one or both the twins lived in the municipalities. The remaining $20 \%$ was comprised of non-sib individuals or due to double-attribution of the social security number to the same individual. We estimated approximately $1,200,000$ twins alive in Italy in 1996. The register is currently being updated and new addresses will be recorded to facilitate tracking; a system for linking mortality data has also been implemented. The Italian Twin Registry is supported by a project grant from the Health Ministry, which is administered by the National Institute of Health. In 2001, 70,000 twin pairs (three cohorts: children 6-10, adults 35-40 and 65-70) will be asked by a mail questionnaire to be formally involved in the register, for taking part in medical and health research. The effectiveness of this enrolment procedure is under testing in a pilot study. The registry is intended to be a resource for scientific and medical researchers. A biological specimen bank from twins of different ages will be created, guaranteeing information on etiological questions regarding genetic and modifiable factors for illnesses.

\section{$228 \mathrm{~F}$}

\section{ASSESSING NEED AND DEVELOPING A STRATEGY FOR A NATIONAL VOLUNTARY} ORGANISATION TAMBA.

\section{David Stern}

Correspondence address: 3, Eliot Vale, Blackheath, London SE3 OUU

This presentation will outline the process used to develop a strategy for the Twins and Multiple Births Association (Tamba). The history, growth and development of Tamba was examined and how the organisation met the needs of families with twins, triplets or more. The review led to the development of a 3 year strategy and plan of action. The presentation will illustrate the historical performance, vision, and values. The achievements and existing services of the organisation were examined. The strategy for the future included activities and priorities, provision of support services and specialist groups, awareness raising, external communication, and fundraising. The operating structure and support systems were given further consideration in order to identify gaps, implications and make recommendations. An action plan was developed with specific targets. Tamba is currently implementing the plan and is regularly monitoring and evaluating progress and the way forward. 


\section{F THE SOCIAL REALITY OF TWINSHIP}

\section{Elizabeth A. Stewart}

Correspondence address: Department of Sociology, London School of Economics, Houghton Street, London WC2A $2 A E$, England

Within the twin context, interaction with others particularly stresses and reinforces 'twinship'. Therefore being — and remaining — a twin carries social significance. Social processes external to the twin unit may emphasize the unitary nature of twinship; to the extent that this is the case, the negotiation of twinship by the relevant twins both within wider social groups and between themselves will be coloured by such processes. Statements such as 'I am a twin' or 'Oh, you are twins, are you?' are actually statements about lack of individuality and lack of autonomy. The social analysis of twinship must address both the culturally variable construction of 'twinship' (what twins 'are' within particular cultures and what norms they are expected to conform to) and how twins variably and creatively negotiate the pressures of a unitary identity, whether in terms of compliance, contestation or denial. The usual processing of members of Western societies takes place in unitary terms, whereas twins have to confound expectations and assumptions by others about cloning, identical behaviour and lack of individuality. This presentation further locates twinship as a minority status within the social construction of childhood and argues for an analysis of childhoods rather than childhood. Finally, identity formation of twins is considered as different for twins in regard to maternal relations, to the need both to separate and differentiate, to confront statements about lack of individuality, and to become an individual in an increasingly individualized culture.

\section{F SYSTEMATIC ASCERTAINMENT OF TWINS WITH CLINICALLY APPARENT DISEASE IN BRITISH GENERAL PRACTICES: A PILOT STUDY}

\section{David P. Strachan, Beverley Botting, Madge R.Vickers}

Correspondence address: Department of Public Health Sciences, St George's Hospital Medical School, Cranmer Terrace, London SW17 ORE, U.K.

A computerised search of the National Health Service (NHS) Central Register has generated lists of probable and possible twins alive and resident in England and Wales in April 1991. The twin status of the possible pairs may be determined more precisely by reference to birth registers but this clerical work is contemplated on an incremental basis, as relevant to individual research projects. This nationwide database of twins and higher order multiple births, containing 2,155,010 persons, cannot be used directly to contact individuals, but can be used to identify probable and possible twins on other lists. We carried out a pilot study to evaluate the feasibility of identifying twins attending seven Medical Research Council research practices with a combined list size of 75,212 patients. The patient registers from each practice were matched against the nationwide twin database at National Statistics. 861 (1.1\% of patients) were identified as "probable twins" on the basis of NHS number and $836(97 \%)$ of these were clerically confirmed as twins in birth registers. An additional 2102 patients (2.8\% of the list) had NHS numbers indicating they were "possible twins", and $154(7 \%)$ of these were confirmed in birth registers. The medical records of the "probable" twins were reviewed and summarised in anonymised form by the research nurses in each training practices. 93\% (799/861) records were traced. This pilot study demonstrates the feasibility and efficiency of record linkage for identifying diseased twins in Britain.

\section{P THE PERINATAL OUTCOME OF THE CO-TWIN OF MONOCHORIONIC TWINS WHEN ONE FETUS DIED IN UTERO}

Noriyuki Suehara, Kenshi Wasada, Takasi Sugiyama Takahiro Mizutani, Masanori Fujimura, Masahiro Nakayama and Kimiyo Suehara Correspondence address: Department of Obstetrics, Osaka Medical Center and Research Institute for Maternal and Child Health. 840, Murodou-cho, Izumi-city, Osaka, 594-1101 Japan.

We surveyed 33 liveborn monochorionic co-twins where one fetus had died in utero in Osaka Medical Center during 1981 to 1999. Among the 33 liveborn co-twins, 12 died and 5 have cerebral palsy. Poor prognostic factors for co-twins include 7 cases complicated by embolism, 6 cases with acute twin-to-twin transfusion syndrome (TTTS) and 6 cases with chronic TTTS and 7 neonates were born before 26th gestational weeks. Among 7 cases with embolism 6 cases had venous-venous anastomoses on the surface of the placenta. All cases were born some days after the intrauterine death of the other fetus. We propose that early delivery (before 3 days) after one fetal death should protect the twin embolism syndrome of MC twins. Acute TTTS (Reversed TTTS) may result in hypoxic-ishemic change following severe asphyxia and severe anemia. Acute TTTS may occur in the co-twins delivered immediately afte the intrauterine death of the other fetus. Among 10 cases with acute TTTS, there were 5 neonatal deaths, one case of cerebral palsy and
4 healthy survivors. 6 cases were complicated with chronic TTTS Hypertrophic heart failure in the larger fetus was an important cause of neonatal death, PVL and cerebral palsy. Among 11cases with chronic TTTS, there were 6 neonatal deaths, one case of cerebral palsy and 4 healthy survivors. 6 cases were complicated with acute TTTS.

\section{P DEVELOPMENTAL PSYCHOPATHOLOGY: A BEHAVIOURAL GENETIC APPROACH}

Masumi Sugawara, Atsushi Sakai, Kazumi Maeshiro, Sachiko Amou Correspondence address: National Center of Neurology and Psychiatry, 1-7-3 Kohnodai, Ichikawa, Chiba 272-0827, Japan. sugawara@ncnp-k.go.jp

The purpose of this study was to investigate contributions both of genetic and environment al factors to development of children's personality and psychopathology. As part of ongoing longitudinal study in a Japanese twin sample (MZ and DZ, 0-15 years old, total 2,135 pairs), a questionnaire survey of mothers of 706 pairs of school-aged (aged 7-15) children and 956 pairs of preschool children (aged 2-6) was administered. Children's behavioural characteristics was assessed by Cloninger's Temperament and Character Inventory (TCI, junior version and preschool version), and problem behaviours and psychiatric symptoms were measured by Achenbach's Child Behaviour Checklist (CBCL) and a selfrating depression scale. ACE model analysis (A: additive genetic factors, $\mathrm{C}$ : shared environmental factors, E: non-shared environmental factors), yielded two major conclusions. First, temperament in preschool period and childhood was influenced by additive genetic factors and non-shared environment, like as adult personality trait, the effect of common environment was hardly observed. Second, approximately $20-50 \%$ of the variance in problem behaviours (internalizing and externalizing problems) was due to common environment factors. A developmental change of contributions of genetic and environmental factors about TCI, CBCL, and children's depression will be presented.

\section{P HIGHER ORDER MULTIPLE BIRTHS IN THE UNITED STATES: A SNAPSHOT AT THE TURN OF THE CENTURY}

\section{Kate Sullivan Collopy}

Correspondence address: MOST (Mothers of Supertwins), P.O. Box 951, Brentwood, NY 11717-0627 USA

This paper presents a descriptive analysis of pregnancy, birth, and neonatal data from over 1,000 higher order multiple pregnancies. The study included participants from 45 out of the 50 United States as well as Canada, Mexico, Ireland, England, Israel, and Greece. The majority of subjects conceived their higher order multiples (HOM's) through IVF $(32.5 \%)$. Other methods of conception included the use of ovulationinducing medications alone $(22.8 \%)$ and intrauterine insemination $(18.1 \%) .88 .5 \%$ of the respondents delivered triplets, $9.6 \%$ quadruplets, $1.7 \%$ quintuplets, and $0.2 \%$ sextuplets. The mean maternal age was 31.5 years, with a range of 20 from 46 years. $74.4 \%$ were hospitalized at least once prior to delivery (with the first hospitalization at a mean of 24.6 wks.). $54.1 \%$ of these admissions were due to preterm labor. The antenatal length of stay ranged from less than one day to 105 days, with a mean of 15 days. $55.6 \%$ of all respondents reported preterm labor (with a mean gestational age at onset of 25.8 wks.). $15.2 \%$ experienced premature rupture of membranes (mean gestational age $29.4 \mathrm{wks}$.). The reported mean age at delivery for triplets was 33.4 weeks (ranging from 16.0-38.5), quadruplets, 31.0 weeks (ranging from 23.0-37.0), quintuplets, 28.6 weeks (ranging from 19.5-34.0), and sextuplets: 30.0 weeks (ranging from 29.0 to 31.0). Maternal weight gain ranged from 0-110 lbs., with a mean gain of $51.4 \mathrm{lbs}$.

\section{PLAYING GOD: A PHENOMENOLOGICAL STUDY OF WOMEN'S DECISION-MAKING REGARDING MULTIFETAL REDUCTION}

\section{Kate Sullivan Collopy}

Correspondence address: University of New Hampshire, 257 Hewitt Hall, Durham, NH 03824 USA

This study explored women's experiences regarding their decisions to undergo or forgo multifetal reduction. The purposive sample consisted of seven women who had conceived higher order multiple pregnancies as the result of in vitro fertilization. Four participants had chosen to undergo reduction while three participants opted to forgo reduction. Van Manen's (1990) phenomenological method was used for data collection and analysis. Participants were asked to respond to the question, "What was it like to make the decision whether or not to reduce your higher order multiple pregnancy?"

Five themes emerged from the data: 1) All You Can Think About is Having a Baby: in which the participants described a single-minded, relentless pursuit to conceive; 2) There are Three: where the participants described emotions ranging from concern to euphoria upon receiving the diagnosis of higher order multiple pregnancy; 3) It's Like BOOM 
and You Have to Decide: where the participants told of having to make a difficult, life-altering decision in a very brief time period; 4) We Didn't Deserve This: in which the participants described experiencing multiple layers of loss while attempting to become mothers; and 5) What If / Which One?: where the participants described the lasting effects of their decision, wondering about ways in which their pregnancy outcomes might have been different or which one of their children might have been reduced.

\section{S STARTING FROM SCRATCH IN SRI LANKA}

Athula Sumathipala

Correspondence address: SEGP, Institute of Psychiatry, London.

Commitment against obstacles and basic knowledge was vital, but books and journals were a scarcity. This demanded networking with experienced twin researchers. A partnership model for collaboration was used for mutual scientific benefits but not for economically cheaper research in the developing world. Forming a multi-disciplinary team was essential, but for locals twin research was new. Funding was hardest without prio twin research. We were compelled to seek help from commercial establishments but were aware of conflict of interests. Therefore, guidelines were developed on bioethics, consent, data collection, storage and access to the twin database and human biological material, funding, commercia exploitation, international collaboration, and authorship to safe guard the Sri Lankan interests as the moral "rights" and "wrongs" are not absolute but vary with the culture. We initiated a volunteer register launching a competition through media. 4684 multiples registered. Most were less than 30 years. Adaptation and preliminary validation of a zygosity questionnaire was carried out. Using birth records to trace younger twins were feasible, but encountered many problems for older twins. Use of different strategies to establish a population-based registry is possible as evident by feasibility studies. Twins born in the whole island from 1992 are available to us through the Department of Birth Registration. We are now supported by a grant from the Wellcome Trust. Newsletters for twins and researchers are published. Local capacity building is in progress. A Multiple Birth Organisation was formed to represent the interests of multiples and to raise awareness on their issues. It will work with professionals, statutory organisation to initiate service development.

\section{F THE KOREAN TWIN REGISTRY}

Sung J, Duffy DL, Cho S-H, Moon S-Y, Cho SI,Yoo KY, Kang DH

Correspondence address: Dept of Preventive Medicine, Kangwon National University

Although twin study can be especially useful to discriminate between genetic and environmental causes of human diseases, most study has been undertaken in Western countries. The Korean Twin Registry is the first nationwide twin study in Korea. It started from the lists and health outcomes of twins gathered through a serial data linkage among nationwide data sources: address code data; Korean National Health Insurance Medical Utilization and Insured Family data; and birth report and death certificate data. Uniform 13-digit ID system, applied to all Koreans from birth registry until death, enabled exact data matching among various sources. The registry totals 156,472 , born from 1956 . Comparing with the number in birth report data (available since 1982), the registry coverage $1982-1996$ was $93.1 \%(104,237$ out of 111,911$)$ ranging from $86.4 \%$ (1986) to $99.9 \%$ (1996)/ The registry coverage for twins born between 1979 and 1996 was estimated to be more than $90 \%(125,779)$. The list of twins born after 1997 is being updated, and expected to reach as complete level. However, for those born before 1979, completeness drops sharply. Medical utilization data from seven major diseases since 1991 was collected. Individual death certificate data since 1992 was available and case ascertainment of registered twins is being undertaken. The first target diseases were congenital malformations and asthma. Despite some strengths in size, population representativeness, and readiness to ascertain some pediatric diseases, we have not yet conducted surveys on the zygosity and basic risk factors but these are planned. Analyses using disease concordance rate between like-sex and opposite-sex twins are already ongoing.

\section{S}

\section{MONOZYGOTIC TWINNING AND ASSISTED REPRODUCTION}

\section{Alastair G. Sutcliffe}

Correspondence address: Academic Dept. of Child Health, Royal Free Campus, Royal Free and University College Medical School, Rowland Hill Street, London, NW3 $2 P F$ icsi@rfc.ucl.ac.uk

A recent review (Sills et al., Twin Research (2000) 3, 217-223) of possible processes which may contribute to and explain the observations of higher rates of monozygotic twinning after ART was inconclusive as to whether ART per se has a true role in this apparent phenomena. From early in the development of IVF observations by McDonough and others proposed the first known 'cause' of an increase in monozygotic twinning. Initially this was thought to be as a bi-product of the use of powerful gononadotrophic regimes. But no exact mechanism was forthcoming. More recently due to advances in, and development of, more invasive ART techniques, specific mechanisms have been proposed as to why MZ twins may be more common. These will be reviewed and will include intracytoplasmic sperm injection (ICSI), extended embryo culture, assisted hatching methods (using acid tyrode and more recently laser techniques.) Also the possible implications of the use of Preimplantation Genetic Diagnosis, which involves the process of embryo biopsy will be discussed.

\section{COMPUTER ASSISTED TELEPHONE INTERVIEWS WITH 26,000+ TWINS: A NEW UPDATE} OF THE SWEDISH TWIN REGISTRY

\section{Pia Svedberg, Nancy L.Pedersen, Paul Lichtenstein}

Correspondence address: Dept Medical Epidemiology, The Swedish Twin Registry, Karolinska Institutet, Box 281, SE-171 77 Stockholm, Sweden.

The Swedish Twin Registry comprises in principle all twin births in Sweden since 1886. All living twins in the Swedish Twin Registry born before 1958, are currently contacted for screening of health status. This current update of the registry began in 1996 with a pilot study that included a random sample of twin pairs for each birth year between ages 17 to 85 . All twins were contacted for a telephone interview screening for health status. During 1998, the health screening was expanded to a larger scale and by January 2001 , more the 26,000 individuals have been contacted. All twins born before 1940 have been contacted and by the end of 2001, all twins born before 1959 will be contacted. Most recent information on last name and address is linked to the telephone company's files to obtain telephone numbers. Introductory letters describing the study are sent to a random sample of approximately 1,000 pairs each month. All data collection is performed with a computer assisted telephone interview by trained interviewers with relevant medical competence. The interview includes a number of items asked to all twins regarding different diseases and symptoms. All administrative data as well as all data collected through screening are entered into an object-oriented database. A series of web-based pages with secured access have been designed to facilitate online administration.

\section{GENETIC AND ENVIRONMENTAL CONTRIBUTIONS} TO RECURRENT HEADACHE

\section{Svensson, N Pedersen, K Ekbom, B Larsson, EWaldenlind.}

Correspondence address: Division of Neurology, NEUROTEC, Karolinska Institutet, Huddinge University Hospital, S-4186 Huddinge, Sweden.Dan.Svensson@neurotec.ki.se.

Recurrent headache (RH) is a very common health problem in the population. A nationwide population of 55 to 64-year-old twins were interviewed by trained lay personnel on the telephone about common health problems across life span including $\mathrm{RH}$. Items regarding headache symptoms permitted diagnoses in line with consensus criteria of migraine and tension-type headache. Twins were also asked if they themselves thought they had ever had migraine - self-reported migraine. The genetic and environment contributions to liability for different types of headache were computed for 2,053 complete twin pairs. Estimates of the lifetime prevalence and the heritability are listed.

\section{S HOW FAMILIES COPE WITH PERINATAL LOSS OF A TWIN*}

Patricia B. Swanson

Correspondence address: Curtin UT; GPO Box U1987; Perth, WA 6845 Australia

Western Australia has one of the most comprehensive databases in the world for the study of multiples. The Western Australia Twin Child Health study (WATCH) has compiled data on all multiples born in the state since 1981 , including 476 perinatal deaths. Their mortality rate is 4-6 fold that of singletons. Parents with multiples are more likely to experience the joy of a newborn simultaneous with the grief of a death. Little is known, through systematic studies, about the psychological impact of these special losses on families. Woodward (1998) concluded from a study of 200 adult volunteer lone twins, that $81 \%$ felt their loss to have a "severe" or "marked" effect on their lives. Segal (1995) using the Grief Experience Inventory (GEI) on subjects over 15 years found twins' grief scores significantly exceeded those of 102 bereaved singletons. But what is the impact on younger surviving twins and three other subsets of the family: mums, dads and siblings? Through interviews with parents and children, this study (currently in progress) aims to:

- Learn how families are impacted by a twin's death

- Generate information to help families cope with the reality of their twin's death

- $\quad$ Provide professionals with information related to what multiple birth families express they need 
- Create written materials directly addressing the needs of families coping with loss of a multiple

- Develop written materials useful to families expecting multiples, and to those who are involved with them.

*The term "twin" includes all multiples.

\section{P WHEN IS A TWIN NOT A TWIN*?}

\section{Patricia B.Swanson}

Correspondence address: Curtin UT GPO Box U1987; Perth,WA

With the advent of Assisted Reproductive Technology (ART) some sad queries are heard more often these days: "Mummy, am I still a twin?" the surviving multiple asks, while bereaved mothers and fathers wonder, "Are we still considered parents of twins?" Modern medicine has led us reason to expect babies not only to survive the womb but also to thrive after birth. But the substantial database of Prof. Fiona Stanley at the Institute of Child Health Research and the Western Australia Twin Child Health shows the mortality rate of multiples is 4-6 fold that of singletons.

What is the psychological impact of twin loss on families? How do families accommodate to their losses? How is this loss different for the surviving twin, siblings and parents? Is it different from loss of a singleton? How long does it take for a family to recover? Using the WATCH database, we contacted and interviewed 70 bereaved multiple birth families in Western Australia. The majority of participants have been mothers, followed by fathers, siblings and co-twins. The study was both qualitative and as well as quantitative: Parents completed The Beck Depression Inventory (BDI-II) and the Perinatal Grief Scale when appropriate. The 200 interviews represented families' losses at any time during the twins' lifetimes. These bereaved families are providing insights to help us not only support them, but also develop materials for them and for professionals concerned with their care.

Rotary International and Curtin University of Technology have supported this research.

*The term "twin" includes all multiples.

\section{S EPIDEMIOLOGICAL STUDIES OF CANCER IN TWINS IN ENGLAND AND WALES}

Anthony Swerdlow, Institute of Cancer Research

Correspondence address: Section of Epidemiology, D Block, Cotswold Road, Sutton, Surrey SM2 $5 N G$

Large-scale epidemiology of serious chronic diseases in twins in Britain is made difficult by the lack of a long-term national twin register. The presentation will discuss a study in which twins with cancer in England and Wales were identified and the patients were interviewed about zygosity and potential factors for cancer. Results on risks of cancer in twins will be presented, with regard to risks by birth order and sex of co-twin, risks in co-twins of individuals with breast and testicular cancers, and case-control comparisons in discordant pairs of risks in relation to environmental and behavioural factors.

\section{$243 S$ FUNCTIONAL STUDIES OF MONOCHORIONIC ANASTOMOSES IN VIVO}

Myles J.O.Taylor, Ling Wee, Philip M Cox, Nicholas M. Fisk

Correspondence address: Queen Charlotte's and Chelsea Hospital, London, W12 ONN

Advances in technology have enabled the study of monochorionic (MC) placental anastomoses in vivo. Anastomoses have been identified invasively at endoscopy, and non-invasively by Doppler ultrasound. Data from an ex vivo model suggest that up to $50 \%$ of arterio-venous anastomoses (AVAs) may be missed endoscopically. The attraction of non-invasive anastomotic research is that the findings can be validated at postnatal injection study and in vivo functional studies are also permitted. Arterio-arterial anastomoses (AAAs) are detected with an overall sensitivity of $85 \%$ and a specificity of $97 \%$. Detection of an AAA reduces the odds of developing twin-twin transfusion syndrome (TTTS) by a factor of 9 and significantly improves outcome in the event that TTTS develops. Unlike AAAs, veno-venous anastomoses (VVAs) have been demonstrated to transmit unidirectional blood flow.

Deep AVAs have been detected using colour Doppler but detection rates are poor, being strongly influenced by vessel diameter, gestation and placental site. Indirect assessment of single AV anastomotic flow rates (derived from AAA counter blood flow measurements) have been complemented by direct assessment of AV flow, obtained from larger calibre vessels.

The use of 3D colour Doppler has further enabled a greater detailed understanding of MC placental anatomy. These techniques can be used to assist endoscopic treatment of TTTS and may ultimately lead to less invasive treatments. ABILITY LEVELS IN ADOLESCENTS*

Yulia Tchertkova, Svetlana Piankova,

Correspondence address: Psychological institute of RAE, Mokhovaya st., 9 " V", Moscow, Russia, 103009

We investigated the possibility of differential heritability of IQ across ability levels in adolescents. 118 pairs of monozygotic (MZ) and 91 pair of same-sex dizygotic (DZ) twins aged 12.5-14 years were tested, using WISC. 3 groups were distinguished: 1) low-ability group (26 pairs of MZ and 15 pairs of DZ twins), 2) average-ability and 3) superior-ability group (21 pair of $\mathrm{MZ}$ and 22 pair of $\mathrm{DZ}$ twins). The intrapair correlation in IQ was 0.77 for MZ and 0.62 for DZ twins, measured for all the sample, so the estimate of heritability was 0.30 . For the low-ability group $\mathrm{H}^{2}=0.19$ and for the high-ability -0.22 , that is slightly lower than for the medium-ability group $\left(\mathrm{H}^{2}=0.32\right)$. Heritability of verbal IQ (VIQ) shows the same regularity. The estimate of heritability for the performance IQ much higher in the average $\left(\mathrm{H}^{2}=0.52\right)$ and superior-ability $\left(\mathrm{H}^{2}=0.40\right)$ groups, than in low-ability $\left(\mathrm{H}^{2}=0.02\right)$ group. A possible explanation for this is a genotype-environmen tal correlation: persons with higher ability seek out better environment.

* Data of the Moscow Longitudinal Twin Study

\section{S THE EMOTIONAL WELL-BEING OF MOTHERS OF TWINS}

Karen J Thorpe

Correspondence address: Dept. Psychology, University of the West of England, Oldbury Court Rd, Fishponds Bristol BS16 2JP

The care of twins presents additional and exceptional stresses compared with those experienced by parents of singletons. This may have an adverse effect on the emotional well-being of mothers of twins. The paper presents two studies which sought to examine the association between child care burden and the emotional status of mothers. These studies were particularly focused on the number and spacing of children within a family and the impact of having twins.

Both studies are analyses of data from large birth cohorts: (1) the Child Health and Education Study and (2) Avon Longitudinal Study of Pregnancy and Childhood. In both, standard measures of the emotional well-being of the mother were made and the impact of number and spacing of children, including twinning, assessed. Analyses controlled for social class, maternal age and education, mother's work and marital status and whether or not the pregnancy was planned. Results indicate that both mothers of twins and those with singletons spaced less than two years apart have raised risk of experiencing depression and that mothers of twins had the highest mean scores on depression scales.

Discussion centres on the impact of childcare burden on emotional well-being of mothers.

\section{P THE EFFECT OF LOSING THE TWIN AND LOSING THE PARTNER ON MORTALITY}

Cecilia Tomassini,Alessandro Rosina, Francesco Billari,Axel Skytthe, Kaare Christensen

Correspondence address: Age Concern Institute of Gerontology, King's College, London, UK. cecilia.tomassini@kcl.ac.uk

Several studies have explored the impact of marital bereavement on mortality, while increasing emphasis has recently been placed on genetic factors influencing longevity. In this paper, we study the impact of losing the spouse and losing the co-twin for adult twins. We use data from the Danish Twin Register (with data on more than 30,000 Danish twins born between 1870 and 1950) and the Population Register of Denmark. Firstly, we use survival analysis to study mortality after the death of the spouse or the co-twin. We find that the risk of dying is highest in the first year after the death of the spouse, as well as in the second year after the death of the co-twin. We then use event history analysis techniques to show that there is a strong impact of the event 'losing the co-twin' even after controlling by age, sex and zygosity, and that this effect is significantly higher in the second year of bereavement. The effect is similar for men and women, but it is significantly higher for monozygotic twins. The effect of losing the co-twin on mortality has been explained so far mainly in terms of genetic factors, as it is well known that the survival of twins is correlated by the action of common genes. Our findings suggest that there is not just correlation, but also interdependence between the two deaths, and this effect is stronger for identical twins. Our results are consistent with the existence of both a genetic and a twin bereavement effect. 


\section{SELECTIVE TERMINATION OF ACARDIAC/ ACEPHALIC TWIN BY RADIOFREQUENCY ABLATION}

KuoJen Tsao, Vickie A. Feldstein, Per L. Sandberg, Roy A. Filly, Craig T.Albanese, Michael R. Harrison

Correspondence address: Department of Radiology, Box 0628, University of California, San Francisco, CA, USA 94143-0628

Acardiac/acephalic twin malformations are a rare anomaly. The normal "pump" twin perfuses the acardiac/acephalic twin resulting in twin reversed arterial perfusion (TRAP). Various technique s have been attempted to obliterate the vascular connections between the donor ("pump") and acardiac twins. Seven cases in which the blood flow to the acardiac twin was obliterated by radiofrequency ablation (RFA) are reported. Patients were evaluated by means of ultrasound (US) with Doppler. Gestational age at time of intervention ranged between 17 and 24 weeks. Under real-time US guidance, a 14 gauge RFA device was inserted through the maternal abdominal and uterine wall, into the abdominal wall of the acardiac twin, at the level of the cord insertion. RFA was initiated after adequate placement of the device. RFA was discontinued once cessation of blood flow to the acardiac twin was achieved, as documented by US. All 7 patients and pump fetuses tolerated the procedure without major complications. On post-procedure US, blood flow was obliterated in all acardiac fetuses and donor twins appeared normal. To date, 4 patients have delivered infants without sequelae. Two patients remain pregnant. One patient delivered an infant at 24.4 weeks which subsequently died from complications of prematurity. TRAP sequence is a rare anomaly that compromises the pump twin. Percutaneous RFA with US guidance is a minimally invasive technique that effectively obliterates the blood supply to the acardiac twin in order to protect the normal co-twin.

\section{P COMPARATIVE RATING MEASURES OF HEALTH AND ENVIRONMENTAL EXPOSURES: HOW WELL DO TWINS AGREE?}

Adam M. Turner, Chandra A. Reynolds, Nancy L. Pedersen, and Margaret Gatz. Correspondence address: University of Southern California, Department of Psychology, Los Angeles CA 90089-1061 USA.

Epidemiological studies often compare one twin's self-reported behaviors and exposures to the twin partner's in order to test whether the exposure is related to a health outcome experienced differentially by the twin pair. An alternative technique is to use comparative ratings, in which twins are asked if they or their partner had greater exposure. The present study asks: (1) to what extent do twin pairs agree on comparative ratings? (2) how well do ratings agree with objective information about exposures? Using $55 \mathrm{MZ}$ and $71 \mathrm{DZ}$ pairs reared together, percentage agreement was calculated for 12 comparative ratings (including agreement on which member was more exposed, or that both were equally exposed). Pairs agreed on average about half of the time (MZ, 52\%; DZ, $48 \%$ ). Agreement was best for more discrete exposures, such as head injury $(72 \%)$ and lowest for more subjective or private variables, such as degree to which life is experienced as stressful $(30 \%)$. Signed rank tests were used to contrast comparative ratings to differences in selfreports, e.g., whether the twin rated as the heavier smoker had previously self-reported more tobacco use. For variables where self-report indices were available, the twin identified by the comparative ratings differed significantly from the twin partner in the direction suggested by the rating. Comparative ratings appear most accurate for smoking and alcohol use, and less consistent for mental health symptoms and self-rated health. This research was supported by NIH grants No. R01-AG08724 and AG10175.

\section{P}

\section{BIRTH ORDER AND ADULT SIZE} AND BLOOD PRESSURE IN TWINS

Chuluuntulga Tuya, Geraldine McNeill, Doris M.Campbell.

Correspondence address: Faculty of Medicine and Medical Sciences,

University of Aberdeen, Aberdeen AB25 $2 Z D, U K$

We have studied the relationship between birth order, adult size and blood pressure (BP) in $60 \mathrm{MZ}(22 \mathrm{M}$ and $38 \mathrm{~F})$ and $70 \mathrm{DZ}$ same sex (30M and 40F) twin pairs aged 33 (range 18-50) years recruited via obstetric records. Gestational age was on average 37 (range 28-44) weeks. The table shows data for first and second born twins:

\begin{tabular}{|c|c|c|c|c|c|c|c|}
\hline \multirow[t]{2}{*}{ Variable } & & \multicolumn{2}{|c|}{ All $N=130$} & \multirow{2}{*}{\multicolumn{2}{|c|}{$\begin{array}{l}\text { MZ N = } 60 \\
\text { First Second }\end{array}$}} & \multirow{2}{*}{\multicolumn{2}{|c|}{$\begin{array}{l}\mathrm{DZ} N=70 \\
\text { First Second }\end{array}$}} \\
\hline & & First & Second & & & & \\
\hline \multirow{2}{*}{$\begin{array}{l}\text { Birth Wt } \\
(\mathrm{kg})\end{array}$} & Mean & $2.58^{\mathrm{a}}$ & $2.38^{a}$ & $2.52^{\mathrm{a}}$ & $2.34^{\mathrm{a}}$ & $2.63^{\mathrm{a}}$ & $2.41^{\mathrm{a}}$ \\
\hline & SEM & 0.05 & 0.05 & 0.07 & 0.07 & 0.07 & 0.06 \\
\hline \multirow{2}{*}{$\begin{array}{l}\text { Height } \\
(\mathrm{cm})\end{array}$} & Mean & 168.32 & 168.36 & 167.52 & 167.65 & 169.02 & 168.97 \\
\hline & SEM & 0.86 & 0.87 & 1.32 & 1.26 & 1.27 & 1.20 \\
\hline \multirow{2}{*}{$\begin{array}{l}\text { BMI } \\
\left(\mathrm{kg} / \mathrm{m}^{2}\right)\end{array}$} & Mean & 25.08 & 24.23 & 24.12 & 23.82 & 25.90 & 24.58 \\
\hline & SEM & 0.45 & 0.31 & 0.47 & 0.46 & 0.72 & 0.42 \\
\hline \multirow{2}{*}{$\begin{array}{l}\text { Body fat } \\
(\%)\end{array}$} & Mean & 26.53 & 25.50 & 25.85 & 25.39 & 26.78 & 25.59 \\
\hline & SEM & 0.82 & 0.72 & 1.22 & 1.09 & 1.11 & 0.96 \\
\hline \multirow{2}{*}{$\begin{array}{l}\text { Systolic }^{b} \\
(\mathrm{~mm} \mathrm{Hg})\end{array}$} & Mean & 125.42 & 127.45 & 126.00 & 126.62 & 124.92 & 128.16 \\
\hline & SEM & 1.26 & 1.30 & 1.73 & 1.76 & 1.82 & 1.90 \\
\hline \multirow{2}{*}{$\begin{array}{l}\text { Diastolic }^{b} \\
(\mathrm{~mm} \mathrm{Hg})\end{array}$} & Mean & 78.84 & 79.47 & 78.61 & 78.25 & 79.04 & 80.52 \\
\hline & SEM & 0.82 & 0.83 & 1.14 & 0.98 & 1.17 & 1.29 \\
\hline
\end{tabular}

${ }^{\text {a }} p<0.001$

${ }^{b}$ excluding pairs with one or both twin on antihypertensive treatment ( $3 \mathrm{MZ}$ and 4 DZ pairs).

In both $\mathrm{MZ}$ and $\mathrm{DZ}$ pairs birth weight was around $200 \mathrm{~g}$ lower in the second twin but adult height was almost identical. There was a tendency for BMI, \% fat and waist circumference to be lower and BP to be higher in the second born twin, especially in DZ pairs but the differences were not statistically significant.

\section{S MONITORING THE EDUCATIONAL ACHIEVEMENTS OF TWINS AND HIGHER MULTIPLES FROM ENTRY TO SCHOOL AT AGE 4}

Peter Tymms

Correspondence address: CEM Centre, University of Durham, Mountjoy 4, Stockton Rd., Durham, UK

This paper reports a quantitative study of the attainments, progress and attitudes of a little over 2500 twins and higher multiples aged 4-5 at the start of school and, in decreasing numbers, at two other time points (one year later and at the age of 7). A computer adaptive assessment (the PIPS baseline) was used for the first two assessments. At 7, their vocabulary, non-verbal ability, reading and maths were tested and their attitudes towards school based activities were assessed. Little difference was found between singles and multiples at the three time points although twins were on average slightly behind singles. The differences were $0.2 \mathrm{SD}$ or less. The discrepancy was similar for all cognitive measures, although, contrary to previous research, it was slightly greater for maths than reading. Paradoxically triplets started school slightly ahead of singles. After school entry the progress of twins and higher multiples was in line with that made by singles. The inattentiveness and hyperactivity of twins aged 5, as rated by their teachers, was slightly greater than that of singles. An unanswered question surrounding the education of twins and higher multiples is whether to separate them or not. One driving hypothesis is that if the twins differ greatly in the cognitive domain then the less able twin might make more rapid progress if educated alone. However, there was no indication that the less academic of pairs in the same school was disadvantaged by his or her relatively low achievement level suggesting that no academic advantage would accrue from separating twins even when their ability scores differ markedly. The findings are discussed in relation to the changing population of twins and higher multiples as well as the developing self-concepts of young children and current classroom practices.

\section{P GENETIC INFLUENCES ON PIAGETIAN CONSERVA- TION ABILITY IN SEVEN YEAR OLD TWINS}

\section{G. Caroline M. van Baal and Dorret I. Boomsma.}

Correspondence address: Vrije Universiteit, Department of Biological Psychology, Van der Boechorststraat 1, 1081 BT Amsterdam, The Netherlands, gcm.van.baal@psy.vu.nl.

In addition to continuous growth, cognitive abilities during childhood show clear discontinuous developmental patterns, as indicated by Piaget in the sixties. The transitions between the different developmental stages coincide with growth spurts in brain maturation (e.g. as indexed by electroencephalographic power and coherence). This may suggest that discontinuous cognitive development will be influenced more by genetic than environmental factors and thus will show a high heritability (i.e., the relative importance of genetic influences on interindividual differences). Surprisingly little research has been done to establish heritabilities of indices of discontinuous cognitive development like the Piagetian 
conservation ability, which generally emerges in children around age 7 years. We addressed this question using a twin design. In establishing and testing for the heritability of conservation ability, we specifically included the possiblity of sex differences. Around their 7 th birthday, 192 twin pairs performed a computerized version of Piaget's conservation ability task, which tests volume conservation ability. Children were either classified as conservers or non-conservers. Tetrachoric twin correlations were monozygotic $(\mathrm{MZ})$ males $=.95(N=37)$; dizygotic (DZ) males $=.44(N=41) ; \mathrm{MZ}$ females $=.59(N=42) ; \mathrm{DZ}$ females $=.40(N=34)$; $\mathrm{DZ}$ male-female pairs $=.51(\mathrm{~N}=38)$. Using threshold models in structural equation modelling, we found heritabilities of $94 \%$ (males) and $62 \%$ (females) $\left(\chi^{2}=12.83, d f=12, p=.381\right)$. Sex differences were significant. $\left(\Delta \chi^{2}=4.04, d f=1, p<.05\right)$, possibly reflecting maturational differences These results support Piaget's assertions regarding the biological origins of conservation ability.

\section{F GENETIC AND ENVIRONMENTAL INFLUENCES ON PROBLEM BEHAVIORS IN 5-YEAR-OLD DUTCH TWINS}

\section{CEM van Beijsterveldt, F.Verhulst, D.I. Boomsma \\ Correspondence address: Dept of Biological Psychology, Vrije Universiteit, Van der Boechorstraat 1, 1081 BT Amsterdam, The Netherlands}

Problem behaviors in young children do not form clearcut diagnostic categories, but involve quantitative variations of behavior that most children display to some degree. It is therefore preferable to examine genetic influences on psychopathology assessed as quantitative variation of behavior rather than a present-vs-absent diagnosis. For this purpose standardized ratings of problem behavior filled in by parents may be a good source of information of child psychopathology. In the present study behavior problems were assessed with items from the Devereux Child Behavior (DCB) rating scale (Spivack \& Spotts, 1966) filled in by the parents. The rating yielded 5 problem scales: aggression, distraction, anxiety, physical coordination, and inability to delay. The sample consisted of 3600 5-year-old twin pairs. Structural equation models were used to examine the contribution of genetic and environmental factors on problem behavior. Univariate genetic analyses yielded large additive genetic effects for all problem scales. We found heritabilities ranging from .50 to .80 . Shared environmental effects were only significant for aggression and anxiety and explained a much smaller part of the variance. Test of sex differences showed no significant results. This research was supported by grant 904-57-094 from the Netherlands Organization for Scientific Research (NWO).

\section{INCLUDING QUANTITATIVE TRAIT LOCI IN STATISTICAL MODELS TO STUDY THE INTERPLAY OF MULTIPLE FACTORS AFFECTING COMPLEX TRAITS}

\section{Edwin JCG van den Oord' \& Harold Snieder ${ }^{2,3}$}

Correspondence address: 'Department of Child and Adolescent Studies, Utrecht University, Utrecht, The Netherlands, ${ }^{2}$ Georgia Prevention Institute, Medical College of Georgia, Augusta, GA, USA ${ }^{3}$ Twin Research and Genetic Epidemiology Unit, St.Thomas'Hospital, London, $U K$

The etiology of complex traits may perhaps best be conceptualized by a coherent network of multiple factors that mediate the influence of the genes on the eventual outcome. Existing quantitative association tests are not suitable for disentangling this complex interplay. We therefore propose a model with observed and latent variables that does not impose restrictions on the number of variables or the direction of their causal relations, and provides a general approach for fitting theoretical models to empirical data. The model is very flexible and 1) allows for genetic effects on the means, variances, and relations between variables, 2) can control for stratification effects on all these components, 3) can be fitted in nuclear families of any size, 4) is estimated using an interpretable parameterization, and 5) can incorporate di- and multi-allelic loci, marker haplotypes, multiple loci simultaneously, and parental genotypes. The model can be estimated with the Mx software and a program that generates the proper Mx script on the basis of few simple questions concerning the data structure and parameter matrices. A simulation study showed that the model yielded correct Type I errors, unbiased parameter estimates, and satisfactory power to discriminate between alternative models. An example is also given that illustrates how the model could be applied to real data.

\section{ASSOCIATIONS BETWEEN TRIPLET ETIOLOGY AND CHILD BEHAVIOR PROBLEMS AT SCHOOL AGE}

\section{Suus M.J. van Hekken \& Jacob F. Orlebeke}

Correspondence address: Vrije Universiteit, Department of Biological psychology,

Van der Boechorststraat 1, 1081 BT Amsterdam, The Netherlands.

From 148 triplet sets, born between January 1987 and December 1993, 42 sets originated naturally (spontaneous), 45 by hormonal induction of ovulation (hormones), and 61 by in vitro fertilization (IVF). Birth weight, birth order, gender and maternal age at delivery time were registered shortly after birth. Between the ages of 6 and 11 years (mean: 7.2 years) parents filled out the Child Behavior Check List (CBCL/4-18), measuring Externalizing behavior and Internalizing behavior.

The main question was: Is problem behavior associated with etiological modus of the triplets? Other questions were: Can previous findings in twin and singleton samples with regard to the associations between birth weight, gender and maternal age on the one hand and externalizing behavior on the other, be replicated? Is there a deviating sex-ratio (more girls) in the hormones-group?

Results. Low birth weight appears to be associated with more externalizing behavior. Young mothers $(<28$ years) gave birth to triplets with more externalizing behavior compared to old mothers ( $>34.6$ years). Boys show more externalizing behavior than girls. Though there is no main effect of etiology on problem behavior, this generally found gender difference is absent in the IVF-group. In the hormones-group, significantly more girls than boys are born. The absence of a gender difference in externalizing behavior in the IVF-triplets, is discussed in the context of the (speculative) possibility of a slightly deviating process of $\mathrm{X}$-inactivation and/or genomic imprinting in this group.

\section{$255 \mathrm{P}$}

VULNERABILITY TO DEPRESSIVENESS AMONG
12,063 ADULTS - EFFECT OF LIFE EVENTS,
SOCIAL SUPPORT AND SOMATIC HEALTH

JyrkiVarjonen, Kalle Romanov, Jaakko Kaprio and Markku Koskenvuo Correspondence address: Vehkanii-tyntie 8 F11, 02180 Espoo, Finland. jyrki.varjonen@dlc.fi

The Finnish Twin Cohort consists of all twin pairs of the same sex born in Finland before 1958 and with both co-twins alive in 1967. The 21-item Beck Depression Inventory (BDI) was included in the 1990 questionnaire to assess depressiveness. The sample consisted 12063 Finnish adults aged 33 to 60 years. We have found remarkably strong effects of various psychosocial variables on depressiveness. These effects remained significant in multi-variate models, such that high BDI scores were associated with stressful life events, and poor somatic health of the subject and lack of social support increased the strength of this association. High neuroticism and low self-assurance were also associated with higher mean BDI Scores. The regression model of the total BDI explained $34.9 \%$ of the variability of the BDI score. The most important variables were stressful life events $(18,5 \%)$, neuroticism $(8,6 \%)$, social network $(4,1 \%)$, self-assurance $(0,9 \%)$, and the interaction term of social network and stressful life events. These effects were seen in both men and women, as well as in those classified as healthy and those considered to have some chronic medical condition. We will further examine if the relationship between life-events and depression is changed when examined within pairs, i.e. by adjustment for genetic factors and childhood experiences. This will be first through analyses of discordant pairs by conditional logistic regression techniques to identify explanatory factors accounting for intra-pair differences in depressiveness. Secondly multivariate genetic modelling of $\mathrm{MZ}$ and $\mathrm{DZ}$ twin pair variance-covariance matrices of these data will be undertaken. It is of special interest to estimate the magnitude of genetic and environmental effect on life events and depression, with particular focus on non-shared environmental factors, as these may prove amenable to intervention.

\section{S FETAL GROWTH AND THE INSULIN-LIKE GROWTH FACTOR (IGF) SYSTEM}

\section{Johan Verhaeghe}

Correspondence address: Dept. of OB/GYN, U.Z. Leuven, Herestraat 49, 3000 Leuven, Belgium

The IGF system includes two ligands (IGF-I and -II), six IGF-binding proteins (IGFBPs), and two IGF receptors (type 1 and 2); it plays a pivotal role in fetal growth regulation. IGF-I and IGF-II are produced by many cell types in virtually all fetal tissues, and stimulate growth $\&$ differentiation in an auto- and paracrine mode, predominantly through the type 1 receptor. The paternally imprinted IGF-II gene is expressed more abundantly than the IGF-I gene. Both homozygous IGF-I gene deletion and paternal IGF-II allele deletion in mice result in intra-uterine growth retardation (IUGR), and their effects are additive. In human fetuses, IGF-I and IGF-II levels are correlated with birth wt, but the correlation between IGF-I and birth wt is more consistent. IGF-I appears 
to be crucial for brain growth, and IGF-II for visceral growth. Univariate analysis in twin fetuses indicates that IGF-I levels are regulated predominantly by genetic factors. IGFBP-1 is probably the most important IGFBP during IU life. IGFBP-1 gene overexpression causes birth wt reduction in some mouse strains and disturbs brain development; hyperinsulinemia and glucose intolerance develop later in life. Conversely, experimental IUGR boosts IGFBP-1 gene expression. In human fetuses, serum IGFBP-1 levels are severalfold higher than in adults and correlate inversely with birth wt. IGF-I, insulin and the PO also correlate negatively with IGFBP-1 levels in the fetus. It is tempting to speculate that chronic hypoxia during IU life upregulates IGFBP-1 gene expression; this IGFBP-1 resetting could later in life result in insulin resistance and glucose intolerance. IGFBP-1 may be a critical factor to explain the thrifty phenotype hypothesis.

\section{S HOW CAN WE PREVENT IATROGENIC MP?}

\section{Hugo C.Verhoeven}

Correspondence address: The Center for Reproductive Medicine. Dusseldorf, Germany

In developed countries, $1 \%$ of babies born result from IVF and other assisted reproductive technologies (ART). Registries indicate a high incidence of multiple pregnancies (MP). In France, the birth frequency of twins after IVF was $23.5 \%$ and of triplets and more $3.75 \%$. Because of new techniques in ART (not all involving IVF), the MP rate is exploding worldwide. The major risks in MP include: very high rates of preterm delivery, low birth weight, intrauterine growth retardation and neonatal and infant mortality. The life of couples who experience a high order MP is changed dramatically for many years to come. A reduction of the proportion of multiple pregnancies should be a major goal for all centers performing ovulation induction with or without IVF. Factors influencing MP are: the number of embryos transferred, the age of the mother, the ovarian stimulation regimen, the embryo quality and the rank of attempt. To reduce the incidence of high order MP, the following should be considered : natural cycle IVF, low dose stimulation regimen, elective transfer of only two or even one embryo, embryo quality, fertilization rate, transfer of blastocysts, cryopreservation, embryo reduction and skill/responsibility. In our experience, reduction of the numbe of embryos transferred to two or one and transfer of blastocysts are the major tools. The transfer of only two embryos does not change the pregnancy (nor twinning) rate but avoids Higher MP. Embryo reduction however, is just an emergency solution.

Conclusion. Can we avoid higher MP? Answer - no. Can we reduce the incidence? Answer — clearly yes.

\section{P GENDER DIFFERENCES IN GENETIC AND ENVIRONMENTAL EFFECTS ON AGGRESSION}

Elina Vierikko, Lea Pulkkinen, Jaakko Kaprio, Richard J. Rose \& Richard J.Viken Correspondence address: University of Jyväskylä, Department of Psychology, P.O. Box 35, FIN-40351 Jyväskylä, Finland vierikko@psyka.jyu.fi

The aim of the present study was to determine whether the same set of genes and environmental effects determine teacher and parental rated children's aggression in males and females, and whether the magnitude of these effects is similar across genders. This study was part of an ongoing nation-wide twin-family study of behavioral development and health habits (Pulkkinen, Kaprio, \& Rose, 1999). The sample consisted of 1647 twin pairs (264 monozygotic male, 300 monozygotic female, 292 dizygotic male, 278 dizygotic female and 513 dizygotic opposite-sex twin pairs), and it represented subsets of five 12-year-old twin cohorts (b. 1983-1987) from Finland. The data were collected with teacher and parental rating forms of the Multidimensional Peer Nomination Inventory (MPNI; Pulkkinen et al., 1999). Gender differences in genetic and environmental influences on aggression were assessed by structural equation models of sex-limitation using MX (Neale, Boker, Xie, \& Maes, 1999). Results suggested significant genetic, shared environmental and specific environmental effects on children's aggression in both males and females, but the best fitting model differed depending on the informant. In parental ratings, the best fitting model suggested same genetic and environmental effects on aggression in both genders, but in teacher ratings, the magnitude of genetic and environmental effects differed across genders males showing lower levels of heritability and higher levels of shared environment than females.

\section{S}

\section{PATTERNS OF FAMILY TRANSMISSION IN PERSONALITY TRAITS: THE PSYCHOPATHIC DEVIATE (Pd) AND THE WIGGINS FEMININITY} (FEM) SCALES

Richard J.Viken, Richard J. Rose, Jaakko Kaprio, \& Markku Koskenvuo.

Correspondence address: Department of Psychology, Indiana University, Bloomington, IN 47405. viken@indiana.edu

When augmented with data on parents, twin samples allow differentiation of genetic and cultural transmission as explanations for parent-child similarity. Thus far, analyses of personality and other individual differences measures have found little evidence for cultural transmission, much to the surprise and disappointment of psychologists (and parents). But it can be argued that temperamental or personality characteristics are supposed to reflect individual differences in basic biological characteristics, and are poor candidates for observing cultural transmission. Traits assumed to be under strong social influence still might show environmental transmission from parents to offspring. We investigated family transmission in two such variables, using 2,500 sets of adolescent twins and their parents drawn from the Finntwin16 project. The Pd scale from the MMPI assesses antisocial characteristics and social deviance. The FEM scale, also derived from the MMPI, assesses endorsement of traditional masculine versus feminine interests, attitudes, and activities. Both variables show significant genetic influences in twin data, and both show significant parent-offspring correlations, although the pattern of associations differs for the two traits. We fit a variety of family transmission models to each variable. Results focus on similarities and differences in the pattern of transmission in the two variables. (Support: NIAAA 08315 and the Academy of Finland 44069)

\section{P TAMBA SURVEY OF WOMEN'S EXPERIENCES OF A MULTIPLE BIRTH IN THE NHS}

JillWalton

Correspondence address: Hon. Medical and Social Research Consultant, Tamba, Picketts, Picketts Lane, Salfords, Surrey RH1 5RG

The present quality agenda in the NHS places more emphasis than ever before on the views of patients and users of health services. Women giving birth to multiples are a minority group and it is therefore important for Tamba as the only voluntary user organisation to canvas the views of women delivering twins or triplets in NHS maternity units. In 1998, we sent out a 4-page questionnaire to individual Tamba members and to over 200 Twin Clubs, to be completed by all women who had given birth to multiples in 1997 . We received responses from 563 families equivalent to $5.5 \%$ of all twins and $12.5 \%$ of all triplets born in the UK in 1997 - whose experiences covered 214 different hospital units. The key findings of the survey are presented, covering quantitative and qualitative information on many aspects of antenatal care, delivery and postnatal care including breastfeeding. General satisfaction levels were high, particularly with care received in neonatal intensive care units. Specific information and antenatal classes for parents were still not readily available. The results of the survey have been used to inform parents and midwives through publications and study days, to update Tamba's own information booklets, and to support new grant applications. We hope to use the findings to set standards for a new Tamba Multiple Birth Excellence Award scheme for NHS maternity units.

\section{F THE MUMBAI PROSPECTIVE TWIN STUDY}

\section{Vandana Walvekar, Pratima Anjaria,Vanita Raut}

Correspondence address: Nowrosjee Wadia Maternity Hospital, Acharya Donde Marg, Parel, Mumbai, Maharastra State, India

Background. The Mumbai Prospective Twin Study group was constituted from the four major teaching hospitals of the city, namely the Wadia Maternity Hospital, the B.Y.L. Nair Hospital, the K.E.M. Hospital, and the L.T. Municipal General Hospital. Its purpose was to study, among other things, chorionicity and outcomes in this hospital-based population.

Methods: A database was constructed using a pickoff sheet specially designed by Dr. Isaac Blickstein of Israel. One person was assigned to collect data on every twin pregnancy at each hospital.

Results. Among the first 146 cases, mean maternal age was 26 years, with 90 percent of mothers being 30 years of age or less, and 91 percent of patients para 2 or less, and 91.8 percent without a family history of twins, and 92.5 percent being spontaneous conceptions. Among the maternal complications were anemia (HB less than 10 gram percent in 36 percent of cases) and antenatal hypertension in 25.4 percent of cases. Of these, 59.4 percent were mild PIH, 21.6 moderate PIH, 10.8 percent severe $\mathrm{PIH}$, and 8.2 percent eclampsia. Preterm labor occurred in 45.2 percent of cases. Birth weight differences after delivery were $<15 \%$ in 63.7 percent, $15-25 \%$ in 19.9 percent, and $>25 \%$ in in 16.4 percent. Of the total 292 births, there were 11 stillbirths and 17 neonatal deaths. 
The perinatal mortality rate was 98 per thousand total births and the neonatal death rate was 62 per thousand live births.

Conclusion. This is the first attempt to systematically study maternal and fetal outcomes in an Indian population of twins. The work is ongoing.

\section{S CHARACTERISATION OF ANASTOMOTIC ANATOMY IN TWIN-TWIN TRANSFUSION SYNDROME.}

\section{Ling Wee, Myles Taylor, Nicholas Fisk}

Correspondence address: Centre for Fetal Care, Queen Charlotte's and Chelsea Hospital, Du Cane Road, Hammersmith, London, W12 ONN

Aim: To characterise the macroscopic appearances of arterio-venous anastomoses (AVAs) in monochorionic (MC) twins with and without twin-twin transfusion syndrome.

Main Outcome Measures. To determine in TTTS pregnancies and uncomplicated MC pregnancies: 1) number of and direction of AVAs 2) diameter of AVAs, 3) AVA placental location 4) AVA intra arteriovenous angle and 5) AVA branching order

Results. Preliminary results from $50 \mathrm{MC}$ placentae showed that there was no difference in the overall number of AVA (median 2, range 2-7 versus 4 , range $0-11$ ), diameter of AVA (mean $=1.5 \mathrm{~mm}$ versus $1.4 \mathrm{~mm}$ ) and their angulation (mean 130 degrees, range 100-150 versus 125 degrees, range 110-180) between the TTTS group and uncomplicated MC pregnancies Net anastomotic gradient was also not associated with TTTS development. AVAs in either direction were nearer to the uppermost or lowermos edge of placenta in the TTTS group compared to the control MC group. The branching order of both arteries and veins for AVAs were not significantly different across the groups for vessels from larger to smaller twin. However with AVA travelling from smaller to larger twin, there was a lower branch order in the artery in the TTTS and growth discordance groups compared to uncomplicated MC pregnancies

Conclusion. Subtle differences have been observed in AVA anastomotic configuration in placentae affected by TTTS and these may further contribute to understanding of this disease

\section{P MULTI-FETAL PREGNANCY:} ACHIEVING SUCCESSFUL OUTCOMES

\section{KellyWillenberg and Patti Beemer}

Correspondence address: Correspondence address: P.O. Box 438, Thompson Station,

Tennessee, 37179-0438, USA

Balancing the joy and anticipation of bringing higher-order multiple birth children into the world with the very real, practical concern of managing a high-risk pregnancy is a challenge for all involved. Through extensive research, the National Organization of Mothers of Twins Clubs, Inc. has prepared a "hands-on" program for physicians, support personnel and expectant parents of triplets, quadruplets and more. These materials focusing on ways to improve communication and outcomes will be shared during this session. Issues covered during this presentation include pregnancy management, tests and procedures, nutrition, medical intervention, complications, stress management and assistance with transition needs after the birth of the babies. Resources to facilitate communication between physicians and patients have been successfully researched and developed in a variety of formats to aid in their use and effectiveness. The continuum of advancing health care will be examined and tools to assist the provider, administrator and parents in making the most well informed choices will be distributed. Understanding the unique experiences and issues facing mothers with multi-fetal pregnancies and coordinating the best techniques for their medica and psychological care is crucial to creating the most successful outcome for families with triplets, quadruplets and quintuplets.

\section{P SOCIAL ALIENATION IN ADOLESCENT FINNISH TWINS}

Torsten Winter, Jaakko Kaprio, Richard J.Viken, Richard J. Rose.

Correspondence address: Department of Public Health, University of Helsinki. Box 41,

Mannerheimintie 172, Helsinki, Finland Torsten.Winter@helsinki.fi

Social Alienation has an important effect on many kinds of behaviour, including, for example, adolescent drinking. Despite its importance, there have been few genetic studies of the phenomenon. We measured Social Alienation with a Harris-Lingoes scale, consisting of nine items from the Minnesota Multiphasic Personality Inventory, in a population-based family data set consisting of parents and their 16-year-old co-twins in 1,882 families (FinnTwin16). According to a previous study, Social Alienation, measured with this scale, was strongly related to some social environments (unemployment among adults), but not to other kinds of environmental variation (regions). Our intention in this study was to broaden our knowledge of social alienation by using a twin-family research design. The relationship of social alienation between the co-twins and their parents was studied using correlations. We also modelled the relationships among the family members using twin-family models in Mx.

Results. Mean scores and standard deviation: Boys 2.46 (1.97), Girls 2.76 (1.98), Fathers 1.97 (1.89), Mothers 1.84 (1.77). Correlations among family members: father-mother 0.21 , father-son 0.09 , father-daughter 0.12 , mother-son 0.10 , mother-daughter 0.12 . Correlations among twins: Female MZ 0.52, Male MZ 0.50, Female DZ 0.32, Male DZ 0.29, Opp.sex 0.11 .

Conclusion. The pattern of family correlation support familial aggregation and possible genetic influences on social alienation.

\section{P NO HERITABILITY OF PARKINSONISM IN SWEDISH TWINS}

\section{Karin Wirdefeldt, Nancy L Pedersen}

Correspondence address: Department of Medical Epidemiology, Karolinska Institutet, Stockholm, Sweden

One large twin study using the all male NAS/NRC World War II Veteran Twin Registry reported similar concordance rates for Parkinson's disease in monozyg otic and dizygotic twins. We evaluated concordances in the Swedish Twin Registry, where both sexes and opposite sexed pairs are included. A total of 25,064 twins in 8,597 pairs above 55 years were screened for most complex diseases using computer assisted telephone interviews. The twins were asked whether they have received a Parkinson's diagnosis, take anti-Parkins on medication, and whether they experience a variety of parkinsonian symptoms. The symptoms were difficulty to stand up, smaller letters in handwriting, balance problems, feet stuck on the floor when walking, dragging feet when walking, stiffness of the face, shaky arms or legs, difficulty buttoning buttons and low voice. Validation of screening questions is ongoing using medical records and somatic examinations. Probandwise concordance rates were calculated stratified by zygosity, sex and age, considering those with positive answers to the questions about diagnosis or treatment and those with three positive answers to the symptom questions as affected. Concordances were $19.8 \%$ for monozygotic twins $(19.5 \%$ for men and $20.0 \%$ for women), $20.1 \%$ for dizygotic twins (20.1\% for both men and women) and $11.5 \%$ for opposite sexed twins. Monozygotic concordances were slightly greater than dizygotic among twins age 55-64, suggesting a potential influence of genetic effects for early onset cases.

\section{P FAVORITISM IN MOTHERS OF MULTIPLE BIRTH CHILDREN}

Yoshie Yokoyama

Correspondence address: College of Medical Technology, Kyoto University, 53 Kawaharacho, Shogoin, Sakyo-ku, Kyoto, 606-8507 Japan

The birth of twins is associated with an increased risk of child abuse. In Japan, a nationwide survey found that $10 \%$ of child abuse victims were products of multiple births, almost ten times the rate found in the general population. In particular, in multiple births, the risk of abuse of only one child is high, and the abusers are mostly the mothers. Moreover, favoritism by parents has been suggested as the reason for this tendency. This study investigated the state of occurrence of favoritism in mothers with multiple birth children and identified factors associated with increased risk. The 231 subjects were 126 mothers of twins, 96 of triplets, 7 of quadruplets and 2 of quintuplets. The state of health in the mothers who showed a tendency towards favoritism was significantly poorer $(p<0.05)$ than in those who did not show a tendency towards favoritism. The rate of those who frequently contracted upper respiratory infections was also significantly higher $(p<0.01)$ in the mothers who showed a tendency towards favoritism. The state of fatigue based on characteristics in the Fatigue Symptom Index and 5-grade ratings of physical and mental fatigue was significantly more advanced in the mothers who showed a tendency towards favoritism than in those who did not show a tendency towards favoritism. The state of sleep was poorer in the mothers who showed a tendency towards favoritism. 